Comment. Math. Helv. 76 (2001) 263-299

(C) 2001 Birkhäuser Verlag, Basel

Commentarii Mathematici Helvetici

\title{
On orbit closures of spherical subgroups in flag varieties
}

\author{
Michel Brion
}

\begin{abstract}
Let $\mathcal{F}$ be the flag variety of a complex semi-simple group $\mathrm{G}$, let $\mathrm{H}$ be an algebraic subgroup of $\mathrm{G}$ acting on $\mathcal{F}$ with finitely many orbits, and let $\mathrm{V}$ be an $\mathrm{H}$-orbit closure in $\mathcal{F}$. Expanding the cohomology class of $\mathrm{V}$ in the basis of Schubert classes defines a union $\mathrm{V}_{0}$ of Schubert varieties in $\mathcal{F}$ with positive multiplicities. If $G$ is simply-laced, we show that these multiplicities are equal to the same power of 2 . For arbitrary $G$, we show that $V_{0}$ is connected in codimension 1. If moreover all multiplicities are 1 , we show that the singularities of $\mathrm{V}$ are rational and we construct a flat degeneration of $\mathrm{V}$ to $\mathrm{V}_{0}$ in $\mathcal{F}$. Thus, for any effective line bundle $\mathrm{L}$ on $\mathcal{F}$, the restriction map $H^{0}(\mathcal{F}, \mathrm{L}) \rightarrow H^{0}(\mathrm{~V}, \mathrm{~L})$ is surjective, and $H^{n}(\mathrm{~V}, \mathrm{~L})=0$ for all $n \geq 1$.
\end{abstract}

Mathematics Subject Classification (2000). 14M15, 14L30, 14B05, 14 D05.

Keywords. Flag varieties, Schubert varieties, spherical homogeneous spaces, rational singularities.

\section{Introduction}

Let $\mathrm{G}$ be a connected complex reductive group, $\mathrm{B}$ a Borel subgroup and $\mathrm{H}$ a spherical subgroup, that is, $\mathrm{H}$ is an algebraic subgroup having only finitely many orbits in the flag variety G/B. The classification of these orbits and the geometry of their closures are of importance in representation theory. Equivalent questions concern the $\mathrm{B}$-orbits in $\mathrm{G} / \mathrm{H}$ and their closures in that space. More generally, one may consider $\mathrm{B}$-orbit closures in an equivariant embedding $\mathrm{X}$ of $\mathrm{G} / \mathrm{H}$; these play an essential role in the geometry and topology of $\mathrm{X}$.

The set $\mathcal{B}(\mathrm{G} / \mathrm{H})$ of $\mathrm{B}$-orbit closures in $\mathrm{G} / \mathrm{H}$ is partially ordered by inclusion. A weaker order $\preceq$ on $\mathcal{B}(\mathrm{G} / \mathrm{H})$ is defined by: $\mathrm{Y} \preceq \mathrm{Y}^{\prime}$ if there exists a sequence $\left(\mathrm{P}_{1}, \ldots, \mathrm{P}_{n}\right)$ of parabolic subgroups containing $\mathrm{B}$ such that $\mathrm{Y}^{\prime}=\mathrm{P}_{1} \cdots \mathrm{P}_{n} \mathrm{Y}$. In this paper, we investigate this weak order and its associated graph, with applications to B-orbit closures in "regular" completions of $\mathrm{G} / \mathrm{H}$, and in turn to the geometry of $\mathrm{H}$-orbit closures in $\mathrm{G} / \mathrm{B}$.

The case where $\mathrm{H}$ equals $\mathrm{B}$ is well known: then $\mathcal{B}(\mathrm{G} / \mathrm{H})$ identifies to the Weyl group $\mathrm{W}$, and the inclusion (resp. weak) order is the Bruhat-Chevalley (resp. left weak) order, see e.g. [13] 5.9. The $\mathrm{H}$-orbit closures in G/B are the Schubert vari- 
eties; using an explicit desingularization, reduction to positive characteristics and Frobenius splitting, one shows that their singularities are rational, in particular, they are normal and Cohen-Macaulay ([19]).

Another important class of spherical subgroups consists of symmetric subgroups, that is, fixed points of involutions of G. Orbit closures of symmetric subgroups in flag varieties admit explicit resolutions of singularities as well, and their inclusion and weak orders have been studied in detail by Richardson and Springer ([20], [21], [23]). But their singularities are far from being fully understood; non-normal examples were constructed by Barbasch and Evens ([1]).

Returning to a spherical subgroup $\mathrm{H}$, classical projective geometry yields many examples of $\mathrm{H}$-orbit closures in flag varieties that are neither normal nor CohenMacaulay, e.g., the variety of all secant lines to a given conic in projective $n$ space where $n \geq 3$ (see Example 6 for details). A criterion for having rational singularities will be formulated below, in terms of the oriented graph $\Gamma(\mathrm{G} / \mathrm{H})$ associated with the weak order.

For this, we endow that graph with additional data, as in [20]: each edge from $\mathrm{Y}$ to $\mathrm{Y}^{\prime}$ is labeled by a simple root of $\mathrm{G}$ corresponding to a minimal parabolic subgroup $\mathrm{P}$ such that $\mathrm{PY}=\mathrm{Y}^{\prime}$. The degree of the associated morphism $\mathrm{P} \times{ }^{\mathrm{B}} \mathrm{Y} \rightarrow \mathrm{Y}^{\prime}$ being 1 or 2 , this defines simple and double edges. There may be several edges with the same endpoints, but they are simultaneously simple or double (Proposition 1).

In the symmetric case, a key result is that all minimal elements of $\Gamma(\mathrm{G} / \mathrm{H})$ are closed orbits ([20]); thus, one may study orbit closures by going up along oriented paths in the graph. This does not extend to the general case (see e.g. Example 2 below), but it is easy to see that the unique maximal element is $G / H$. This enables an inductive study of orbit closures by going down along oriented paths; most of our results are obtained in this way.

For instance, $\Gamma(\mathrm{G} / \mathrm{H})$ determines the cohomology class of any $\mathrm{H}$-orbit closure $\mathrm{V}$ in $\mathrm{G} / \mathrm{B}$, that is, its intersection numbers with Schubert varieties, $\int_{\mathrm{G} / \mathrm{B}}[\mathrm{V}]$. $[\overline{\mathrm{B} w \mathrm{~B} / \mathrm{B}}]=d(\mathrm{~V}, w)$, for all $w \in \mathrm{W}$. Consider indeed the associated $\mathrm{B}$-orbit closure $\mathrm{Y}$ in $\mathrm{G} / \mathrm{H}$. Join it to $\mathrm{G} / \mathrm{H}$ by an oriented path $\gamma$; denote by $\mathrm{D}(\gamma)$ its number of double edges, and by $w(\gamma)$ the product in $\mathrm{W}$ of the simple reflections associated with its labels. Then $d(\mathrm{~V}, w(\gamma))=2^{\mathrm{D}(\gamma)}$ (in particular, $\mathrm{D}(\gamma)$ depends only on $\mathrm{V}$ and $w(\gamma))$ and, moreover, all non-zero intersection numbers $d(\mathrm{~V}, w)$ are obtained in this way ([6]). Equivalently, we have in the cohomology ring of G/B :

$$
[\mathrm{V}]=\sum_{w=w(\gamma)} 2^{\mathrm{D}(\gamma)}\left[\overline{\mathrm{B} w_{0} w \mathrm{~B}} / \mathrm{B}\right]
$$

where $w_{0}$ denotes the longest element of $\mathrm{W}$.

The oriented paths in $\Gamma(\mathrm{G} / \mathrm{H})$ and their associated Weyl group elements are studied in Section 1. The main tool is a notion of neighbor paths that reduces several questions to the case where $G$ has rank two. Using this, we show that the 
union of Schubert varieties

$$
\mathrm{V}_{0}=\bigcup_{w=w(\gamma)} \overline{\mathrm{B} w_{0} w \mathrm{~B}} / \mathrm{B}
$$

is connected in codimension 1 (Corollary 7). If moreover $\mathrm{G}$ is simply-laced, then the number of double edges in any oriented path depends only on its endpoints (Proposition 5). As a consequence, all non-zero coefficients of [V] in the basis of Schubert cycles are equal. For symmetric spaces, this can also be deduced from a combinatorial result of Richardson and Springer [24]. It does not extend to multiply-laced groups, see Example 4 in Section 1.

In Section 2, we analyze the intersections of $\mathrm{B}$-orbit closures with $\mathrm{G}$-orbit closures in an equivariant completion $\mathrm{X}$ of $\mathrm{G} / \mathrm{H}$ that is regular in the sense of Bifet, De Concini and Procesi [2]; these intersections are proper, by [6]. The main ingredient is a "slice" $\mathrm{S}_{\mathrm{Y}, \gamma}$, a toric variety associated with a B -orbit closure $\mathrm{Y}$ meeting $\mathrm{G} / \mathrm{H}$ and with an oriented path $\gamma$ from $\mathrm{Y}$ to $\mathrm{G} / \mathrm{H}$. Given a $\mathrm{G}$-orbit closure $\mathrm{X}^{\prime}$ and an irreducible component $\mathrm{Y}^{\prime}$ of $\mathrm{Y} \cap \mathrm{X}^{\prime}$, there exists a "slice" meeting $\mathrm{Y}^{\prime}$, and the corresponding intersection multiplicity divides $2^{\mathrm{D}(\gamma)}$, with equality if $\mathrm{X}^{\prime}$ is a closed $\mathrm{G}$-orbit (Theorem 1).

This distinguishes the $\mathrm{B}$-orbit closures $\mathrm{Y}$ such that all oriented paths in $\Gamma(\mathrm{G} / \mathrm{H})$ with source $\mathrm{Y}$ contain only simple edges; we call them multiplicity-free. In a regular completion of $\mathrm{G} / \mathrm{H}$, any irreducible component of the intersection of multiplicity-free $\mathrm{Y}$ with a $\mathrm{G}$-orbit closure is multiplicity-free as well, and the corresponding intersection multiplicity is 1 (Corollary 3 ).

In Section 3, we relate our approach to work of Knop ([17], [18]). He defined an action of $\mathrm{W}$ on the set $\mathcal{B}(\mathrm{G} / \mathrm{H})$ such that the $\mathrm{W}$-conjugates of $\mathrm{G} / \mathrm{H}$ are the orbit closures of maximal rank (in the sense of [18].) Moreover, the isotropy group $\mathrm{W}_{(\mathrm{G} / \mathrm{H})}$ is closely related to the "Weyl group of $\mathrm{G} / \mathrm{H}$ " studied in [17], a generalization of the little Weyl group of symmetric spaces.

It is easy to see that all orbit closures of maximal rank are multiplicity-free. We describe their intersections with $\mathrm{G}$-orbit closures in a regular completion, in terms of $\mathrm{W}$ and $\mathrm{W}_{(\mathrm{G} / \mathrm{H})}$ (Proposition 9). As applications, we show that all elements of $\mathrm{W}$ of minimal length in a given $\mathrm{W}_{(\mathrm{G} / \mathrm{H})}$-coset have the same length, and that $\mathrm{W}_{(\mathrm{G} / \mathrm{H})}$ is generated by reflections or products of two commuting reflections of $\mathrm{W}$. This in turn yields another proof of the fact that the Weyl group of $\mathrm{G} / \mathrm{H}$ is generated by reflections, a result of Knop [17].

Section 4 contains our main result, Theorem 5: the singularities of any multiplicity-free $\mathrm{B}$-orbit closure $\mathrm{Y}$ in a spherical variety $\mathrm{X}$ of $\mathrm{G} / \mathrm{H}$ are rational. If moreover $\mathrm{X}$ is regular, then the scheme-theoretical intersection of $\mathrm{Y}$ with any $\mathrm{G}$-orbit closure is reduced. Since no explicit desingularization of $\mathrm{Y}$ is known in this generality, the proof is indirect; it goes by decreasing induction on dimension like Seshadri's proof of normality of Schubert varieties [22].

For a $\mathrm{H}$-orbit closure $\mathrm{V}$ in $\mathrm{G} / \mathrm{B}$, the corresponding $\mathrm{B}$-orbit closure $\mathrm{Y}$ is multiplicity-free if and only if $[\mathrm{V}]=\left[\mathrm{V}_{0}\right]$. In that case, we construct a flat degeneration of $\mathrm{V}$ to $\mathrm{V}_{0}$ where the latter is viewed as a reduced subscheme of 
G/B (Corollary 6). Thus, the equality $[\mathrm{V}]=\left[\mathrm{V}_{0}\right]$ holds in the Grothendieck group of $\mathrm{G} / \mathrm{B}$ as well. Moreover, for any effective line bundle $\mathrm{L}$ on $\mathrm{G} / \mathrm{B}$, the cohomology groups $H^{n}(\mathrm{~V}, \mathrm{~L})$ vanish for $n \geq 1$, and the restriction map $H^{0}(\mathrm{G} / \mathrm{B}, \mathrm{L}) \rightarrow H^{0}(\mathrm{~V}, \mathrm{~L})$ is surjective (Corollary 8$)$.

This yields a more concrete realization of the $\mathrm{H}$-module $H^{0}(\mathrm{~V}, \mathrm{~L})$ : choose the Borel subgroup $\mathrm{B}$ such that $\mathrm{V}$ is the closure of $\mathrm{HB} / \mathrm{B}$ and let $\lambda$ be the dominant weight of $\mathrm{B}$ associated with $\mathrm{L}$. Then $H^{0}(\mathrm{G} / \mathrm{B}, \mathrm{L})$ is the dual of the simple G-module $\mathrm{V}(\lambda)$ with highest weight $\lambda$, and $H^{0}(\mathrm{~V}, \mathrm{~L})$ is the dual of the $\mathrm{H}$ submodule of $\mathrm{V}(\lambda)$ generated by the highest weight line $\ell_{\lambda}$. As $\mathrm{V}$ degenerates to $\mathrm{V}_{0}$, this $\mathrm{H}$-module degenerates to a $\mathrm{B}$-module of the same dimension, generated by the extremal weight lines $\ell_{w_{0} w \lambda}$ for all $w$ as above. This construction will be developed elsewhere.

When applied to symmetric spaces and combined with Theorem B of [1], this surjectivity result implies a version of the PRV conjecture, see [1] §6. It holds for another class of (smooth) $\mathrm{H}$-orbit closures as well, but not to all of them, see [4]. In fact, surjectivity of the restriction map for all effective L's and all $\mathrm{H}$-orbit closures is equivalent to their multiplicity-freeness (Proposition 10.)

A remarkable example of a spherical homogeneous space where all orbit closures of a Borel subgroup have maximal rank (and hence are multiplicity-free) is the group $G$ viewed as a homogeneous space under $G \times G$. If moreover $G$ is adjoint, then it has a canonical regular completion $\mathrm{X}$. It is proved in [8] that the $\mathrm{B} \times \mathrm{B}$ orbit closures in $\mathrm{X}$ are normal and that their intersections are reduced. This follows from the fact that $\mathrm{X}$ is Frobenius split compatibly with all of these closures. It would be tempting to generalize this to any spherical variety $\mathrm{X}$. By [5], $\mathrm{X}$ is Frobenius split compatibly with all $\mathrm{G}$-orbit closures; but this does not extend to $\mathrm{B}$-orbit closures, since their intersections may be not reduced. This happens, e.g., for the space of all symmetric $n \times n$ matrices of determinant 1 , that is, the symmetric space $\mathrm{SL}(n) / \mathrm{SO}(n)$ : consider the subvarieties $\left(a_{11}=0\right)$ and $\left(a_{11} a_{22}-a_{12}^{2}=0\right)$.

So the present paper generalizes results of [8] to all regular completions of spherical homogeneous spaces, by other methods. It raises many further questions, e.g., is it true that the normalization of any B-orbit closure in a spherical variety has rational singularities? And do our results extend to positive characteristics?

\section{Notation}

Let $\mathrm{G}$ be a complex connected reductive algebraic group. Let $\mathrm{B}, \mathrm{B}^{-}$be opposite Borel subgroups of $\mathrm{G}$, with unipotent radicals $\mathrm{U}, \mathrm{U}^{-}$and common torus $\mathrm{T}$, a maximal torus of $\mathrm{G}$. Let $X$ be the character group of B; we identify $X$ with the character group of $\mathrm{T}$ and we choose a scalar product on $X$, invariant under the Weyl group W . Let $\Phi$ be the root system of $(\mathrm{G}, \mathrm{T})$, with the subset $\Phi^{+}$of positive roots, i.e., of roots of $(\mathrm{B}, \mathrm{T})$, and its subset $\Delta$ of simple roots. 
For $\alpha \in \Delta$, let $s_{\alpha} \in \mathrm{W}$ be the corresponding simple reflection, and let $\mathrm{P}_{\alpha}=\mathrm{B} \cup \mathrm{B} s_{\alpha} \mathrm{B}$ be the corresponding minimal parabolic subgroup. For any subset $\mathrm{I}$ of $\Delta$, let $\mathrm{P}_{\mathrm{I}}$ be the subgroup of $\mathrm{G}$ generated by the $\mathrm{P}_{\alpha}, \alpha \in \mathrm{I}$. The map $\mathrm{I} \rightarrow \mathrm{P}_{\mathrm{I}}$ is a bijection from subsets of $\Delta$ to subgroups of $\mathrm{G}$ containing $\mathrm{B}$, that is, to standard parabolic subgroups of $G$.

Let $\mathrm{L}_{\mathrm{I}}$ be the Levi subgroup of $\mathrm{P}_{\mathrm{I}}$ that contains $\mathrm{T}$; let $\Phi_{\mathrm{I}}$ be the root system of $\left(\mathrm{L}_{\mathrm{I}}, \mathrm{T}\right)$, with Weyl group $\mathrm{W}_{\mathrm{I}}$. We denote by $\ell$ the length function on $\mathrm{W}$ and by $\mathrm{W}^{\mathrm{I}}$ the set of all $w \in \mathrm{W}$ such that $\ell\left(w s_{\alpha}\right)=\ell(w)+1$ for all $\alpha \in \mathrm{I}$ (this amounts to: $\left.w(\mathrm{I}) \subseteq \Phi^{+}\right)$. Then $\mathrm{W}^{\mathrm{I}}$ is a system of representatives of the set of right cosets $\mathrm{W} / \mathrm{W}_{\mathrm{I}}$.

\section{The weak order and its graph}

In what follows, we denote by $\mathrm{G} / \mathrm{H}$ a spherical homogeneous space and by $\mathcal{B}(\mathrm{G} / \mathrm{H})$ the set of $B$-orbit closures in $G / H$. One associates to a given $Y \in \mathcal{B}(G / H)$ several combinatorial invariants, see [18]: The character group $X(Y)$ is the set of all characters of $\mathrm{B}$ that arise as weights of eigenvectors of $\mathrm{B}$ in the function field $\mathbb{C}(\mathrm{Y})$. Then $X(\mathrm{Y})$ is a free abelian group of finite rank $r(\mathrm{Y})$, the rank of $\mathrm{Y}$.

Let $\mathrm{Y}^{0}$ be the open $\mathrm{B}$-orbit in $\mathrm{Y}$ and let $\mathrm{P}(\mathrm{Y})$ be the set of all $g \in \mathrm{G}$ such that $g \mathrm{Y}^{0}=\mathrm{Y}^{0}$; then $\mathrm{P}(\mathrm{Y})$ is a standard parabolic subgroup of $\mathrm{G}$. Let $\mathrm{L}(\mathrm{Y})$ be its Levi subgroup that contains $\mathrm{T}$ and let $\Delta(\mathrm{Y})$ be the corresponding subset of $\Delta$ : the set of simple roots of $\mathrm{Y}$.

We note some easy properties of these invariants.

Lemma 1. (i) $X(\mathrm{Y})$ is isomorphic to the quotient of the group of invertible regular functions on $\mathrm{Y}^{0}$, by the subgroup of constant non-zero functions.

(ii) The derived subgroup $[\mathrm{L}(\mathrm{Y}), \mathrm{L}(\mathrm{Y})]$ fixes a point of $\mathrm{Y}^{0}$.

(iii) The group $\mathrm{W}_{\Delta(\mathrm{Y})}$ fixes pointwise $X(\mathrm{Y})$. Equivalently, any simple root of $\mathrm{Y}$ is orthogonal to $X(\mathrm{Y})$.

Proof. (i) Let $f$ be an eigenvector of $\mathrm{B}$ in $\mathbb{C}(\mathrm{Y})$ with weight $\chi(f)$. Then $f$ restricts to an invertible regular function on $\mathrm{Y}^{0}$, and is uniquely determined by $\chi(f)$ up to a constant. Conversely, let $f$ be an invertible regular function on the $\mathrm{B}$-orbit $\mathrm{Y}^{0}$. Then $f$ pulls back to an invertible regular function on $\mathrm{B}$, that is, to a scalar multiple of a character of $\mathrm{B}$. Thus, $f$ is an eigenvector of $\mathrm{B}$ in $\mathbb{C}(\mathrm{Y})$. (ii) Choose $y \in \mathrm{Y}^{0}$. Let $\mathrm{B}_{y}$ (resp. $\left.\mathrm{P}(\mathrm{Y})_{y}\right)$ be the isotropy group of $y$ in $\mathrm{B}$ (resp. $\mathrm{P}(\mathrm{Y})$ ). Since $\mathrm{Y}^{0}=\mathrm{B}_{y}=\mathrm{P}(\mathrm{Y})_{y}$, we have $\mathrm{P}(\mathrm{Y})=\mathrm{BP}(\mathrm{Y})_{y}$. Thus, $\mathrm{P}(\mathrm{Y})_{y}$ acts transitively on $\mathrm{P}(\mathrm{Y}) / \mathrm{B}$, the flag variety of $\mathrm{P}(\mathrm{Y})$. Using e.g. [9], it follows that $\mathrm{P}(\mathrm{Y})_{y}$ contains a maximal connected semi-simple subgroup of $\mathrm{P}(\mathrm{Y})$, that is, a conjugate of $[\mathrm{L}(\mathrm{Y}), \mathrm{L}(\mathrm{Y})]$.

(iii) follows from [18] Lemma 3.2; it can be deduced from (ii) as well. 
Let $\mathrm{Y}, \mathrm{Y}^{\prime} \in \mathcal{B}(\mathrm{G} / \mathrm{H})$ and let $\alpha \in \Delta$. We say that $\alpha$ (or $\mathrm{P}_{\alpha}$ ) raises $\mathrm{Y}$ to $\mathrm{Y}^{\prime}$ if $\mathrm{Y}^{\prime}=\mathrm{P}_{\alpha} \mathrm{Y} \neq \mathrm{Y}$. Let then

$$
f_{\mathrm{Y}, \alpha}: \mathrm{P}_{\alpha} \times{ }^{\mathrm{B}} \mathrm{Y} \rightarrow \mathrm{P}_{\alpha} / \mathrm{B}
$$

be the homogeneous bundle with fiber the $\mathrm{B}$-variety $\mathrm{Y}$ and basis $\mathrm{P}_{\alpha} / \mathrm{B}$ (isomorphic to projective line.) The map $\mathrm{P}_{\alpha} \times \mathrm{Y} \rightarrow \mathrm{G} / \mathrm{H},(p, y) \mapsto p y$ factors through a proper morphism

$$
\pi_{\mathrm{Y}, \alpha}: \mathrm{P}_{\alpha} \times{ }^{\mathrm{B}} \mathrm{Y} \rightarrow \mathrm{Y}^{\prime}=\mathrm{P}_{\alpha} \mathrm{Y}
$$

that restricts to a finite morphism $\mathrm{P}_{\alpha} \times{ }^{\mathrm{B}} \mathrm{Y}^{0} \rightarrow \mathrm{P}_{\alpha} \mathrm{Y}^{0}$. In particular, $\operatorname{dim}\left(\mathrm{Y}^{\prime}\right)=$ $\operatorname{dim}(\mathrm{Y})+1$.

By [20] or [18] Lemma 3.2, one of the following three cases occurs.

- Type $\mathrm{U}: \mathrm{P}_{\alpha} \mathrm{Y}^{0}=\mathrm{Y}^{\prime 0} \cup \mathrm{Y}^{0}$ and $\pi_{\mathrm{Y}, \alpha}$ is birational. Then $X\left(\mathrm{Y}^{\prime}\right)=s_{\alpha} X(\mathrm{Y})$; thus, $r\left(\mathrm{Y}^{\prime}\right)=r(\mathrm{Y})$.

- Type $\mathrm{T}: \mathrm{P}_{\alpha} \mathrm{Y}^{0}=\mathrm{Y}^{\prime 0} \cup \mathrm{Y}^{0} \cup \mathrm{Y}_{-}^{0}$ for some $\mathrm{Y}_{-} \in \mathcal{B}(\mathrm{G} / \mathrm{H})$ of the same dimension as $\mathrm{Y}$, and $\pi_{\mathrm{Y}, \alpha}$ is birational. Then $r(\mathrm{Y})=r\left(\mathrm{Y}_{-}\right)=r\left(\mathrm{Y}^{\prime}\right)-1$.

- Type $\mathrm{N}: \mathrm{P}_{\alpha} \mathrm{Y}^{0}=\mathrm{Y}^{\prime 0} \cup \mathrm{Y}^{0}$ and $\pi_{\mathrm{Y}, \alpha}$ has degree 2. Then $r(\mathrm{Y})=r\left(\mathrm{Y}^{\prime}\right)-1$. In particular, $r(\mathrm{Y}) \leq r\left(\mathrm{P}_{\alpha} \mathrm{Y}\right)$ with equality if and only if $\alpha$ has type $\mathrm{U}$.

Our notation for types differs from that in [20] and [18]; it can be explained as follows. Choose $y \in \mathrm{Y}^{0}$ with isotropy group $\left(\mathrm{P}_{\alpha}\right)_{y}$ in $\mathrm{P}_{\alpha}$. Then $\left(\mathrm{P}_{\alpha}\right)_{y}$ acts on $\mathrm{P}_{\alpha} / \mathrm{B} \cong \mathbb{P}^{1}$ with finitely many orbits, since $\mathrm{B}$ acts on $\mathrm{P}_{\alpha} \mathrm{Y}^{0} \cong \mathrm{P}_{\alpha} /\left(\mathrm{P}_{\alpha}\right)_{y}$ with finitely many orbits. By [20] or [18], the image of $\left(\mathrm{P}_{\alpha}\right)_{y}$ in $\operatorname{Aut}\left(\mathrm{P}_{\alpha} / \mathrm{B}\right) \cong \mathrm{PGL}(2)$ is a torus (resp. the normalizer of a torus) in type $\mathrm{T}$ (resp. N); in type $\mathrm{U}$, this image contains a non-trivial unipotent subgroup.

Definition. Let $\Gamma(\mathrm{G} / \mathrm{H})$ be the oriented graph with vertices the elements of $\mathcal{B}(\mathrm{G} / \mathrm{H})$ and edges labeled by $\Delta$, where $\mathrm{Y}$ is joined to $\mathrm{Y}^{\prime}$ by an edge of label $\alpha$ if that simple root raises $\mathrm{Y}$ to $\mathrm{Y}^{\prime}$. This edge is simple (resp. double) if $\pi_{\mathrm{Y}, \alpha}$ has degree 1 (resp. 2.) The partial order $\preceq$ on $\mathcal{B}(\mathrm{G} / \mathrm{H})$ with oriented graph $\Gamma(\mathrm{G} / \mathrm{H})$ will be called the weak order.

Note that the dimension and rank functions are compatible with $\preceq$. We shall see that $\mathrm{Y}, \mathrm{Y}^{\prime} \in \mathcal{B}(\mathrm{G} / \mathrm{H})$ satisfy $\mathrm{Y} \preceq \mathrm{Y}^{\prime}$ if and only if $\mathrm{Y}^{\prime}=\overline{\mathrm{B} w \mathrm{Y}}$ for some $w \in \mathrm{W}$ (Corollary 1.)

In the case where $\mathrm{H}$ is a parabolic subgroup of $\mathrm{G}$, the rank function is zero. Thus, all edges are of type $\mathrm{U}$; in particular, they are simple. Here is another example, where double edges occur.

Example 1. Let $\mathrm{G}=\mathrm{GL}(3)$ with simple roots $\alpha$ and $\beta$. Let $\mathrm{H}$ be the subgroup of $\mathrm{G}$ consisting of matrices of the form

$$
\left(\begin{array}{lll}
* & 0 & * \\
0 & * & * \\
0 & 0 & *
\end{array}\right) \quad \text { or } \quad\left(\begin{array}{lll}
0 & * & * \\
* & 0 & * \\
0 & 0 & *
\end{array}\right)
$$


It is easy to see that $\mathrm{G} / \mathrm{H}$ is spherical of rank one and that $\Gamma(\mathrm{G} / \mathrm{H})$ is as follows:

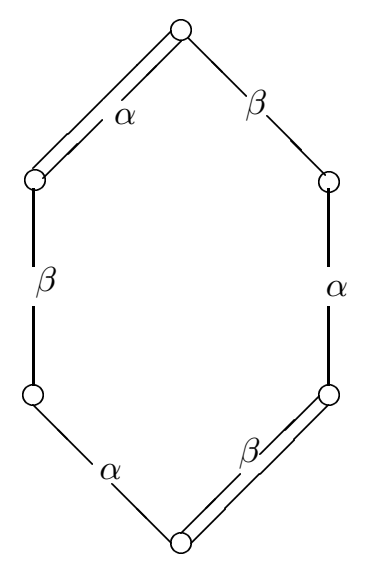

Observe that $\Gamma(\mathrm{G} / \mathrm{H})$ is the same as $\Gamma(\mathrm{G} / \mathrm{B})$, except for double edges. But the geometry of $\mathrm{B}$-orbit closures is very different in both cases: all of them are smooth in $\mathrm{G} / \mathrm{B}$ (the flag variety of $\mathbb{P}^{2}$ ), whereas $\mathrm{G} / \mathrm{H}$ contains a $\mathrm{B}$-stable divisor that is singular in codimension 1. This can be checked directly (see Example 6 for a generalization), or read off the graph of $\mathrm{G} / \mathrm{H}$ as follows.

Let $\mathrm{Z}$ be the closed $\mathrm{B}$-orbit in $\mathrm{G} / \mathrm{H}$. We claim that $\mathrm{Y}=\mathrm{P}_{\beta} \mathrm{P}_{\alpha} \mathrm{Z}$ is singular along $\mathrm{P}_{\alpha} \mathrm{Z}$. Indeed, the morphism $\pi: \mathrm{P}_{\beta} \times{ }^{\mathrm{B}} \mathrm{P}_{\alpha} \mathrm{Z} \rightarrow \mathrm{Y}$ is birational, and $\pi^{-1}\left(\mathrm{P}_{\beta} \mathrm{Z}\right)$ equals $\mathrm{P}_{\beta} \times{ }^{\mathrm{B}} \mathrm{Z}$. But the restriction $\mathrm{P}_{\beta} \times{ }^{\mathrm{B}} \mathrm{Z} \rightarrow \mathrm{P}_{\beta} \mathrm{Z}$ has degree two. Now our claim follows from Zariski's main theorem.

One checks that $r\left(\mathrm{P}_{\beta} \mathrm{Z}\right)=1$, whereas $r(\mathrm{Y})=0$. Thus, the rank function is not compatible with the inclusion order.

Obviously, all closed $\mathrm{B}$-orbits in $\mathrm{G} / \mathrm{H}$ are minimal elements for the weak order. In fact, these closed $\mathrm{B}$-orbits are isomorphic and their codimension is the maximal length of all oriented paths in $\Gamma(\mathrm{G} / \mathrm{H})$, see e.g. [7] 2.2. If moreover $\mathrm{G} / \mathrm{H}$ is symmetric, then all minimal elements of $\Gamma(\mathrm{G} / \mathrm{H})$ are closed orbits, see [20] Theorem 4.6; equivalently, all maximal oriented paths in $\Gamma(\mathrm{G} / \mathrm{H})$ have the same length. But this does not extend to all spherical homogeneous spaces, as shown by

Example 2. Let $\mathrm{G}=\mathrm{GL}(2) \times \mathrm{GL}(2)$ with simple roots $\alpha$ and $\beta$. Let $\mathrm{H}$ be the subgroup of $\mathrm{G}$ consisting of all matrices of the form $(\mathrm{A}, \mathrm{A})$ where $\mathrm{A}$ is upper triangular. One checks easily that $\mathrm{G} / \mathrm{H}$ is spherical with graph 


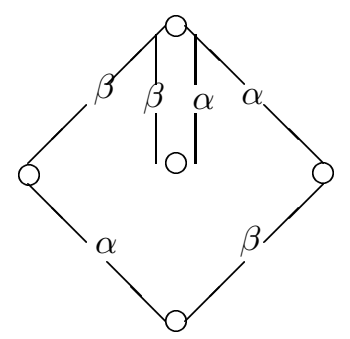

Returning to the general situation, observe that the simple roots of $\mathrm{Y}$ are determined by $\Gamma(\mathrm{G} / \mathrm{H})$ : indeed, $\alpha \in \Delta$ is not in $\Delta(\mathrm{Y})$ if and only if $\alpha$ is the label of an edge with endpoint $\mathrm{Y}$. Similarly, if $\alpha$ raises $\mathrm{Y}$ then its type is determined by $\Gamma(\mathrm{G} / \mathrm{H})$ : it is $\mathrm{U}$ (resp. $\mathrm{N}$ ) if there is a unique edge of label $\alpha$ and target $\mathrm{P}_{\alpha} \mathrm{Y}$ and this edge is simple (resp. double); and it is $\mathrm{T}$ if there are two such edges. It follows that the ranks of $\mathrm{B}$-orbit closures are determined by $\Gamma(\mathrm{G} / \mathrm{H})$ together with $r(\mathrm{G} / \mathrm{H})$.

Example 2 shows that there may be two edges in $\Gamma(\mathrm{G} / \mathrm{H})$ with the same endpoints. In fact, there is no restriction on the number of edges with prescribed endpoints, as shown by the example below suggested by D. Luna. But we shall see that all such edges have the same type.

Example 3. Let $n$ be a positive integer. Let $\mathrm{G}=\mathrm{SL}(2) \times \cdots \times \mathrm{SL}(2) \quad(n$ terms $)$ and let $\mathrm{H}$ be the subgroup of $\mathrm{G}$ consisting of those $n$-tuples $\left(\begin{array}{cc}t & u_{1} \\ 0 & t^{-1}\end{array}\right), \ldots$, $\left(\begin{array}{cc}t & u_{n} \\ 0 & t^{-1}\end{array}\right)$ where $t \in \mathbb{C}^{*}, u_{1}, \ldots, u_{n} \in \mathbb{C}$ and $u_{1}+\cdots+u_{n}=0$. One checks that $\mathrm{G} / \mathrm{H}$ is spherical; the open $\mathrm{H}$-orbit in $\mathrm{G} / \mathrm{B} \cong \mathbb{P}^{1} \times \cdots \times \mathbb{P}^{1}$ ( $n$ terms) consists of those $\left(z_{1}, \ldots, z_{n}\right)$ such that $z_{i} \neq \infty$ for all $i$, and that $z_{1}+\cdots+z_{n} \neq 0$. Let $\mathrm{Y}$ be the $\mathrm{B}$-stable hypersurface in $\mathrm{G} / \mathrm{H}$ corresponding to the $\mathrm{H}$-stable hypersurface $\left(z_{1}+\cdots+z_{n}=0\right)$ in $\mathrm{G} / \mathrm{B}$. One checks that $\mathrm{Y}$ is irreducible and raised to $\mathrm{G} / \mathrm{H}$ by all simple roots of $\mathrm{G}$ (there are $n$ of them). Thus, $\mathrm{Y}$ is joined to $\mathrm{G} / \mathrm{H}$ by $n$ edges of type $\mathrm{T}$.

Proposition 1. Let $\mathrm{Y}, \mathrm{Y}^{\prime} \in \mathcal{B}(\mathrm{G} / \mathrm{H})$ and let $\alpha, \beta$ be distinct simple roots raising $\mathrm{Y}$ to $\mathrm{Y}^{\prime}$. Then either $\alpha, \beta$ are orthogonal and of type $\mathrm{U}$, or they are of type $\mathrm{T}$.

Proof. We begin with two lemmas that reduce the "local" study of $\Gamma(\mathrm{G} / \mathrm{H})$ to simpler situations.

Let $\mathrm{Y} \in \mathcal{B}(\mathrm{G} / \mathrm{H})$ and let $\mathrm{P}=\mathrm{P}_{\mathrm{I}}$ be a standard parabolic subgroup of $\mathrm{G}$, with radical $\mathrm{R}(\mathrm{P})$. Let $\mathcal{B}\left(\mathrm{PY}^{0}\right)$ be the set of all closures in $\mathrm{G} / \mathrm{H}$ of $\mathrm{B}$-orbits in $\mathrm{PY}^{0}$; in other words, $\mathcal{B}\left(\mathrm{PY}^{0}\right)$ is the set of all $\mathrm{Z} \in \mathcal{B}(\mathrm{G} / \mathrm{H})$ such that $\mathrm{PZ}=\mathrm{PY}$. Let $\Gamma\left(\mathrm{PY}^{0}\right)$ be the oriented graph with set of vertices $\mathcal{B}\left(\mathrm{PY}^{0}\right)$, and with edges those edges of $\Gamma(\mathrm{G} / \mathrm{H})$ that have both endpoints in $\mathcal{B}\left(\mathrm{PY}^{0}\right)$ and labels in $\mathrm{I}$. 
Lemma 2. The quotient $\mathrm{PY}^{0} / \mathrm{R}(\mathrm{P})$ exists and is a spherical homogeneous space under $\mathrm{P} / \mathrm{R}(\mathrm{P})$, with graph $\Gamma\left(\mathrm{PY}^{0}\right)$.

Proof. Since $\mathrm{PY}^{0}$ is a unique $\mathrm{P}$-orbit and $\mathrm{R}(\mathrm{P})$ is a normal subgroup of $\mathrm{P}$ contained in $\mathrm{B}$, the quotient $\mathrm{PY}^{0} / \mathrm{R}(\mathrm{P})$ exists and is homogeneous under $\mathrm{P} / \mathrm{R}(\mathrm{P})$; moreover, any $\mathrm{B} / \mathrm{R}(\mathrm{P})$-orbit in $\mathrm{PY}^{0} / \mathrm{R}(\mathrm{P})$ pulls back to a unique $\mathrm{B}$-orbit in $\mathrm{PY}^{0}$. Let $\mathcal{O}$ be a $\mathrm{B}$-orbit in $\mathrm{PY}^{0}$ and let $\alpha \in \mathrm{I}$. Then $\mathrm{R}\left(\mathrm{P}_{\alpha}\right)$ contains $\mathrm{R}(\mathrm{P})$, the square

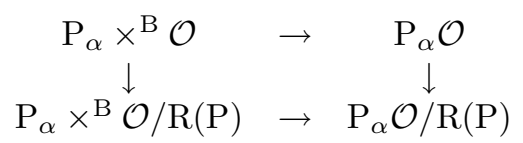

is cartesian, and the map $\mathrm{P}_{\alpha} \times{ }^{\mathrm{B}} \mathcal{O} / \mathrm{R}(\mathrm{P}) \rightarrow \mathrm{P}_{\alpha} / \mathrm{R}(\mathrm{P}) \times{ }^{\mathrm{B} / \mathrm{R}(\mathrm{P})} \mathcal{O} / \mathrm{R}(\mathrm{P})$ is an isomorphism. Thus, the type is preserved under pull back.

Let $\mathrm{H}^{\prime}$ be a closed subgroup of the normalizer $\mathrm{N}_{\mathrm{G}}(\mathrm{H})$ such that $\mathrm{H}^{\prime}$ contains $\mathrm{H}$, and that the quotient $\mathrm{H}^{\prime} / \mathrm{H}$ is connected. Let $\mathrm{Z}(\mathrm{G})$ be the center of $\mathrm{G}$, then $\mathrm{G} / \mathrm{H}^{\prime} \mathrm{Z}(\mathrm{G})$ is a spherical homogeneous space under the adjoint group $\mathrm{G} / \mathrm{Z}(\mathrm{G})$. The natural $\mathrm{G}$-equivariant map $p: \mathrm{G} / \mathrm{H} \rightarrow \mathrm{G} / \mathrm{H}^{\prime} \mathrm{Z}(\mathrm{G})$ is the quotient by the right action of $\mathrm{H}^{\prime} \mathrm{Z}(\mathrm{G})$ on $\mathrm{G} / \mathrm{H}$.

Lemma 3. The pull-back under $p$ of any $\mathrm{B}$-orbit in $\mathrm{G} / \mathrm{H}^{\prime} \mathrm{Z}(\mathrm{G})$ is a unique $\mathrm{B}$-orbit in $\mathrm{G} / \mathrm{H}$. This defines an isomorphism of $\Gamma\left(\mathrm{G} / \mathrm{H}^{\prime} \mathrm{Z}(\mathrm{G})\right.$ ) onto $\Gamma(\mathrm{G} / \mathrm{H})$.

Proof. The first assertion follows from [7] Proposition 2.2 (iii). The second assertion is checked as in the proof of Lemma 2.

Lemma 4. Let $\mathrm{Y} \in \mathcal{B}(\mathrm{G} / \mathrm{H}), \mathrm{Y} \neq \mathrm{G} / \mathrm{H}$, and let $\alpha \in \Delta$. If $\mathrm{P}_{\alpha} \mathrm{Y}^{0}=\mathrm{G} / \mathrm{H}$ then $\alpha$ is orthogonal to $\Delta-\{\alpha\}$, and the derived subgroup of $\mathrm{L}_{\Delta-\{\alpha\}}$ fixes pointwise $\mathrm{G} / \mathrm{H}$.

Proof. We may assume that $\mathrm{G}$ is semi-simple adjoint and acts faithfully on $\mathrm{G} / \mathrm{H}$; then it suffices to check that $G \cong P G L(2)$. Let $H$ be the isotropy group in $G$ of a point of $Y^{0}$. Since $P_{\alpha} Y^{0}=G / H$, we have $P_{\alpha} H=G$. Equivalently, the map $\mathrm{H} / \mathrm{P}_{\alpha} \cap \mathrm{H} \rightarrow \mathrm{G} / \mathrm{P}_{\alpha}$ is an isomorphism. But since $\mathrm{Y} \neq \mathrm{G} / \mathrm{H}$, we have $\mathrm{Y}^{0} \neq \mathrm{P}_{\alpha} \mathrm{Y}^{0}$, so that the image of $\mathrm{P}_{\alpha} \cap \mathrm{H}$ in $\mathrm{P}_{\alpha} / \mathrm{R}\left(\mathrm{P}_{\alpha}\right) \cong \mathrm{PGL}(2)$ is a proper subgroup. It follows that $\left(\mathrm{P}_{\alpha} \cap \mathrm{H}\right)^{0}$ is solvable. Thus, $\mathrm{H} / \mathrm{P}_{\alpha} \cap \mathrm{H}$ is the flag variety of $H^{0}$. Let $\mathrm{S}$ be a maximal semi-simple subgroup of $H^{0}$. Then the rank of $\mathrm{S}$ is the rank of the Picard group of its flag variety $H / \mathrm{P}_{\alpha} \cap \mathrm{H} \cong \mathrm{G} / \mathrm{P}_{\alpha}$, so that $r(\mathrm{~S})=r(\mathrm{G})-1$. Moreover, the connected automorphism group of this flag variety is $\mathrm{S} / \mathrm{Z}(\mathrm{S})$ (see e.g. [9]). Thus, we have a map $\mathrm{G} \rightarrow \mathrm{S} / \mathrm{Z}(\mathrm{S})$ that "splits" the inclusion of $S$ into $G$. It follows that $S$ is a direct factor of $G$, whence $S$ is trivial since $\mathrm{G}$ acts faithfully on $\mathrm{G} / \mathrm{H}$. Thus, $\mathrm{G}=\mathrm{P}_{\alpha} \cong \mathrm{PGL}(2)$. 
We now prove Proposition 1. Applying Lemma 2 to $\mathrm{Y}^{\prime}$ and $\mathrm{P}_{\alpha, \beta}$, we may assume that $\mathrm{Y}^{\prime}=\mathrm{G} / \mathrm{H}$ and that $\Delta=\{\alpha, \beta\}$.

If $\alpha$ has type $\mathrm{U}$, then $r(\mathrm{Y})=r(\mathrm{G} / \mathrm{H})$ whence $\beta$ has type $\mathrm{U}$ as well. We claim that $\mathcal{B}(\mathrm{G} / \mathrm{H})$ consists of $\mathrm{Y}$ and $\mathrm{G} / \mathrm{H}$. Indeed, if $\mathrm{Z} \in \mathcal{B}(\mathrm{G} / \mathrm{H})$ and $\mathrm{Z} \neq \mathrm{G} / \mathrm{H}$, then $\mathrm{Z}$ is connected to $\mathrm{G} / \mathrm{H}$ by an oriented path in $\Gamma(\mathrm{G} / \mathrm{H})$. Consider the top edge of that path; let $\mathrm{Z}^{\prime}$ be its source and (say) $\alpha$ its label. Since $\alpha$ has type $\mathrm{U}$, it follows that $\mathrm{Z}^{\prime}=\mathrm{Y}$. Hence $\mathrm{Z}=\mathrm{Y}$ (for otherwise, $\mathrm{Y}$ would be stable by $\mathrm{P}_{\alpha}$ or $\left.\mathrm{P}_{\beta}\right)$. Thus, $\mathrm{P}_{\alpha} \mathrm{Y}^{0}=\mathrm{G} / \mathrm{H}$; then $\alpha$ and $\beta$ are orthogonal by Lemma 4.

If $\alpha$ has type $\mathrm{N}$, then $r(\mathrm{Y})=r(\mathrm{G} / \mathrm{H})-1$, whence $\beta$ has type $\mathrm{N}$ or $\mathrm{T}$. We consider two subcases.

If $\alpha$ and $\beta$ have type $\mathrm{N}$, we see as above that $\mathrm{G} / \mathrm{H}=\mathrm{P}_{\alpha} \mathrm{Y}^{0}=\mathrm{P}_{\beta} \mathrm{Y}^{0}$. Thus, $\alpha$ and $\beta$ are orthogonal by Lemma 4. Using Lemma 3, we may assume that $\mathrm{G}=\mathrm{PGL}(2) \times \mathrm{PGL}(2)$ and that $\mathrm{H}$ contains a copy of $\mathrm{PGL}(2)$. Then $\mathrm{H}$ is conjugate to PGL(2) embedded diagonally in G. But then both $\alpha$ and $\beta$ have type $\mathrm{T}$, a contradiction.

Finally, if $\alpha$ has type $\mathrm{N}$ and $\beta$ has type $\mathrm{T}$, then there exists $y \in \mathrm{Y}^{0}$ such that $\left(\mathrm{P}_{\beta}\right)_{y}$ is contained in $\mathrm{R}\left(\mathrm{P}_{\beta}\right) \mathrm{T}$. Since the homogeneous spaces $\mathrm{P}_{\beta} / \mathrm{R}\left(\mathrm{P}_{\beta}\right) \mathrm{T}$ and $\mathrm{R}\left(\mathrm{P}_{\beta}\right) \mathrm{T} /\left(\mathrm{P}_{\beta}\right)_{y}$ are affine, the same holds for $\mathrm{P}_{\beta} /\left(\mathrm{P}_{\beta}\right)_{y} \cong \mathrm{P}_{\beta} \mathrm{Y}^{0}$. It follows that $\mathrm{G} / \mathrm{H}-\mathrm{P}_{\beta} \mathrm{Y}^{0}$ is pure of codimension 1 in $\mathrm{G} / \mathrm{H}$. But $\mathrm{P}_{\beta} \mathrm{Y}^{0}$ meets both $\mathrm{B}$-orbits of codimension 1 in $\mathrm{G} / \mathrm{H}$, so that $\mathrm{P}_{\beta} \mathrm{Y}^{0}=\mathrm{G} / \mathrm{H}$. This case is excluded as above. Thus, type $\mathrm{N}$ does not occur.

We next study oriented paths in $\Gamma(\mathrm{G} / \mathrm{H})$. Let $\gamma$ be such a path, with source $\mathrm{Y}$ and target $\mathrm{Y}^{\prime}$. Let $\left(a_{1}, a_{2}, \ldots, a_{\ell}\right)$ be the sequence of labels of edges of $\gamma$, where $\ell=\ell(\gamma)$ is the length of the path. Let $\ell_{\mathrm{U}}(\gamma)\left(\operatorname{resp} . \ell_{\mathrm{T}}(\gamma), \ell_{\mathrm{N}}(\gamma)\right)$ be the number of edges of type $\mathrm{U}$ (resp. T, N) in $\gamma$. Then

$$
\ell_{\mathrm{U}}(\gamma)+\ell_{\mathrm{T}}(\gamma)+\ell_{\mathrm{N}}(\gamma)=\ell(\gamma)=\operatorname{dim}\left(\mathrm{Y}^{\prime}\right)-\operatorname{dim}(\mathrm{Y})
$$

Define an element $w(\gamma)$ of $\mathrm{W}$ by $w(\gamma)=s_{\alpha_{\ell}} \cdots s_{\alpha_{2}} s_{\alpha_{1}}$.

Lemma 5. (i) $s_{\alpha_{\ell}} \cdots s_{\alpha_{2}} s_{\alpha_{1}}$ is a reduced decomposition of $w(\gamma)$; equivalently, $\ell(w(\gamma))=\ell$.

(ii) $\ell_{\mathrm{T}}(\gamma)+\ell_{\mathrm{N}}(\gamma)=r\left(\mathrm{Y}^{\prime}\right)-r(\mathrm{Y})$. In particular, $\ell_{\mathrm{T}}(\gamma)+\ell_{\mathrm{N}}(\gamma)$ and $\ell_{\mathrm{U}}(\gamma)$ depend only on the endpoints of $\gamma$.

(iii) The morphism $\mathrm{G} \times{ }^{\mathrm{B}} \mathrm{Y} \rightarrow \mathrm{G} / \mathrm{H}:(g, y) \mathrm{B} \rightarrow$ gy restricts to a morphism $\overline{\mathrm{B} w(\gamma) \mathrm{B}} \times{ }^{\mathrm{B}} \mathrm{Y} \rightarrow \mathrm{Y}^{\prime}$ that is surjective and generically finite of degree $2^{\ell_{\mathrm{N}}(\gamma)}$. In particular, $\ell_{\mathrm{T}}(\gamma)$ and $\ell_{\mathrm{N}}(\gamma)$ depend only on the endpoints of $\gamma$ and on $w(\gamma)$. Moreover, $w(\gamma)$ is in $\mathrm{W}^{\Delta(\mathrm{Y})}$, and $w(\gamma)^{-1}$ is in $\mathrm{W}^{\Delta\left(\mathrm{Y}^{\prime}\right)}$.

(iv) If $\mathrm{H}$ is contained in a Borel subgroup of $\mathrm{G}$, then $\ell_{\mathrm{N}}(\gamma)=0$ so that $\ell_{\mathrm{T}}(\gamma)$ depends only on the endpoints of $\gamma$.

Proof. (i) Observe that $\mathrm{B} s_{\alpha_{1}} \mathrm{Y}$ is dense in $\mathrm{P}_{\alpha_{1}} \mathrm{Y}$, as $\alpha_{1}$ raises $\mathrm{Y}$. By induction, it follows that $\mathrm{B} s_{\alpha_{\ell}} \mathrm{B} \cdots s_{\alpha_{2}} \mathrm{~B} s_{\alpha_{1}} \mathrm{Y}$ is dense in $\mathrm{Y}^{\prime}$. Because $\operatorname{dim}\left(\mathrm{Y}^{\prime}\right)=\operatorname{dim}(\mathrm{Y})+\ell$, 
we must have $\operatorname{dim}\left(\overline{\mathrm{B} s_{\alpha_{\ell}} \mathrm{B} \cdots s_{\alpha_{2}} \mathrm{~B} s_{\alpha_{1}} \mathrm{~B}} / \mathrm{B}\right)=\ell$, whence $\ell\left(s_{\alpha_{\ell}} \cdots s_{\alpha_{2}} s_{\alpha_{1}}\right)=\ell$.

(ii) follows from the fact that $r\left(\mathrm{Y}^{\prime}\right)=r(\mathrm{Y})($ resp. $r(\mathrm{Y})+1)$ if $\mathrm{Y}$ is the source of an edge with target $\mathrm{Y}^{\prime}$ and type $\mathrm{U}$ (resp. T, N).

(iii) By (i), the product maps

$$
\mathrm{P}_{\alpha_{i}} \times{ }^{\mathrm{B}} \cdots \times{ }^{\mathrm{B}} \mathrm{P}_{\alpha_{2}} \times{ }^{\mathrm{B}} \mathrm{P}_{\alpha_{1}} \rightarrow \overline{\mathrm{B} s_{\alpha_{i}} \cdots s_{\alpha_{2}} s_{\alpha_{1}} \mathrm{~B}}
$$

are birational for $1 \leq i \leq \ell$. It follows that the morphism $\overline{\mathrm{B} w(\gamma) \mathrm{B}} \times{ }^{\mathrm{B}} \mathrm{Y} \rightarrow \mathrm{G} / \mathrm{H}$ has image $\mathrm{Y}^{\prime}$; moreover, its degree is the product of the degrees of the

$$
\pi_{i}: \mathrm{P}_{\alpha_{i}} \times{ }^{\mathrm{B}}\left(\mathrm{P}_{\alpha_{i-1}} \cdots \mathrm{P}_{\alpha_{1}} \mathrm{Y}\right) \rightarrow \mathrm{P}_{\alpha_{i}} \mathrm{P}_{\alpha_{i-1}} \cdots \mathrm{P}_{\alpha_{1}} \mathrm{Y}
$$

that is, $2^{\ell_{\mathrm{N}}(\gamma)}$.

Let $w=w(\gamma)$. We show that $w^{-1} \in \mathrm{W}^{\Delta\left(\mathrm{Y}^{\prime}\right)}$. Otherwise, there exists $\alpha \in \Delta\left(\mathrm{Y}^{\prime}\right)$ such that $\ell\left(s_{\alpha} w\right)=\ell(w)-1$. Thus, $\mathrm{B} w \mathrm{~B}=\mathrm{B} s_{\alpha} \mathrm{B} s_{\alpha} w \mathrm{~B}$, and $\mathrm{Y}^{\prime}=\overline{\mathrm{B} w \mathrm{Y}}=\overline{\mathrm{B} s_{\alpha} \mathrm{B} s_{\alpha} w \mathrm{Y}}$. Let $\mathrm{Y}^{\prime \prime}=\overline{\mathrm{B} s_{\alpha} w \mathrm{Y}}$, then $\alpha$ raises $\mathrm{Y}^{\prime \prime}$ to $\mathrm{Y}^{\prime}$. This contradicts the assumption that $\alpha \in \Delta\left(\mathrm{Y}^{\prime}\right)$. A similar argument shows that $w \in \mathrm{W}^{\Delta(\mathrm{Y})}$.

(iv) If $\ell_{\mathrm{N}}(\gamma)>0$, then there exists a point $x \in \mathrm{G} / \mathrm{H}$, a simple root $\alpha$ and a surjective group homomorphism $\left(\mathrm{P}_{\alpha}\right)_{x} \rightarrow \mathrm{N}$ where $\mathrm{N}$ is the normalizer of a torus in $\mathrm{PGL}(2)$. Since $\mathrm{N}$ consists of semi-simple elements, it is a quotient of $\left(\mathrm{P}_{\alpha}\right)_{x} / \mathrm{R}_{u}\left(\mathrm{P}_{\alpha}\right)_{x}$. By assumption, the latter is isomorphic to a subgroup of $\mathrm{B} / \mathrm{U}=\mathrm{T}$. Thus, $\mathrm{N}$ is abelian, a contradiction.

Corollary 1. Let $\mathrm{Y}, \mathrm{Y}^{\prime} \in \mathcal{B}(\mathrm{G} / \mathrm{H})$, then $\mathrm{Y} \preceq \mathrm{Y}^{\prime}$ if and only if there exists $w \in \mathrm{W}$ such that $\mathrm{Y}^{\prime}=\overline{\mathrm{B} w \mathrm{Y}}$.

Proof. Recall that $\overline{\mathrm{B} w \mathrm{~B}}$ (closure in $\mathrm{G}$ ) is a product of minimal parabolic subgroups. Thus, $\mathrm{Y} \preceq \overline{\mathrm{B} w \mathrm{~B} Y}=\overline{\mathrm{B} w \mathrm{Y}}$. The converse has just been proved.

For later use, we study the behavior of $\Gamma(\mathrm{G} / \mathrm{H})$ under parabolic induction in the following sense (see [6] 1.2.) Let $\mathrm{P}=\mathrm{P}_{\mathrm{I}}$ be a standard parabolic subgroup with Levi subgroup $\mathrm{L}=\mathrm{L}_{\mathrm{I}}$ and let $\mathrm{H}^{\prime}$ be a spherical subgroup of $\mathrm{L}$, then the induced homogeneous space is $\mathrm{G} / \mathrm{R}_{u}(\mathrm{P}) \mathrm{H}^{\prime}$. In other words, $\mathrm{G} / \mathrm{H}$ is the total space of the homogeneous bundle over $\mathrm{G} / \mathrm{P}$ with fiber $\mathrm{L} / \mathrm{H}^{\prime}$. By [loc. cit.], each $\mathrm{Y} \in \mathcal{B}(\mathrm{G} / \mathrm{H})$ can be written uniquely as $\overline{\mathrm{B} w \mathrm{Y}^{\prime}}$ for $w \in \mathrm{W}^{\mathrm{I}}$ and $\mathrm{Y}^{\prime} \in \mathcal{B}\left(\mathrm{L} / \mathrm{H}^{\prime}\right)$; then $r(\mathrm{Y})=r\left(\mathrm{Y}^{\prime}\right)$. We thus identify $\mathcal{B}(\mathrm{G} / \mathrm{H})$ to $\mathrm{W}^{\mathrm{I}} \times \mathcal{B}\left(\mathrm{L} / \mathrm{H}^{\prime}\right)$. The next result describes the edges of $\Gamma(\mathrm{G} / \mathrm{H})$ in terms of those of $\Gamma\left(\mathrm{L} / \mathrm{H}^{\prime}\right)$.

Lemma 6. Let $\alpha \in \Delta, w \in \mathrm{W}^{\mathrm{I}}$ and $\mathrm{Y}^{\prime} \in \mathcal{B}\left(\mathrm{L} / \mathrm{H}^{\prime}\right)$; let $\beta=w^{-1}(\alpha)$. Then the edges of $\Gamma(\mathrm{G} / \mathrm{H})$ with source $\left(w, \mathrm{Y}^{\prime}\right)$ and label $\alpha$ are as follows:

(i) If $\beta \in \Phi^{+}-\mathrm{I}$, join $\left(w, \mathrm{Y}^{\prime}\right)$ to $\left(s_{\alpha} w, \mathrm{Y}^{\prime}\right)$ by an edge of type $\mathrm{U}$.

(ii) If $\beta \in \mathrm{I}$ and $\beta$ raises $\mathrm{Y}^{\prime}$, join $\left(w, \mathrm{Y}^{\prime}\right)$ to $\left(w,\left(\mathrm{P}_{\beta} \cap \mathrm{L}\right) \mathrm{Y}^{\prime}\right)$ by an edge of the same type as the edge from $\mathrm{Y}^{\prime}$ to $\left(\mathrm{P}_{\beta} \cap \mathrm{L}\right) \mathrm{Y}^{\prime}$.

Proof. Since $w \in \mathrm{W}^{\mathrm{I}}$, we have $s_{\alpha} w \in \mathrm{W}^{\mathrm{I}}$ if and only if $\beta \notin \mathrm{I}$. In that case, $\alpha$ 
raises $\mathrm{Y}$ if and only if $\ell\left(s_{\alpha} w\right)=\ell(w)+1$, that is, $\beta \in \Phi^{+}$. Then $\mathrm{P}_{\alpha} \mathrm{Y}=\overline{\mathrm{B} s_{\alpha} w \mathrm{Y}^{\prime}}$ and the map $\pi_{\mathrm{Y}, \alpha}$ is the pull-back of $\pi_{\overline{\mathrm{B} w \mathrm{P}} / \mathrm{P}, \alpha}$ under the map $\overline{\mathrm{B} w \mathrm{Y}^{\prime}} \rightarrow \overline{\mathrm{B} w \mathrm{P}} / \mathrm{P}$. This yields case (i).

But if $\beta \in \mathrm{I}$, then $s_{\alpha} w=w s_{\beta}$ has length $\ell(w)+1$, so that

$$
\mathrm{P}_{\alpha} \mathrm{Y}=\overline{\mathrm{B} s_{\alpha} \mathrm{B} w \mathrm{Y}^{\prime}}=\overline{\mathrm{B} s_{\alpha} w \mathrm{Y}^{\prime}}=\overline{\mathrm{B} w s_{\beta} \mathrm{Y}^{\prime}}=\overline{\mathrm{B} w \mathrm{~B} s_{\beta} \mathrm{Y}^{\prime}}=\overline{\mathrm{B} w\left(\mathrm{P}_{\beta} \cap \mathrm{L}\right) \mathrm{Y}^{\prime}} .
$$

Thus, $\alpha$ raises $\mathrm{Y}$ if and only if $\beta$ raises $\mathrm{Y}^{\prime}$. Then, as $s_{\alpha} w=w s_{\beta}$, we can join $\mathrm{Y}^{\prime}$ to $\mathrm{P}_{\alpha} \mathrm{Y}$ by two paths: one beginning with $\ell(w)$ edges of type $\mathrm{U}$ followed by an edge from $\mathrm{Y}$ to $\mathrm{P}_{\alpha} \mathrm{Y}$, and another one beginning with an edge from $\mathrm{Y}^{\prime}$ to $\left(\mathrm{P}_{\beta} \cap \mathrm{L}\right) \mathrm{Y}^{\prime}$ followed by $\ell(w)$ edges of type $\mathrm{U}$. Using Lemma 5 , this yields case (ii).

For instance, Example 1 is obtained from $\mathrm{SL}(2) / \mathrm{N}$ by parabolic induction.

Returning to the general case, we shall see in Proposition 5 that the numbers $\ell_{\mathrm{T}}(\gamma)$ and $\ell_{\mathrm{N}}(\gamma)$ depend only on the endpoints of the oriented path $\gamma$, if $\mathrm{G}$ is simply-laced (that is, if all roots have the same length for an appropriate choice of the $\mathrm{W}$-invariant scalar product on $X$; equivalently, $\Phi$ is a product of irreducible root systems of type A, D or E.) This assumption cannot be omitted, as shown by

Example 4. Let $\mathrm{G}=\mathrm{SP}(4)$ be the subgroup of GL(4) preserving a nondegenerate symplectic form, and let $\mathrm{H}=\mathrm{GL}(2)$ be the subgroup of $\mathrm{G}$ preserving two complementary lagrangian planes. The normalizer $\mathrm{N}_{\mathrm{G}}(\mathrm{H})$ contains $\mathrm{H}$ as a subgroup of index 2 . The graph $\Gamma(\mathrm{G} / \mathrm{H})$ is as follows:

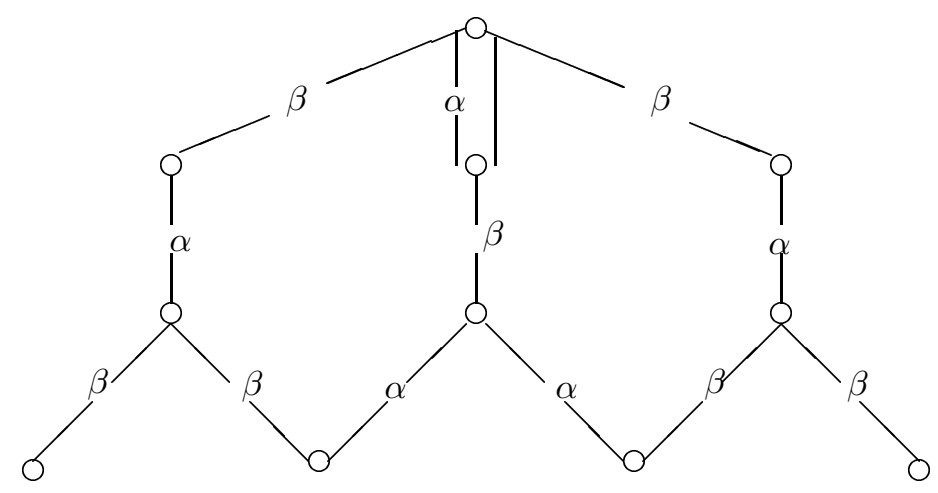


And here is $\Gamma\left(\mathrm{G} / \mathrm{N}_{\mathrm{G}}(\mathrm{H})\right)$ :

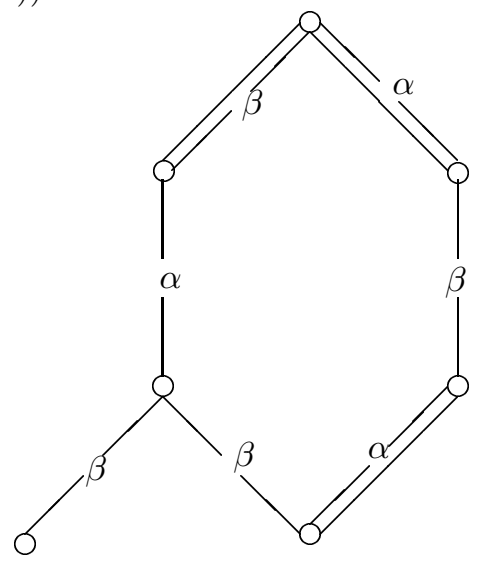

Using parabolic induction, one constructs similar examples for $\Phi$ of type B, C or $\mathrm{F}$.

To proceed, we need the following definition taken from [6]:

Definition. For $\mathrm{Y} \in \mathcal{B}(\mathrm{G} / \mathrm{H})$, let $\mathrm{W}(\mathrm{Y})$ be the set of all $w \in \mathrm{W}$ such that the morphism $\pi_{\mathrm{Y}, w}: \overline{\mathrm{B} w \mathrm{~B}} \times{ }^{\mathrm{B}} \mathrm{Y} \rightarrow \mathrm{GY}$ is surjective and generically finite. For $w \in \mathrm{W}(\mathrm{Y})$, let $d(\mathrm{Y}, w)$ be the degree of $\pi_{\mathrm{Y}, w}$.

By Lemma 5, W(Y) consists of all $w(\gamma)$ where $\gamma$ is an oriented path from $\mathrm{Y}$ to $\mathrm{GY}$; moreover, $d(\mathrm{Y}, w(\gamma))=2^{\ell_{\mathrm{N}}(\gamma)}$, and $w^{-1} \in \mathrm{W}^{\Delta(\mathrm{G} / \mathrm{H})}$ for all $w \in \mathrm{W}(\mathrm{Y})$.

We now introduce a notion of neighbors in $\mathrm{W}(\mathrm{Y})$, and we show that any two elements of that set are connected by a chain of neighbors. Let $\alpha, \beta$ be distinct simple roots and let $m$ be a positive integer. Let

$$
\left(s_{\alpha} s_{\beta}\right)^{(m)}=\cdots s_{\beta} s_{\alpha} s_{\beta} s_{\alpha} \quad(m \text { terms. })
$$

Then we have the braid relation $\left(s_{\alpha} s_{\beta}\right)^{(m(\alpha, \beta))}=\left(s_{\beta} s_{\alpha}\right)^{(m(\alpha, \beta))}$, where $m(\alpha, \beta)$ denotes the order of $s_{\alpha} s_{\beta}$ in W.

Definition. Two elements $u$ and $v$ of $\mathrm{W}$ are neighbors if there exist $x, y$ in $\mathrm{W}$ together with distinct $\alpha, \beta$ in $\Delta$ and a positive integer $m<m(\alpha, \beta)$ such that

$$
u=x\left(s_{\alpha} s_{\beta}\right)^{(m)} y, v=x\left(s_{\beta} s_{\alpha}\right)^{(m)} y, \quad \text { and } \quad \ell(u)=\ell(x)+m+\ell(y)=\ell(v) .
$$

For example, any two simple reflections are neighbors, since $s_{\alpha}=\left(s_{\alpha} s_{\beta}\right)^{(1)}$.

Proposition 2. Let $\mathrm{Y} \in \mathcal{B}(\mathrm{G} / \mathrm{H})$ and let $u, v$ be distinct elements of $\mathrm{W}(\mathrm{Y})$. Then there exists a sequence $\left(u=u_{0}, u_{1}, \ldots, u_{n}=v\right)$ in $\mathrm{W}(\mathrm{Y})$ such that each $u_{i+1}$ is a neighbor of $u_{i}$. 
Proof. By induction on $\ell(u)=\ell(v)=\ell$, the case where $\ell=1$ being evident.

If there exists $\alpha \in \Delta$ such that $\ell\left(u s_{\alpha}\right)=\ell\left(v s_{\alpha}\right)=\ell-1$, then $\alpha$ raises $\mathrm{Y}$, and $u s_{\alpha}, v s_{\alpha}$ are in $\mathrm{W}\left(\mathrm{P}_{\alpha} \mathrm{Y}\right)$. Now the induction assumption for $\mathrm{P}_{\alpha} \mathrm{Y}$ concludes the proof in this case.

Otherwise, we can find distinct $\alpha, \beta \in \Delta$ such that $\ell\left(u s_{\alpha}\right)=\ell\left(v s_{\beta}\right)=\ell-1$. Then $\mathrm{P}_{\alpha}$ and $\mathrm{P}_{\beta}$ raise $\mathrm{Y}$ to subvarieties of $\mathrm{P}_{\alpha, \beta} \mathrm{Y}$. Let $m$ be the common codimension of $\mathrm{P}_{\alpha} \mathrm{Y}$ and $\mathrm{P}_{\beta} \mathrm{Y}$ in $\mathrm{P}_{\alpha, \beta} \mathrm{Y}$, then we have

$$
\mathrm{P}_{\alpha, \beta} \mathrm{Y}=\cdots \mathrm{P}_{\alpha} \mathrm{P}_{\beta} \mathrm{P}_{\alpha} \mathrm{Y}=\overline{\mathrm{B} \cdots s_{\alpha} s_{\beta} s_{\alpha} \mathrm{Y}} \quad(m \text { terms })
$$

Choose $x \in \mathrm{W}\left(\mathrm{P}_{\alpha, \beta} \mathrm{Y}\right)$, then $\mathrm{W}(\mathrm{Y})$ contains $x\left(s_{\alpha} s_{\beta}\right)^{(m)}$ and, similarly, $x\left(s_{\beta} s_{\alpha}\right)^{(m)}$, as neighbors. Moreover, $\mathrm{W}\left(\mathrm{P}_{\alpha} \mathrm{Y}\right)$ contains $u s_{\alpha}$ and $x\left(s_{\beta} s_{\alpha}\right)^{(m-1)}$, whereas $\mathrm{W}\left(\mathrm{P}_{\beta} \mathrm{Y}\right)$ contains $x\left(s_{\beta} s_{\alpha}\right)^{(m-1)}$ and $v s_{\beta}$. Now we conclude by the induction assumption for $\mathrm{P}_{\alpha} \mathrm{Y}$ and $\mathrm{P}_{\beta} \mathrm{Y}$.

Neighbors in $\mathrm{W}$ are also close to each other for the Bruhat-Chevalley order $\leq$ :

Proposition 3. For any neighbors $u, v \in \mathrm{W}$ such that $u^{-1}, v^{-1} \in \mathrm{W}^{\Delta(\mathrm{G} / \mathrm{H})}$, there exists $w \in \mathrm{W}$ such that $u \leq w, v \leq w, w^{-1} \in \mathrm{W}^{\Delta(\mathrm{G} / \mathrm{H})}$ and $\ell(w)=$ $\ell(u)+1=\ell(v)+1$.

Proof. Write $u=x\left(s_{\alpha} s_{\beta}\right)^{(m)} y$ and $v=x\left(s_{\beta} s_{\alpha}\right)^{(m)} y$. Let

$$
w=x\left(s_{\alpha} s_{\beta}\right)^{(m)} s_{\beta} y .
$$

We claim that $\ell(w)$ equals $\ell(x)+m+1+\ell(y)=\ell(u)+1=\ell(v)+1$. Otherwise, $\ell(w) \leq \ell(x)+\ell(y)+m-1<l(u)$ and $w=u y^{-1} s_{\beta} y=u s_{y^{-1}(\beta)}$. By the strong exchange condition ([13] Theorem 5.8 applied to $u$ ), one of the following cases occurs:

(i) $w=x^{\prime}\left(s_{\alpha} s_{\beta}\right)^{(m)} y$ where $\ell\left(x^{\prime}\right)=\ell(x)-1$. Comparing both expressions for $w$, we obtain $x^{\prime}\left(s_{\alpha} s_{\beta}\right)^{(m)}=x\left(s_{\alpha} s_{\beta}\right)^{(m)} s_{\beta}$. Thus, there exists $\gamma \in \Phi_{\alpha, \beta}^{+}$ such that $x^{\prime}=x s_{\gamma}$. But $\ell\left(x s_{\alpha}\right)=\ell\left(x s_{\beta}\right)=\ell(x)+1$, for $\ell\left(x\left(s_{\alpha} s_{\beta}\right)^{(m)} y\right)=$ $\ell\left(x\left(s_{\beta} s_{\alpha}\right)^{(m)} y\right)=\ell(x)+m+\ell(y)$. It follows that $x(\alpha)$ and $x(\beta)$ are in $\Phi^{+}$. Thus, $x \in \mathrm{W}^{\alpha, \beta}$. Since $s_{\gamma} \in \mathrm{W}_{\alpha, \beta}$, we have $\ell\left(x^{\prime}\right)=\ell(x)+\ell\left(s_{\gamma}\right) \geq \ell(x)$, a contradiction.

(ii) $w=x z y$ where $z$ is obtained from $\left(s_{\alpha} s_{\beta}\right)^{(m)}$ by deleting a simple reflection. Then the equality $z=\left(s_{\alpha} s_{\beta}\right)^{(m)} s_{\beta}$ leads to a braid relation of length at most $m<m(\alpha, \beta)$, a contradiction.

(iii) $w=x\left(s_{\alpha} s_{\beta}\right)^{(m)} y^{\prime}$ where $\ell\left(y^{\prime}\right)=\ell(y)-1$. Then $y^{\prime}=s_{\beta} y$. But $\ell\left(s_{\beta} y\right)=$ $\ell(y)+1$, for $\ell(v)=\ell(x)+m+\ell(y)$; a contradiction.

By the claim and [13] Theorem 5.10, we have $u \leq w$ and $v \leq w$. Write $w=$ $w^{\prime \prime} w^{\prime}$ where $w^{\prime \prime} \in \mathrm{W}_{\Delta(\mathrm{G} / \mathrm{H})}$ and $\left(w^{\prime}\right)^{-1} \in \mathrm{W}^{\Delta(\mathrm{G} / \mathrm{H})}$; then $\ell(w)=\ell\left(w^{\prime}\right)+\ell\left(w^{\prime \prime}\right)$. Since $u^{-1} \leq w^{-1}$ and $u^{-1} \in \mathrm{W}^{\Delta(\mathrm{G} / \mathrm{H})}$, it follows that $u^{-1} \leq\left(w^{\prime}\right)^{-1}$ by [10] Lemma 3.5. Thus, $u \leq w^{\prime}$ and $v \leq w^{\prime}$. Since $u \neq v$ and $\ell(u)=\ell(v)=$ $\ell(w)-1 \geq \ell\left(w^{\prime}\right)-1$, we must have $w=w^{\prime}$, so that $w^{-1} \in \mathrm{W}^{\Delta(\mathrm{G} / \mathrm{H})}$. 
Recall that $r(\mathrm{Y}) \leq r(\mathrm{G} / \mathrm{H})$ for any $\mathrm{Y} \in \mathcal{B}(\mathrm{G} / \mathrm{H})$. If equality holds, then neighbors in $\mathrm{W}(\mathrm{Y})$ have a very simple form:

Proposition 4. Let $\mathrm{Y} \in \mathcal{B}(\mathrm{G} / \mathrm{H})$ such that $r(\mathrm{Y})=r(\mathrm{G} / \mathrm{H})$; let $u, v \in \mathrm{W}(\mathrm{Y})$ be neighbors. Then $u=x s_{\alpha} y$ and $v=x s_{\beta} y$ where $x, y \in \mathrm{W}$ and $\alpha, \beta$ are orthogonal simple roots such that $\ell(u)=\ell(v)=\ell(x)+\ell(y)+1$. Moreover, $X(\mathrm{G} / \mathrm{H})$ contains $x(\alpha+\beta)$.

Proof. Write $u=x\left(s_{\alpha} s_{\beta}\right)^{(m)} y$ and $v=x\left(s_{\beta} s_{\alpha}\right)^{(m)} y$ as in the definition of neighbors. Then $x\left(s_{\alpha} s_{\beta}\right)^{(m)}$ and $x\left(s_{\beta} s_{\alpha}\right)^{(m)}$ are neighbors in $\mathrm{W}(\overline{\mathrm{B} y \mathrm{Y}})$. Moreover, $r(\overline{\mathrm{B} y \mathrm{Y}}) \geq r(\mathrm{Y})$, whence $r(\overline{\mathrm{B} y \mathrm{Y}})=r(\mathrm{G} / \mathrm{H})$. Thus, we may assume that $y=1$.

Let $\mathrm{Y}^{\prime}=\overline{\mathrm{B}\left(s_{\alpha} s_{\beta}\right)^{(m)} \mathrm{Y}}$ and $\mathrm{Y}^{\prime \prime}=\overline{\mathrm{B}\left(s_{\beta} s_{\alpha}\right)^{(m)} \mathrm{Y}}$, then we obtain similarly: $r\left(\mathrm{Y}^{\prime}\right)=r\left(\mathrm{Y}^{\prime \prime}\right)=r(\mathrm{G} / \mathrm{H})$ and $x \in \mathrm{W}\left(\mathrm{Y}^{\prime}\right) \cap \mathrm{W}\left(\mathrm{Y}^{\prime \prime}\right)$. If $x \neq 1$, write $x=s_{\gamma} x^{\prime}$ where $\gamma \in \Delta$ and $\ell(x)=\ell\left(x^{\prime}\right)+1$. Then $\overline{\mathrm{B} x^{\prime} \mathrm{Y}^{\prime}}$ and $\overline{\mathrm{B} x^{\prime} \mathrm{Y}^{\prime \prime}}$ have rank $r(\mathrm{G} / \mathrm{H})$ and are raised to $\mathrm{G} / \mathrm{H}$ by $\gamma$. Thus, $\gamma$ has type $\mathrm{U}$, so that $\overline{\mathrm{B} x^{\prime} \mathrm{Y}^{\prime}}=\overline{\mathrm{B} x^{\prime} \mathrm{Y}^{\prime \prime}}$. By induction on $\ell(x)$, we obtain $\mathrm{Y}^{\prime}=\mathrm{Y}^{\prime \prime}$. This subvariety is stable under $\mathrm{P}_{\alpha, \beta}$. Applying Lemmas 2 and 3, we may assume that $\mathrm{Y}^{\prime}=\mathrm{G} / \mathrm{H}$ (i.e., $x=1$ ), $\Delta=\{\alpha, \beta\}$, the center of $\mathrm{G}$ is trivial, and $\mathrm{H}$ has finite index in its normalizer. Moreover, we have $\mathrm{P}(\mathrm{G} / \mathrm{H})=\mathrm{B}$, for $\mathrm{P}_{\alpha}$ and $\mathrm{P}_{\beta}$ do not stabilize $(\mathrm{G} / \mathrm{H})^{0}$.

We claim that any $\mathrm{Z} \in \mathcal{B}(\mathrm{G} / \mathrm{H})$ can be written as

$$
\overline{\mathrm{B}\left(s_{\alpha} s_{\beta}\right)^{(n)} \mathrm{Y}}=\cdots \mathrm{P}_{\beta} \mathrm{P}_{\alpha} \mathrm{Y} \quad \text { or } \quad \overline{\mathrm{B}\left(s_{\beta} s_{\alpha}\right)^{(n)} \mathrm{Y}}=\cdots \mathrm{P}_{\alpha} \mathrm{P}_{\beta} \mathrm{Y} \quad(n \text { terms }),
$$

where $n=\operatorname{dim}(\mathrm{Z})-\operatorname{dim}(\mathrm{Y})$ satisfies $0 \leq n \leq m$. For this, we argue by induction on the codimension of $\mathrm{Z}$ in $\mathrm{G} / \mathrm{H}$. We may assume that $\alpha$ raises $\mathrm{Z}$. By the induction assumption, we have

$$
\mathrm{P}_{\alpha} \mathrm{Z}=\mathrm{P}_{\beta} \mathrm{P}_{\alpha} \cdots \mathrm{Y} \quad \text { or } \quad \mathrm{P}_{\alpha} \mathrm{Z}=\mathrm{P}_{\alpha} \mathrm{P}_{\beta} \cdots \mathrm{Y} \quad(n+1 \text { terms }) .
$$

In the latter case, let $\mathrm{Z}^{\prime}=\mathrm{P}_{\beta} \cdots \mathrm{Y}\left(n\right.$ terms). Since $\mathrm{P}_{\alpha} \mathrm{Z}=\mathrm{P}_{\alpha} \mathrm{Z}^{\prime}$ and $r(\mathrm{Z})=$ $r\left(\mathrm{Z}^{\prime}\right)=r\left(\mathrm{P}_{\alpha} \mathrm{Z}\right)=r(\mathrm{Y})$, it follows that $\mathrm{Z}=\mathrm{Z}^{\prime}$. In the former case, $\mathrm{P}_{\alpha} \mathrm{Z}$ is stable under $\mathrm{G}$ and hence equal to $\mathrm{G} / \mathrm{H}$; in particular, $\mathrm{Z}$ has codimension $1 \mathrm{in} \mathrm{G} / \mathrm{H}$. Now $\mathrm{G} / \mathrm{H}=\mathrm{P}_{\alpha} \mathrm{P}_{\beta} \cdots \mathrm{Y}$ ( $m$ terms), so that we are in the previous case.

By the claim, all $\mathrm{B}$-orbit closures in $\mathrm{G} / \mathrm{H}$ have the same rank, and $\mathrm{Y}^{0}$ is the unique closed B-orbit. Let $y \in \mathrm{Y}^{0}$; we may assume that $\mathrm{H}=\mathrm{G}_{y}$. Since the $\mathrm{H}$-orbit in $\mathrm{G} / \mathrm{B}$ corresponding to the $\mathrm{B}$-orbit $\mathrm{Y}^{0}$ in $\mathrm{G} / \mathrm{H}$ is closed, the connected isotropy group $\mathrm{B}_{y}^{0}$ is a Borel subgroup of $H^{0}$. It follows that $r(\mathrm{Y})=$ $r(\mathrm{~B})-r\left(\mathrm{~B}_{y}\right)=2-r(\mathrm{H})$. On the other hand, $r(\mathrm{Y})=r(\mathrm{G} / \mathrm{H})$ by assumption. Thus, $r(\mathrm{G} / \mathrm{H})=2-r(\mathrm{H})$.

If $r(\mathrm{G} / \mathrm{H})=0$ then $\mathrm{H}$ is a parabolic subgroup of $\mathrm{G}$ (in fact, a Borel subgroup as $\mathrm{P}(\mathrm{G} / \mathrm{H})=\mathrm{B}$.) Moreover, $\mathrm{Y}$ is the $\mathrm{B}$-fixed point in $\mathrm{G} / \mathrm{H}$. But then $\mathrm{W}(\mathrm{Y})$ consists of a unique element (of maximal length in $\mathrm{W}$ ), a contradiction.

If $r(\mathrm{G} / \mathrm{H})=1$ then $r(\mathrm{H})=1$ as well. Using the classification of homogeneous spaces of rank 1 under semi-simple groups of rank 2 (see e.g. Table 1 of [26]), this forces $\mathrm{G}=\mathrm{PGL}(2) \times \mathrm{PGL}(2)$ and $\mathrm{H}=\mathrm{PGL}(2)$ embedded diagonally in $\mathrm{G}$. 
As a consequence, the simple roots $\alpha$ and $\beta$ are orthogonal, and $X(\mathrm{G} / \mathrm{H})$ is generated by $\alpha+\beta$.

If $r(\mathrm{G} / \mathrm{H})=2$ then $r(\mathrm{H})=0$, that is, $H^{0}$ is unipotent. Since $\mathrm{G} / \mathrm{H}$ is spherical, $H^{0}$ is a maximal unipotent subgroup of $\mathrm{G}$. This contradicts the assumption that $\mathrm{H}$ has finite index in its normalizer.

Proposition 5. If $\mathrm{G}$ is simply-laced, then

(i) for any oriented path $\gamma$ in $\Gamma(\mathrm{G} / \mathrm{H})$, both $\ell_{\mathrm{T}}(\gamma)$ and $\ell_{\mathrm{N}}(\gamma)$ depend only on the endpoints of $\gamma$.

(ii) for any $\mathrm{Y} \in \mathcal{B}(\mathrm{G} / \mathrm{H})$, there exists an oriented path $\gamma$ joining $\mathrm{Y}$ to $\mathrm{G} / \mathrm{H}$ through a sequence of simple edges followed by a sequence of double edges.

Proof. (i) Let Y (resp. $\mathrm{Y}^{\prime}$ ) be the source (resp. target) of $\gamma$, and let $\delta$ be another oriented path from $\mathrm{Y}$ to $\mathrm{Y}^{\prime}$. By Lemma 5 , it suffices to show that $\ell_{\mathrm{N}}(\gamma)=\ell_{\mathrm{N}}(\delta)$. Joining $\mathrm{Y}^{\prime}$ to $\mathrm{G} / \mathrm{H}$ by an oriented path, we reduce to the case where $\mathrm{Y}^{\prime}=\mathrm{G} / \mathrm{H}$; then $w(\gamma)$ and $w(\delta)$ are in $\mathrm{W}(\mathrm{Y})$. By Proposition 2, we may assume moreover that $w(\gamma)$ and $w(\delta)$ are neighbors. Using Lemmas 2 and 3, we reduce to the case where the center of $\mathrm{G}$ is trivial, $\Delta=\{\alpha, \beta\}, \mathrm{H}$ has finite index in its normalizer, $w(\gamma)=\left(s_{\alpha} s_{\beta}\right)^{(m)}$ and $w(\delta)=\left(s_{\beta} s_{\alpha}\right)^{(m)}$ for some $m<m(\alpha, \beta)$.

Since $\mathrm{G}$ is simply-laced, we have either $\mathrm{G}=\mathrm{PGL}(2) \times \mathrm{PGL}(2)$ and $m(\alpha, \beta)=$ 2 , or $\mathrm{G}=\operatorname{PGL}(3)$ and $m(\alpha, \beta)=3$. In particular, $m \leq 2$. If $m=1$ then $\ell_{\mathrm{N}}(\gamma)=\ell_{\mathrm{N}}(\delta)=0$ by Proposition 1 . If $m=2$ then $\mathrm{G}=\mathrm{PGL}(3)$. Using Lemma 5 (iv), we may assume moreover that $\mathrm{H}$ is not contained in any Borel subgroup. Then we see by inspection that $\mathrm{H}$ is conjugate to $\mathrm{PO}(3)$ or to $\mathrm{GL}(2)$.

In the latter case, here is $\Gamma(\mathrm{G} / \mathrm{H})$ :

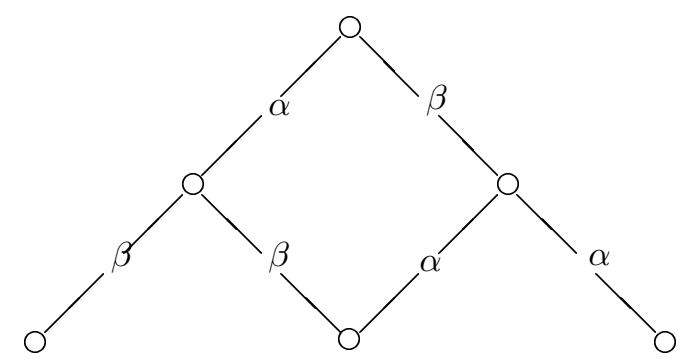

Thus, $\ell_{\mathrm{N}}(\gamma)=\ell_{\mathrm{N}}(\delta)=0$. 
In the former case, we have $\ell_{\mathrm{N}}(\gamma)=\ell_{\mathrm{N}}(\delta)=1$, since $\Gamma(\mathrm{G} / \mathrm{H})$ is as follows:

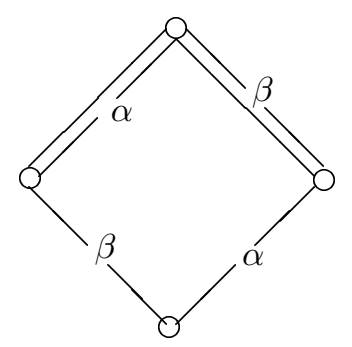

(ii) Let $\gamma$ be an oriented path joining $\mathrm{Y}$ to $\mathrm{G} / \mathrm{H}$. We may assume that $\gamma$ contains double edges. Consider the lowest maximal subpath $\delta$ of $\gamma$ that consists only of double edges; we may assume that the endpoint of $\delta$ is not $\mathrm{G} / \mathrm{H}$. Let $\mathrm{Y}^{\prime}$ be the source of the top edge of $\delta$, and let $\alpha$ (resp. $\beta$ ) be the label of that edge (resp. of the next edge of $\gamma$, a simple edge by assumption.) We claim that there exists an oriented path $\gamma^{\prime}$ joining $\mathrm{Y}^{\prime}$ to $\mathrm{G} / \mathrm{H}$ and beginning with a simple edge; then assertion (ii) will follow by induction on $\ell(\delta)+\operatorname{codim}_{\mathrm{G} / \mathrm{H}}\left(\mathrm{Y}^{\prime}\right)$.

To check the claim, it suffices to join $\mathrm{Y}^{\prime}$ to $\mathrm{P}_{\alpha \beta} \mathrm{Y}^{\prime}$ by an oriented path $\gamma^{\prime}$ beginning with a simple edge. As above, we reduce to the case where $\mathrm{G}$ equals $\mathrm{PGL}(2) \times \mathrm{PGL}(2)$ or $\mathrm{PGL}(3)$, and $\mathrm{H}$ is not contained in a Borel subgroup of $\mathrm{G}$; moreover, $\mathrm{H}$ has finite index in its normalizer. Using the fact that $\Gamma(\mathrm{G} / \mathrm{H})$ contains a double edge followed by a simple edge, one checks that $\mathrm{H}$ is a product of subgroups of $\mathrm{PGL}(2)$ if $\mathrm{G}=\operatorname{PGL}(2) \times \operatorname{PGL}(2)$; and if $\mathrm{G}=\operatorname{PGL}(3)$, then $\mathrm{H}$ is conjugate to the subgroup of Example 1, or to its transpose. The path $\gamma^{\prime}$ exists in all of these cases.

From Proposition 5, we will deduce a criterion for the graph of $\mathrm{G} / \mathrm{H}$ to contain only simple edges. To formulate it, we need more notation and a preliminary result.

Let $\mathcal{D}(\mathrm{G} / \mathrm{H})$ be the subset of $\mathcal{B}(\mathrm{G} / \mathrm{H})$ consisting of all irreducible $\mathrm{B}$-stable divisors. The elements of $\mathcal{D}(\mathrm{G} / \mathrm{H})$ are called colors; they play an important role in the classification of embeddings of $\mathrm{G} / \mathrm{H}$, see [15]. Let $\mathrm{D} \in \mathcal{D}(\mathrm{G} / \mathrm{H})$, with preimage $\tilde{D}$ in $G$. Replacing $G$ by a finite cover, we may assume that $\tilde{D}$ is the divisor of a regular function $f_{\mathrm{D}}$ on $\mathrm{G}$. Then $f_{\mathrm{D}}$ is an eigenvector of $\mathrm{B}$ acting by left multiplication; let $\omega_{\mathrm{D}}$ be its weight. Since $f_{\mathrm{D}}$ is uniquely defined up to multiplication by a regular invertible function on $G$, then $\omega_{D}$ is unique up to addition of a character of $\mathrm{G}$. In particular, for any $\alpha \in \Delta$, the number $\left\langle\omega_{\mathrm{D}}, \check{\alpha}\right\rangle$ is a non-negative integer depending only on $\mathrm{D}$ and $\alpha$.

Lemma 7. The degree $d(\mathrm{D}, \alpha)$ of the morphism $\pi_{\mathrm{D}, \alpha}: \mathrm{P}_{\alpha} \times{ }^{\mathrm{B}} \mathrm{D} \rightarrow \mathrm{G} / \mathrm{H}$ equals $\left\langle\omega_{\mathrm{D}}, \check{\alpha}\right\rangle$ if $\pi_{\mathrm{D}, \alpha}$ is generically finite; otherwise, $\left\langle\omega_{\mathrm{D}}, \check{\alpha}\right\rangle=0$.

Proof. Note that $\mathrm{D}$ is $\mathrm{P}_{\alpha}$-stable if and only if $f_{\mathrm{D}}$ is an eigenvector of $\mathrm{P}_{\alpha}$, that 
is, $\omega_{\mathrm{D}}$ extends to a character of that group. This amounts to: $\left\langle\omega_{\mathrm{D}}, \check{\alpha}\right\rangle=0$.

Let $\mathrm{V}$ be the $\mathrm{H}$-stable divisor in $\mathrm{G} / \mathrm{B}$ corresponding to the $\mathrm{B}$-stable divisor $\mathrm{D}$ in $\mathrm{G} / \mathrm{H}$. Then $\mathrm{V}$ is the zero scheme of a section of the homogeneous line bundle on $\mathrm{G} / \mathrm{B}$ associated with the character $\omega_{\mathrm{D}}$ of $\mathrm{B}$. Let $p: \mathrm{G} / \mathrm{B} \rightarrow \mathrm{G} / \mathrm{P}_{\alpha}$ be the natural map, then $d(\mathrm{D}, \alpha)$ equals the degree of the restriction $p_{\mathrm{V}}: \mathrm{V} \rightarrow \mathrm{G} / \mathrm{P}_{\alpha}$. The latter degree is the intersection number of $\mathrm{V}$ with a fiber of $p$, that is, $\left\langle\omega_{\mathrm{D}}, \check{\alpha}\right\rangle$.

A direct consequence of Lemma 7 and Proposition 5 is

Corollary 2. For simply-laced $\mathrm{G}$, the following conditions are equivalent:

(i) Each edge of $\Gamma(\mathrm{G} / \mathrm{H})$ is simple.

(ii) For any $\mathrm{D} \in \mathcal{D}(\mathrm{G} / \mathrm{H})$ and $\alpha \in \Delta$, we have $\left\langle\omega_{\mathrm{D}}, \check{\alpha}\right\rangle \leq 1$.

It follows e.g. that the graphs of the symmetric spaces $\operatorname{GL}(p+q) / \mathrm{GL}(p) \times$ $\mathrm{GL}(q), \mathrm{SL}(2 n) / \mathrm{SP}(2 n), \mathrm{SO}(2 n) / \mathrm{GL}(n)$ and $\mathrm{E}_{6} / \mathrm{F}_{4}$ contain only simple edges. For this, one uses the explicit description of colors of symmetric spaces given in [25].

Note that Corollary 2 does not extend to multiply-laced groups G. Consider, for example, $\mathrm{G}=\mathrm{SO}(2 n+1)$ and its subgroup $\mathrm{H}=\mathrm{O}(2 n)$, the stabilizer of a non-degenerate line in $\mathbb{C}^{2 n+1}$. Then $\mathrm{G} / \mathrm{H}$ is symmetric of rank 1 and its graph consists of a unique oriented path: a double edge followed by $n-1$ simple edges.

\section{Orbit closures in regular completions}

Recall from [2] that a variety $\mathrm{X}$ with an action of $\mathrm{G}$ is called regular if it satisfies the following three conditions:

(i) $\mathrm{X}$ is smooth and contains a dense $\mathrm{G}$-orbit whose complement is a union of irreducible smooth divisors (the boundary divisors) with normal crossings.

(ii) Any $\mathrm{G}$-orbit closure in $\mathrm{X}$ is the transversal intersection of those boundary divisors that contain it.

(iii) For any $x \in \mathrm{X}$, the normal space to the orbit $\mathrm{G} x$ contains a dense orbit of the isotropy group of $x$.

If moreover $\mathrm{X}$ is complete and its dense $\mathrm{G}$-orbit is isomorphic to $\mathrm{G} / \mathrm{H}$, we call $\mathrm{X}$ a regular completion of that homogeneous space. Recall from [3] that $\mathrm{G} / \mathrm{H}$ admits a regular completion if and only if it is spherical; then any equivariant completion of $\mathrm{G} / \mathrm{H}$ is the image of a regular one by an equivariant morphism.

We fix a regular completion $\mathrm{X}$ and we denote by $\mathcal{B}(\mathrm{X})$ (resp. $\mathcal{B}(\mathrm{G} / \mathrm{H}, \mathrm{X}))$ the set of all $\mathrm{B}$-orbit closures in $\mathrm{X}$ (resp. of those that meet G/H.) By [6] 1.4, any $\mathrm{Y} \in \mathcal{B}(\mathrm{G} / \mathrm{H}, \mathrm{X})$ meets all $\mathrm{G}$-orbit closures properly. Moreover, for any closed $\mathrm{G}$ orbit $\mathrm{Z}$, the irreducible components of $\mathrm{Y} \cap \mathrm{Z}$ are the Schubert varieties $\overline{\mathrm{B} w^{-1} z}$ where $w \in \mathrm{W}(\mathrm{Y})$ and $z$ is the unique $\mathrm{T}$-fixed point of $\mathrm{Z}$ such that $\overline{\mathrm{B} z}=\mathrm{Z}$. 
This point $z$ will be called the base point of $\mathrm{Z}$; its isotropy group is opposed to $\mathrm{P}(\mathrm{G} / \mathrm{H})$, see e.g. [3] 2.2. The intersection multiplicity of $\mathrm{Y}$ and $\mathrm{Z}$ along $\overline{\mathrm{B} w^{-1} z}$ equals $d(\mathrm{Y}, w)$.

We shall generalize this to intersections of $\mathrm{Y}$ with arbitrary $\mathrm{G}$-orbit closures. For this, we recall the local structure of $X$ (see e.g. [3] 2.3). Set $P=P(G / H)$ and $\mathrm{L}=\mathrm{L}(\mathrm{G} / \mathrm{H})$. Let $\mathrm{X}_{0}$ be the set of all $x \in \mathrm{X}$ such that $\mathrm{B} x$ is open in $\mathrm{G} x$. Then $\mathrm{X}_{0}$ is an open $\mathrm{P}$-stable subset of $\mathrm{X}$. Moreover, there exists an L-stable subvariety $\mathrm{S}$ of $\mathrm{X}_{0}$, fixed pointwise by $[\mathrm{L}, \mathrm{L}]$, such that the map

$$
\begin{aligned}
\mathrm{R}_{u}(\mathrm{P}) \times \mathrm{S} & \rightarrow \mathrm{X}_{0} \\
(g, x) & \mapsto g x
\end{aligned}
$$

is an isomorphism. As a consequence, $\mathrm{S}$ is a smooth toric variety (for a quotient of $\mathrm{T}$ ) of dimension $r(\mathrm{G} / \mathrm{H})$, the rank of $\mathrm{G} / \mathrm{H}$; moreover, $\mathrm{S}$ meets each $\mathrm{G}$-orbit along a unique $\mathrm{T}$-orbit. Let $\varphi: \mathrm{X}_{0} \cong \mathrm{R}_{u}(\mathrm{P}) \times \mathrm{S} \rightarrow \mathrm{S}$ be the second projection, then $\varphi$ is L-equivariant; it can be seen as the quotient map by the action of $\mathrm{R}_{u}(\mathrm{P})$.

Choose $w \in \mathrm{W}(\mathrm{Y})$, then $w \mathrm{Y}$ meets $\mathrm{X}_{0}$ (because $\overline{\mathrm{B} w \mathrm{Y}}=\mathrm{X}$ ), and the intersection $\mathrm{X}_{0} \cap w \mathrm{Y}$ is stable by the group $\mathrm{P} \cap w \mathrm{~B} w^{-1}$. The latter contains $\mathrm{R}_{u}(\mathrm{P}) \cap w \mathrm{U}^{-1}$ as a normal subgroup. We shall see that $\mathrm{R}_{u}(\mathrm{P}) \cap w \mathrm{U}^{-1}$ acts freely on $w \mathrm{Y} \cap \mathrm{X}_{0}$, with section

$$
\mathrm{S}_{\mathrm{Y}, w}=w \mathrm{Y} \cap\left(\mathrm{U} \cap w \mathrm{U}^{-} w^{-1}\right) \mathrm{S} .
$$

Note that $\mathrm{U} \cap w \mathrm{U}^{-} w^{-1}$ is contained in $\mathrm{R}_{u}(\mathrm{P})$, because $w^{-1} \in \mathrm{W}^{\mathrm{P}}$. Thus, $\mathrm{S}_{\mathrm{Y}, w}$ is a closed $\mathrm{T}$-stable subvariety of $w \mathrm{Y} \cap \mathrm{X}_{0}$. Let

$$
\varphi_{\mathrm{Y}, w}: \mathrm{S}_{\mathrm{Y}, w} \rightarrow \mathrm{S}
$$

be the restriction of $\varphi: \mathrm{X}_{0} \rightarrow \mathrm{S}$, then $\varphi_{\mathrm{Y}, w}$ is $\mathrm{T}$-equivariant.

Proposition 6. Keep notation as above.

(i) The map

$$
\begin{aligned}
\left(\mathrm{R}_{u}(\mathrm{P}) \cap w \mathrm{U}^{-1}\right) \times \mathrm{S}_{\mathrm{Y}, w} & \rightarrow w \mathrm{Y} \cap \mathrm{X}_{0} \\
(g, x) & \mapsto g x
\end{aligned}
$$

is an isomorphism.

(ii) The variety $\mathrm{S}_{\mathrm{Y}, w}$ is irreducible and meets each $\mathrm{G}$-orbit along a unique $\mathrm{T}$ orbit. In particular, $\mathrm{S}_{\mathrm{Y}, w} \cap \mathrm{G} / \mathrm{H}$ is a unique $\mathrm{T}$-orbit, dense in $\mathrm{S}_{\mathrm{Y}, w}$ and contained in $w \mathrm{Y}^{0}$; and $\mathrm{S}_{\mathrm{Y}, w} \cap \mathrm{Z}=\{z\}$ for any closed $\mathrm{G}$-orbit $\mathrm{Z}$ with base point $z$.

(iii) The morphism $\varphi_{\mathrm{Y}, w}$ is finite surjective of degree $d(\mathrm{Y}, w)$. As a consequence, the dimension of $\mathrm{S}_{\mathrm{Y}, w}$ is the rank of $\mathrm{X}$.

Proof. (i) The product map $\left(\mathrm{R}_{u}(\mathrm{P}) \cap w \mathrm{U}^{-1}\right) \times\left(\mathrm{R}_{u}(\mathrm{P}) \cap w \mathrm{U}^{-} w^{-1}\right) \rightarrow \mathrm{R}_{u}(\mathrm{P})$ is an isomorphism; moreover, $\mathrm{R}_{u}(\mathrm{P}) \cap w \mathrm{U}^{-} w^{-1}=\mathrm{U} \cap w \mathrm{U}^{-} w^{-1}$. Therefore, the 
product map

$$
\left(\mathrm{R}_{u}(\mathrm{P}) \cap w \mathrm{U}^{-1}\right) \times\left(\mathrm{U} \cap w \mathrm{U}^{-} w^{-1}\right) \mathrm{S} \rightarrow \mathrm{X}_{0}
$$

is an isomorphism. The assertion follows by intersecting with $w \mathrm{Y}$.

(ii) and (iii) The union of all $\mathrm{G}$-orbits in $\mathrm{X}$ that contain $\mathrm{Z}$ in their closure is a $\mathrm{G}$-stable open subset of $\mathrm{X}$. Thus, we may assume that $\mathrm{Z}$ is the unique closed $\mathrm{G}$ orbit in $\mathrm{X}$. Let $\mathrm{D}_{1}, \ldots, \mathrm{D}_{r}$ be the boundary divisors, then $r=r(\mathrm{X})$. Moreover, $\mathrm{S}$ is isomorphic to affine space $\mathbb{A}^{r}$ with coordinate functions $x_{1}, \ldots, x_{r}$, equations of $\mathrm{D}_{1} \cap \mathrm{S}, \ldots, \mathrm{D}_{r} \cap \mathrm{S}$. The compositions $f_{1}=x_{1} \circ \varphi, \ldots, f_{r}=x_{r} \circ \varphi$ are equations of $\mathrm{D}_{1} \cap \mathrm{X}_{0}, \ldots, \mathrm{D}_{r} \cap \mathrm{X}_{0}$; they generate the ideal of $\mathrm{Z} \cap \mathrm{X}_{0}=\mathrm{B} z$ in $\mathrm{X}_{0}$. The map $\varphi: \mathrm{X}_{0} \rightarrow \mathrm{S}$ identifies to $\left(f_{1}, \ldots, f_{r}\right): \mathrm{X}_{0} \rightarrow \mathbb{A}^{r}$. The intersections of $\mathrm{G}$-orbit closures with $\mathrm{X}_{0}$ are the pull-backs of coordinate subspaces of $\mathbb{A}^{r}$.

By (i), $\mathrm{S}_{\mathrm{Y}, w}$, is irreducible. We check that $\mathrm{S}_{\mathrm{Y}, w} \cap \mathrm{Z}=\{z\}$. For this, note that the product map

$$
\left(\mathrm{R}_{u}(\mathrm{P}) \cap w \mathrm{U}^{-1}\right) \times\left(\mathrm{S}_{\mathrm{Y}, w} \cap \mathrm{Z}\right) \rightarrow w \mathrm{Y} \cap \mathrm{X}_{0} \cap \mathrm{Z}=w \mathrm{Y} \cap \mathrm{B} z
$$

is an isomorphism. Moreover, since $\mathrm{Y}$ meets $\mathrm{Z}$ properly, with $\overline{\mathrm{B} w^{-1} z}$ as an irreducible component, it follows that $w \mathrm{Y} \cap \mathrm{B} z$ is equidimensional, with

$$
\overline{w \mathrm{~B} w^{-1} z} \cap \mathrm{B} z=\left(\mathrm{B} \cap w \mathrm{~B} w^{-1}\right) z
$$

as an irreducible component. The latter is isomorphic to $\mathrm{R}_{u}(\mathrm{P}) \cap w \mathrm{U} w^{-1}$. Thus, the $\mathrm{T}$-stable set $\mathrm{S}_{Y, w} \cap \mathrm{Z}$ is finite, so that it consists of $\mathrm{T}$-fixed points. Since $z$ is the unique $\mathrm{T}$-fixed point in $\mathrm{B} z$, our assertion follows.

The map $\varphi_{\mathrm{Y}, w}: \mathrm{S}_{\mathrm{Y}, w} \rightarrow \mathrm{S}$ identifies with $\left(f_{1}, \ldots, f_{r}\right): \mathrm{S}_{\mathrm{Y}, w} \rightarrow \mathbb{A}^{r}$. We just saw that the set-theoretical fiber of 0 is $\{z\}$. Since 0 is the unique closed $\mathrm{T}$-orbit in $\mathbb{A}^{r}$, all fibers of $\varphi_{\mathrm{Y}, w}$ are finite. Thus, $\mathrm{S}_{\mathrm{Y}, w}$ contains a dense $\mathrm{T}$-orbit. Since $\mathrm{S}_{\mathrm{Y}, w}$ is affine and contains a $\mathrm{T}$-fixed point $z$, it follows that $\varphi_{\mathrm{Y}, w}$ is finite and that the pull-back of any $\mathrm{T}$-orbit in $\mathrm{S}$ is a unique $\mathrm{T}$-orbit. This implies (ii).

Finally, we check that the degree of $\varphi_{\mathrm{Y}, w}$ equals $d(\mathrm{Y}, w)$, that is, the degree of the natural map $\overline{\mathrm{B} w \mathrm{~B}} \times{ }^{\mathrm{B}} \mathrm{Y} \rightarrow \mathrm{X}$. For this, note that the map

$$
\mathrm{U} \cap w \mathrm{U}^{-} w^{-1} \rightarrow \overline{\mathrm{B} w \mathrm{~B}} / \mathrm{B}, \quad g \rightarrow g w \mathrm{~B} / \mathrm{B}
$$

is an open immersion. Thus, $d(\mathrm{Y}, w)$ is the degree of the product map ( $\mathrm{U} \cap$ $\left.w \mathrm{U}^{-} w^{-1}\right) \times w \mathrm{Y} \rightarrow \mathrm{X}$, or, equivalently, of its restriction

$$
p:\left(\mathrm{U} \cap w \mathrm{U}^{-} w^{-1}\right) \times\left(w \mathrm{Y} \cap \mathrm{X}_{0}\right) \rightarrow \mathrm{X}_{0} .
$$

The latter map fits into a commutative diagram

$$
\begin{array}{ccc}
\left(\mathrm{U} \cap w \mathrm{U}^{-} w^{-1}\right) \times\left(w \mathrm{Y} \cap \mathrm{X}_{0}\right) & \rightarrow \mathrm{X}_{0} \\
\downarrow & & \downarrow \\
\mathrm{S}_{\mathrm{Y}, w} & \rightarrow \mathrm{S}
\end{array}
$$

where the bottom horizontal map is $\varphi_{\mathrm{Y}, w}$; indeed,

$$
\left(\mathrm{U} \cap w \mathrm{U}^{-} w^{-1}\right) \times\left(w \mathrm{Y} \cap \mathrm{X}_{0}\right) \cong\left(\mathrm{R}_{u}(\mathrm{P}) \cap w \mathrm{U}^{-} w^{-1}\right) \times\left(\mathrm{R}_{u}(\mathrm{P}) \cap w \mathrm{U}^{-1}\right) \times \mathrm{S}_{\mathrm{Y}, w}
$$


by (i). Moreover, the fibers of the right (resp. left) vertical map are isomorphic to $\mathrm{R}_{u}(\mathrm{P})$ (resp. to $\left(\mathrm{R}_{u}(\mathrm{P}) \cap w \mathrm{U}^{-} w^{-1}\right) \times\left(\mathrm{R}_{u}(\mathrm{P}) \cap w \mathrm{U}^{-1}\right) \cong \mathrm{R}_{u}(\mathrm{P})$.) Thus, the diagram is cartesian, and the degree of $p$ equals the degree of $\varphi_{\mathrm{Y}, w}$.

Thus, we can view $\mathrm{S}_{\mathrm{Y}, w}$ as a "slice" in $w \mathrm{Y}$ to $w \mathrm{~B} w^{-1} z=\left(\mathrm{R}_{u}(\mathrm{P}) \cap w \mathrm{U} w^{-1}\right) z$ at $z$. But $\mathrm{S}_{Y, w}$ may be non transversal to $\mathrm{Z}$ at $z$ : indeed, the intersection multiplicity of $\mathrm{S}_{\mathrm{Y}, w}$ and $\mathrm{Z}$ at $z$ equals the intersection multiplicity of $\mathrm{Y}$ and $\mathrm{Z}$ along $\overline{\mathrm{B} w^{-1} z}$, and the latter equals $d(\mathrm{Y}, w)$ by [6] 1.4 (alternatively, this can be deduced from Proposition 6 (iii).) On the other hand, it is not clear whether $\mathrm{S}_{\mathrm{Y}, w}$ is smooth, that is, $\mathrm{Y} \cap w^{-1} \mathrm{X}_{0}$ consists of smooth points of $\mathrm{Y}$; see Corollary 3 below for a partial answer to this question.

We now relate the "slices" associated with both endpoints of an edge in $\Gamma(\mathrm{G} / \mathrm{H})$. Let $\mathrm{Y} \in \mathcal{B}(\mathrm{G} / \mathrm{H}, \mathrm{X})$ and let $\alpha \in \Delta$ raising $\mathrm{Y}$. Choose $v \in \mathrm{W}\left(\mathrm{P}_{\alpha} \mathrm{Y}\right)$, then $w=v s_{\alpha}$ is in $\mathrm{W}(\mathrm{Y})$, and $\ell(w)=\ell(v)+1$. Thus, $v(\alpha) \in \Phi^{+} \cap w\left(\Phi^{-}\right)$. Let $\mathrm{U}_{v(\alpha)}$ be the corresponding unipotent subgroup of dimension 1 , then $\mathrm{U}_{v(\alpha)}$ is contained in $\mathrm{R}_{u}(\mathrm{P}) \cap v \mathrm{U} v^{-1}$.

Proposition 7. With notation as above, $\mathrm{S}_{\mathrm{Y}, w}$ is contained in $\mathrm{U}_{v(\alpha)} \mathrm{S}_{\mathrm{P}_{\alpha} \mathrm{Y}, v}$ and the latter is isomorphic to $\mathrm{U}_{v(\alpha)} \times \mathrm{S}_{\mathrm{P}_{\alpha} \mathrm{Y}, \tau}$. Denoting by

$$
\varphi_{\mathrm{Y}, \alpha}: \mathrm{S}_{\mathrm{Y}, w} \rightarrow \mathrm{S}_{\mathrm{P}_{\alpha} \mathrm{Y}, v}
$$

the corresponding projection, then $\varphi_{\mathrm{Y}, w}=\varphi_{\mathrm{P}_{\alpha} \mathrm{Y}, v} \circ \varphi_{\mathrm{Y}, \alpha}$. Moreover, $\varphi_{\mathrm{Y}, \alpha}$ is finite surjective of degree $d(\mathrm{Y}, \alpha)$.

Proof. We have

$$
\begin{aligned}
\mathrm{S}_{\mathrm{Y}, w} & =w \mathrm{Y} \cap\left(\mathrm{U} \cap w \mathrm{U}^{-} w^{-1}\right) \mathrm{S}=w \mathrm{Y} \cap \mathrm{U}_{v(\alpha)}\left(\mathrm{U} \cap v \mathrm{U}^{-} v^{-1}\right) \mathrm{S} \\
& \subseteq v \mathrm{P}_{\alpha} \mathrm{Y} \cap \mathrm{U}_{v(\alpha)}\left(\mathrm{U} \cap v \mathrm{U}^{-} v^{-1}\right) \mathrm{S}=\mathrm{U}_{v(\alpha)}\left(v \mathrm{P}_{\alpha} \mathrm{Y} \cap\left(\mathrm{U} \cap v \mathrm{U}^{-} v^{-1}\right) \mathrm{S}\right)= \\
& =\mathrm{U}_{v(\alpha)} \mathrm{S}_{\mathrm{P}_{\alpha} \mathrm{Y}, v} .
\end{aligned}
$$

Moreover, since $\mathrm{U}_{v(\alpha)} \subseteq \mathrm{R}_{u}(\mathrm{P}) \cap v \mathrm{U} v^{-1}$, the product map $\mathrm{U}_{v(\alpha)} \times \mathrm{S}_{\mathrm{P}_{\alpha} \mathrm{Y}, v} \rightarrow$ $\mathrm{U}_{v(\alpha)} \mathrm{S}_{\mathrm{P}_{\alpha} \mathrm{Y}, v}$ is an isomorphism. Now the equality $\varphi_{\mathrm{Y}, w}=\varphi_{\mathrm{P}_{\alpha} \mathrm{Y}, v} \circ \varphi_{\mathrm{Y}, \alpha}$ follows from the definitions. Together with Proposition 6 (iii), it implies that $\varphi_{\mathrm{Y}, \alpha}$ is finite surjective of degree $d(\mathrm{Y}, w) d\left(\mathrm{P}_{\alpha} \mathrm{Y}, v\right)^{-1}=d(\mathrm{Y}, \alpha)$.

Next we describe the intersection of a B-orbit closure with an arbitrary Gorbit closure, generalizing [6] Theorem 1.4.

Theorem 1. Let $\mathrm{X}$ be a regular completion of $\mathrm{G} / \mathrm{H}$, let $\mathrm{Y} \in \mathcal{B}(\mathrm{G} / \mathrm{H}, \mathrm{X})$ and let $\mathrm{X}^{\prime}$ be a $\mathrm{G}$-orbit closure in $\mathrm{X}$. Then $\mathrm{W}(\mathrm{Y})$ is the disjoint union of the $\mathrm{W}\left(\mathrm{Y}^{\prime}\right)$ where $\mathrm{Y}^{\prime}$ runs over all irreducible components of $\mathrm{Y} \cap \mathrm{X}^{\prime}$. Moreover, for any such $\mathrm{Y}^{\prime}$ and $w \in \mathrm{W}\left(\mathrm{Y}^{\prime}\right)$, we have

$$
d(\mathrm{Y}, w)=d\left(\mathrm{Y}^{\prime}, w\right) i\left(\mathrm{Y}^{\prime}, \mathrm{Y} \cdot \mathrm{X}^{\prime} ; \mathrm{X}\right)
$$


where $i\left(\mathrm{Y}^{\prime}, \mathrm{Y} \cdot \mathrm{X}^{\prime} ; \mathrm{X}\right)$ denotes the intersection multiplicity of $\mathrm{Y}$ and $\mathrm{X}^{\prime}$ along $\mathrm{Y}^{\prime}$ in $\mathrm{X}$. As a consequence, this multiplicity is a power of 2.

Proof. By [6] Lemma 1.3, $\mathrm{W}(\mathrm{Y})$ is the union of the $\mathrm{W}\left(\mathrm{Y}^{\prime}\right)$. Choose $\mathrm{Y}^{\prime}$ and $w \in \mathrm{W}\left(\mathrm{Y}^{\prime}\right)$, then $\mathrm{Y}^{\prime} \cap w^{-1} \mathrm{X}_{0}$ is an irreducible component of $\mathrm{Y} \cap w^{-1} \mathrm{X}_{0} \cap \mathrm{X}^{\prime}$. The latter is isomorphic to $\left(\mathrm{U} \cap w^{-1} \mathrm{R}_{u}(\mathrm{P})\right) \times w^{-1}\left(\mathrm{~S}_{\mathrm{Y}, w} \cap \mathrm{X}^{\prime}\right)$, and $\mathrm{S}_{\mathrm{Y}, w} \cap \mathrm{X}^{\prime}$ is a unique $\mathrm{T}$-orbit, by Proposition 6. It follows that $\mathrm{Y} \cap w^{-1} \mathrm{X}_{0} \cap \mathrm{X}^{\prime}=\mathrm{Y}^{\prime} \cap w^{-1} \mathrm{X}_{0}$ is irreducible, so that $\mathrm{Y}^{\prime}$ is uniquely determined by $w$. Equivalently, the $\mathrm{W}\left(\mathrm{Y}^{\prime}\right)$ are pairwise disjoint.

Let $\mathrm{Z}$ be a closed $\mathrm{G}$-orbit in $\mathrm{X}^{\prime}$, then

$d(\mathrm{Y}, w)=i\left(\overline{\mathrm{B} w^{-1} z}, \mathrm{Y} \cdot \mathrm{Z} ; \mathrm{X}\right)=i\left(\overline{\mathrm{B} w^{-1} z} \cap w^{-1} \mathrm{X}_{0},\left(\mathrm{Y} \cap w^{-1} \mathrm{X}_{0}\right) \cdot\left(\mathrm{Z} \cap w^{-1} \mathrm{X}_{0}\right) ; w^{-1} \mathrm{X}_{0}\right)$,

where the former equality follows from [6] 1.4, and the latter from [12] 8.2. Moreover, we have by Proposition 6: $\overline{\mathrm{B} w^{-1} z} \cap w^{-1} \mathrm{X}_{0}=\mathrm{B} w^{-1} z$ and $\mathrm{Z} \cap w^{-1} \mathrm{X}_{0}=$ $w^{-1} \mathrm{~B} z$. Thus,

$$
d(\mathrm{Y}, w)=i\left(\mathrm{~B} w^{-1} z,\left(\mathrm{Y} \cap w^{-1} \mathrm{X}_{0}\right) \cdot w^{-1} \mathrm{~B} z, w^{-1} \mathrm{X}_{0}\right) .
$$

Using the fact that $\mathrm{Y} \cap w^{-1} \mathrm{X}_{0} \cap \mathrm{X}^{\prime}=\mathrm{Y}^{\prime} \cap w^{-1} \mathrm{X}_{0}$ is irreducible, together with associativity of intersection multiplicities (see [12] 7.1.8), we obtain

$$
\begin{aligned}
d(\mathrm{Y}, w) & =i\left(\mathrm{~B} w^{-1} z,\left(\mathrm{Y}^{\prime} \cap w^{-1} \mathrm{X}_{0}\right) \cdot w^{-1} \mathrm{~B} z ; w^{-1} \mathrm{X}_{0} \cap \mathrm{X}^{\prime}\right) i\left(\mathrm{Y}^{\prime}, \mathrm{Y} \cdot \mathrm{X}^{\prime} ; \mathrm{X}\right) \\
& =i\left(\overline{\mathrm{B} w^{-1} z}, \mathrm{Y}^{\prime} \cdot \mathrm{Z} ; \mathrm{X}^{\prime}\right) i\left(\mathrm{Y}^{\prime}, \mathrm{Y} \cdot \mathrm{X}^{\prime} ; \mathrm{X}\right)=d\left(\mathrm{Y}^{\prime}, w\right) i\left(\mathrm{Y}^{\prime}, \mathrm{Y} \cdot \mathrm{X}^{\prime} ; \mathrm{X}\right) .
\end{aligned}
$$

These results motivate the following

Definition. A B-orbit closure $\mathrm{Y}$ in $\mathrm{G} / \mathrm{H}$ is multiplicity-free if $d(\mathrm{Y}, w)=1$ for all $w \in \mathrm{W}(\mathrm{Y})$. Equivalently, all oriented paths in $\Gamma(\mathrm{G} / \mathrm{H})$ with source $\mathrm{Y}$ contain only simple edges.

For example, $\mathrm{Y}$ is multiplicity-free if $r(\mathrm{Y})=r(\mathrm{G} / \mathrm{H})$, or if $\mathrm{H}$ is contained in a Borel subgroup of $\mathrm{G}$ (this follows from Lemma 5.)

Other examples of multiplicity-free orbit closures arise from parabolic induction: with notation as in Lemma 6, $\mathrm{Y}$ is multiplicity-free if and only if $\mathrm{Y}^{\prime}$ is.

If $\mathrm{G}$ is simply-laced, then $\mathrm{Y}$ is multiplicity-free if and only if it can be joined to $\mathrm{X}$ by an oriented path with only simple edges (Proposition 5).

Corollary 3. With notation as in Theorem 1, if $\mathrm{Y}$ is multiplicity-free then all irreducible components of $\mathrm{Y} \cap \mathrm{X}^{\prime}$ are multiplicity-free as well, and the corresponding intersection multiplicities equal 1. Moreover, for any $w \in \mathrm{W}(\mathrm{Y})$, the map $\varphi_{\mathrm{Y}, w}: \mathrm{S}_{\mathrm{Y}, w} \rightarrow \mathrm{S}$ is an isomorphism. As a consequence, $\mathrm{Y} \cap w^{-1} \mathrm{X}_{0}$ consists of smooth points of $\mathrm{Y}$.

Proof. The first assertion follows from Theorem 1. By Proposition $6, \varphi_{\mathrm{Y}, w}$ is 
finite surjective of degree 1, hence an isomorphism because $\mathrm{S}$ is smooth.

We next characterize those $\mathrm{B}$-orbit closures $\mathrm{Y} \in \mathcal{B}(\mathrm{G} / \mathrm{H}, \mathrm{X})$ that are multiplicity-free, in terms of the intersection numbers $\int_{\mathrm{X}}[\mathrm{Y}] \cdot\left[\mathrm{Y}^{\prime}\right]$ where $\mathrm{Y}^{\prime} \in \mathcal{B}(\mathrm{X})$. Here $\int_{\mathrm{X}}[\mathrm{Y}] \cdot\left[\mathrm{Y}^{\prime}\right]$ denotes the degree of the product of the classes of $\mathrm{Y}, \mathrm{Y}^{\prime}$ in the integral cohomology ring of $\mathrm{X}$; the latter is generated as an abelian group by classes of $\mathrm{B}$-stable subvarieties.

Corollary 4. With notation as in Theorem 1 , the numbers $\int_{\mathrm{X}}[\mathrm{Y}] \cdot\left[\mathrm{Y}^{\prime}\right]$ are 0 or powers of 2, for all $\mathrm{Y}^{\prime} \in \mathcal{B}(\mathrm{X})$. Moreover, $\mathrm{Y}$ is multiplicity-free if and only if $\int_{\mathrm{X}}[\mathrm{Y}] \cdot\left[\mathrm{Y}^{\prime}\right]$ equals 0 or 1 , for any $\mathrm{Y}^{\prime} \in \mathcal{B}(\mathrm{X})$.

Proof. Let $\mathrm{Y}^{\prime} \in \mathcal{B}(\mathrm{X})$. By [6] 1.4 Corollary, $\int_{\mathrm{X}}[\mathrm{Y}] \cdot\left[\mathrm{Y}^{\prime}\right] \neq 0$ if and only if: $\operatorname{dim}(\mathrm{Y})+\operatorname{dim}\left(\mathrm{Y}^{\prime}\right)=\operatorname{dim}(\mathrm{X})$, and $\mathrm{Y}$ meets $w_{0} \mathrm{Y}^{\prime}$ (where $w_{0}$ denotes the longest element of $\mathrm{W}$.) Under these hypotheses, $\mathrm{Y} \cap w_{0} \mathrm{Y}^{\prime}$ consists of a unique point $y$, fixed by $\mathrm{T}$. Moreover, the proof of [loc. cit.] shows that $w_{0} y \in \mathrm{Y}^{\prime 0}$. Thus, $\overline{\mathrm{B} y}$ and $\overline{\mathrm{B}^{-} y}=w_{0} \overline{\mathrm{B} w_{0} y}=w_{0} \mathrm{Y}^{\prime}$ meet transversally at $y$ in $\overline{\mathrm{G} y}=\mathrm{GY}^{\prime}$. As a consequence, we have

$\operatorname{dim}(\mathrm{B} y)=\operatorname{dim}\left(\mathrm{GY}^{\prime}\right)-\operatorname{dim}\left(w_{0} \mathrm{Y}^{\prime}\right)=\operatorname{dim}\left(\mathrm{GY}^{\prime}\right)+\operatorname{dim}(\mathrm{Y})-\operatorname{dim}(\mathrm{X})=\operatorname{dim}\left(\mathrm{Y} \cap \mathrm{GY}^{\prime}\right)$.

Since $\mathrm{Y} \cap \mathrm{GY}^{\prime}$ is equidimensional and $\mathrm{B}$-stable, it follows that its unique irreducible component through $y$ is $\overline{\mathrm{B} y}$.

Using the projection formula, we obtain

$$
\begin{aligned}
\int_{\mathrm{X}}[\mathrm{Y}] \cdot\left[\mathrm{Y}^{\prime}\right] & =\int_{\mathrm{X}}[\mathrm{Y}] \cdot\left[w_{0} \mathrm{Y}^{\prime}\right]=\int_{\mathrm{GY}^{\prime}}\left([\mathrm{Y}] \cdot\left[\mathrm{GY}^{\prime}\right]\right) \cdot\left[w_{0} \mathrm{Y}^{\prime}\right] \\
& =i\left(\overline{\mathrm{B} y}, \mathrm{Y} \cdot \mathrm{GY}^{\prime} ; \mathrm{X}\right) \int_{\mathrm{GY}^{\prime}} \overline{\mathrm{B} y} \cdot\left[w_{0} \mathrm{Y}^{\prime}\right]=i\left(\overline{\mathrm{B} y}, \mathrm{Y} \cdot \mathrm{GY}^{\prime} ; \mathrm{X}\right) .
\end{aligned}
$$

Thus, by Theorem $1, \int_{\mathrm{X}}[\mathrm{Y}] \cdot\left[\mathrm{Y}^{\prime}\right]$ is a power of 2 ; if moreover $\mathrm{Y}$ is multiplicity-free, then $\int_{\mathrm{X}}[\mathrm{Y}] \cdot\left[\mathrm{Y}^{\prime}\right]=1$.

Conversely, assume that $\int_{\mathrm{X}}[\mathrm{Y}] \cdot\left[\mathrm{Y}^{\prime}\right]$ equals 0 or 1 for all $\mathrm{Y}^{\prime} \in \mathcal{B}(\mathrm{X})$. Let then $w \in \mathrm{W}(\mathrm{Y})$; choose a closed $\mathrm{G}$-orbit $\mathrm{Z}$ with base point $z$ and consider $\mathrm{Y}^{\prime}=$ $\overline{\mathrm{B} w_{0} w^{-1} z}$. Then $w_{0} \mathrm{Y}^{\prime}$ is transversal to $\overline{\mathrm{B} w^{-1} z}$ at $z$ in $\mathrm{Z}$, so that $\operatorname{dim}\left(\mathrm{Y}^{\prime}\right)=$ $\operatorname{codim}_{\mathrm{Z}}\left(\overline{\mathrm{B} w^{-1} z}\right)$. But $\overline{\mathrm{B} w^{-1} z}$ is an irreducible component of $\mathrm{Y} \cap \mathrm{Z}$, and this intersection is proper. Thus, $\operatorname{dim}\left(\mathrm{Y}^{\prime}\right)=\operatorname{codim}_{\mathrm{X}}(\mathrm{Y})$. Since $\mathrm{Y}$ meets $w_{0} \mathrm{Y}^{\prime}$ at $w^{-1} z$, and $\mathrm{GY}^{\prime}=\mathrm{Z}$, we have

$$
\int_{\mathrm{X}}[\mathrm{Y}] \cdot\left[\mathrm{Y}^{\prime}\right]=i\left(\overline{\mathrm{B} w^{-1} z}, \mathrm{Y} \cdot \mathrm{Z} ; \mathrm{X}\right)
$$

and the latter equals $d(\mathrm{Y}, w)$, by [6] 1.4. It follows that $\mathrm{Y}$ is multiplicity-free.

We now show that the intersections of $\mathrm{B}$-orbit closures with $\mathrm{G}$-orbit closures in regular completions satisfy Hartshorne's connectedness theorem, see [11] 18.2. 
That theorem is proved there for schemes of depth at least 2 ; but B -orbit closures may have depth 1 at some points, see Example 6 in Section 4.

Theorem 2. Consider a regular completion $\mathrm{X}$ of $\mathrm{G} / \mathrm{H}$, a $\mathrm{B}$-orbit closure $\mathrm{Y}$ and a $\mathrm{G}$-orbit closure $\mathrm{X}^{\prime}$ in $\mathrm{X}$. Then $\mathrm{Y} \cap \mathrm{X}^{\prime}$ is connected in codimension 1 (that is, the complement in $\mathrm{Y} \cap \mathrm{X}^{\prime}$ of any closed subset of codimension at least 2 is connected.)

Proof. We may assume that $\mathrm{Y} \in \mathcal{B}(\mathrm{G} / \mathrm{H}, \mathrm{X})$. If $\mathrm{X}^{\prime}=\mathrm{Z}$ is a closed $\mathrm{G}$-orbit, then the irreducible components of $\mathrm{Y} \cap \mathrm{Z}$ are the $\overline{\mathrm{B} u} u^{-1} z$, where $u \in \mathrm{W}(\mathrm{Y})$. Consider neighbors $u, v \in \mathrm{W}(\mathrm{Y})$ and let $w \in \mathrm{W}$ be as in Proposition 3. Then $\overline{\mathrm{B} w^{-1} z}$ is a divisor in both $\overline{\mathrm{B} u^{-1} z}$ and $\overline{\mathrm{B} v^{-1} z}$. Since any two elements of $\mathrm{W}(\mathrm{Y})$ are connected by a chain of neighbors by Proposition 2, the assertion follows.

For arbitrary $\mathrm{X}^{\prime}$, let $\mathrm{Z}$ be a closed $\mathrm{G}$-orbit in $\mathrm{X}^{\prime}$. Let $\mathrm{Y}_{1}^{\prime}, \mathrm{Y}_{2}^{\prime}$ be unions of irreducible components of $\mathrm{Y} \cap \mathrm{X}^{\prime}$ such that $\mathrm{Y} \cap \mathrm{X}^{\prime}=\mathrm{Y}_{1}^{\prime} \cup \mathrm{Y}_{2}^{\prime}$. Then $\mathrm{Y}_{1}^{\prime} \cap \mathrm{Z}$ and $\mathrm{Y}_{2}^{\prime} \cap \mathrm{Z}$ are unions of irreducible components of $\mathrm{Y}^{\prime} \cap \mathrm{Z}$ (for any irreducible component of $\mathrm{Y} \cap \mathrm{X}^{\prime}$ meets $\mathrm{Z}$ properly in $\mathrm{X}^{\prime}$ ); Moreover, their intersection has codimension 1 in $\mathrm{Y}_{1}^{\prime} \cap \mathrm{Z}$ and $\mathrm{Y}_{2}^{\prime} \cap \mathrm{Z}$, by the first step of the proof. It follows that $\mathrm{Y}_{1}^{\prime} \cap \mathrm{Y}_{2}^{\prime}$ has codimension 1 in both $\mathrm{Y}_{1}^{\prime}$ and $\mathrm{Y}_{2}^{\prime}$.

\section{Orbit closures of maximal rank}

Let $\mathcal{B}(\mathrm{G} / \mathrm{H})_{\max }$, be the set of all $\mathrm{Y} \in \mathcal{B}(\mathrm{G} / \mathrm{H})$ such that $r(\mathrm{Y})=r(\mathrm{G} / \mathrm{H})$, that is, the set of all $\mathrm{B}$-orbit closures of maximal rank. Recall that all such orbit closures are multiplicity-free. Here is another characterization of them that generalizes a well known property of Schubert varieties.

Proposition 8. (i) For any $\mathrm{Y} \in \mathcal{B}(\mathrm{G} / \mathrm{H})_{\max }$, and $w \in \mathrm{W}(\mathrm{Y})$, we have: $\mathrm{B} w \mathrm{Y}^{0}=$ $(\mathrm{G} / \mathrm{H})^{0}$ and $w^{-1} \in \mathrm{W}^{\Delta(\mathrm{G} / \mathrm{H})}$. Moreover, $\mathrm{W}(\mathrm{Y})$ is disjoint from all $\mathrm{W}\left(\mathrm{Y}^{\prime}\right)$ where $\mathrm{Y}^{\prime} \in \mathcal{B}(\mathrm{G} / \mathrm{H})$ and $\mathrm{Y}^{\prime} \neq \mathrm{Y}$.

(ii) Conversely, if $\mathrm{Y} \in \mathcal{B}(\mathrm{G} / \mathrm{H})$ and there exists $w \in \mathrm{W}$ such that $\mathrm{B} w \mathrm{Y}^{0}=$ $(\mathrm{G} / \mathrm{H})^{0}$, then $\mathrm{Y}$ has maximal rank. If moreover $w^{-1} \in \mathrm{W}^{\Delta(\mathrm{G} / \mathrm{H})}$, then $w \in$ $\mathrm{W}(\mathrm{Y})$, and $\Delta(\mathrm{Y})$ consists of those $\alpha \in \Delta$ such that $w(\alpha) \in \Delta(\mathrm{G} / \mathrm{H})$.

Proof. (i) We prove that $\mathrm{B} w \mathrm{Y}^{0}=(\mathrm{G} / \mathrm{H})^{0}$ by induction over $\ell(w)$, the case where $\ell(w)=0$ being evident. If $\ell(w) \geq 1$, we can write $w=w^{\prime} s_{\alpha}$ for some simple root $\alpha$ and some $w^{\prime} \in \mathrm{W}$ such that $\ell\left(w^{\prime}\right)=\ell(w)-1$; then $\mathrm{B} w \mathrm{~B}=\mathrm{B} w^{\prime} \mathrm{B} s_{\alpha} \mathrm{B}$. Then $\mathrm{G} / \mathrm{H}=\overline{\mathrm{B} w \mathrm{Y}}=\overline{\mathrm{B} w^{\prime} \mathrm{P}_{\alpha} \mathrm{Y}}$. Since $\ell(w)=\operatorname{codim}_{\mathrm{G} / \mathrm{H}}(\mathrm{Y})$, it follows that $\alpha$ raises $\mathrm{Y}$ and that $w^{\prime} \in \mathrm{W}\left(\mathrm{P}_{\alpha} \mathrm{Y}\right)$. Because $\mathrm{Y}$ has maximal rank, $\mathrm{P}_{\alpha} \mathrm{Y}^{0}$ consists of two B-orbits, both of maximal rank. But $\mathrm{P}_{\alpha} \mathrm{Y}^{0}=\mathrm{Y}^{0} \cup \mathrm{B} s_{\alpha} \mathrm{Y}^{0}$ so that $\mathrm{B} s_{\alpha} \mathrm{Y}^{0}$ is a unique $\mathrm{B}$-orbit of maximal rank and of codimension $\ell\left(w^{\prime}\right)$ in $\mathrm{G} / \mathrm{H}$. By the induction assumption, we have $\mathrm{B} w^{\prime} \mathrm{B} s_{\alpha} \mathrm{Y}^{0}=(\mathrm{G} / \mathrm{H})^{0}$, that is, $\mathrm{B} w \mathrm{Y}^{0}=(\mathrm{G} / \mathrm{H})^{0}$. 
If moreover $w \in \mathrm{W}\left(\mathrm{Y}^{\prime}\right)$ for some $\mathrm{Y}^{\prime} \in \mathcal{B}(\mathrm{G} / \mathrm{H})$, then a similar induction shows that $\mathrm{Y}^{\prime}=\mathrm{Y}$.

If $w^{-1} \notin \mathrm{W}^{\Delta(\mathrm{G} / \mathrm{H})}$ then there exists $\beta \in \Delta(\mathrm{G} / \mathrm{H})$ such that $\ell\left(s_{\alpha} w\right)=\ell(w)-$ 1. Thus, $\mathrm{B} w \mathrm{~B}=\mathrm{B} s_{\beta} \mathrm{B} s_{\beta} w \mathrm{~B}$, so that $s_{\beta} \mathrm{B} s_{\beta} w \mathrm{Y}^{0}$ is contained in $(\mathrm{G} / \mathrm{H})^{0}$. But $s_{\beta}(\mathrm{G} / \mathrm{H})^{0}=(\mathrm{G} / \mathrm{H})^{0}$; therefore, $\mathrm{B} s_{\beta} w \mathrm{Y}^{0}=(\mathrm{G} / \mathrm{H})^{0}$, and $\overline{\mathrm{B} s_{\beta} w \mathrm{Y}}=\mathrm{G} / \mathrm{H}$. It follows that $\operatorname{codim}_{\mathrm{G} / \mathrm{H}}(\mathrm{Y}) \leq \ell\left(s_{\beta} w\right)=\ell(w)-1$, a contradiction.

(ii) Let $\dot{w}$ be a representative of $w$ in the normalizer of $\mathrm{T}$. By assumption, the map

$$
\begin{aligned}
\mathrm{U} \times \mathrm{Y}^{0} & \rightarrow(\mathrm{G} / \mathrm{H})^{0} \\
(u, y) & \mapsto u \dot{w} y
\end{aligned}
$$

is surjective. Thus, it induces an injective homomorphism from the ring $\mathbb{C}\left[(\mathrm{G} / \mathrm{H})^{0}\right]$ of regular functions on $(\mathrm{G} / \mathrm{H})^{0}$, to $\mathbb{C}\left[\mathrm{U} \times \mathrm{Y}^{0}\right]$. The group of invertible regular functions $\mathbb{C}\left[(\mathrm{G} / \mathrm{H})^{0}\right]^{*}$ is mapped into $\mathbb{C}\left[\mathrm{U} \times \mathrm{Y}^{0}\right]^{*}=\mathbb{C}\left[\mathrm{Y}^{0}\right]^{*}$. Quotienting by $\mathbb{C}^{*}$ and taking ranks, we obtain $r(\mathrm{G} / \mathrm{H}) \leq r(\mathrm{Y})$ by Lemma 1 , whence $r(\mathrm{Y})=$ $r(\mathrm{G} / \mathrm{H})$.

If moreover $w^{-1} \in \mathrm{W}^{\Delta(\mathrm{G} / \mathrm{H})}$, we show that $w \in \mathrm{W}(\mathrm{Y})$ by induction over $\ell(w)$; we may assume that $w \neq 1$. Then we can write $w=w^{\prime} s_{\alpha}$ where $w^{\prime} \in \mathrm{W}$, $\alpha \in \Delta$ and $\ell(w)=\ell\left(w^{\prime}\right)+1$. It follows that $w(\alpha) \in \Phi^{-}$.

We begin by checking that $s_{\alpha} \mathrm{Y}^{0} \neq \mathrm{Y}^{0}$. Otherwise, by Lemma 1 , there exists $y \in \mathrm{Y}^{0}$ fixed by $\left[\mathrm{L}_{\alpha}, \mathrm{L}_{\alpha}\right]$. Thus, $\dot{w} y \in(\mathrm{G} / \mathrm{H})^{0}$ is fixed by $w\left[\mathrm{~L}_{\alpha}, \mathrm{L}_{\alpha}\right] w^{-1}$. Since the unipotent radical of $\mathrm{P}(\mathrm{G} / \mathrm{H})$ acts freely on $(\mathrm{G} / \mathrm{H})^{0}$ by [17], it follows that $w(\alpha) \in \Phi_{\Delta(\mathrm{G} / \mathrm{H})}$. Then $\alpha \in \Delta \cap w^{-1}\left(\Phi_{\Delta(\mathrm{G} / \mathrm{H})}^{-}\right)$which contradicts the assumption that $w^{-1} \in \mathrm{W}^{\Delta(\mathrm{G} / \mathrm{H})}$.

As above, it follows that $\mathrm{B} s_{\alpha} \mathrm{Y}^{0}$ is a $\mathrm{B}$-orbit of maximal rank and of dimension $\operatorname{dim}(\mathrm{Y})+1$; moreover, $\mathrm{B} w^{\prime} \mathrm{B} s_{\alpha} \mathrm{Y}^{0}=(\mathrm{G} / \mathrm{H})^{0}$. We can write $w^{\prime}=$ $u v$ where $u \in \mathrm{W}_{\Delta(\mathrm{G} / \mathrm{H})}, v^{-1} \in \mathrm{W}^{\Delta(\mathrm{G} / \mathrm{H})}$, and $\ell\left(w^{\prime}\right)=\ell(u)+\ell(v)$. Thus, $\mathrm{B} w \mathrm{~B}=\mathrm{B} u \mathrm{~B} v \mathrm{~B} s_{\alpha} \mathrm{B}$, and $\mathrm{B} v \mathrm{~B} s_{\alpha} \mathrm{Y}^{0}=(\mathrm{G} / \mathrm{H})^{0}$ as $u^{-1}(\mathrm{G} / \mathrm{H})^{0}=(\mathrm{G} / \mathrm{H})^{0}$. By the induction assumption, $v \in \mathrm{W}\left(\overline{\mathrm{B} s_{\alpha} \mathrm{Y}}\right)$. Moreover, $\ell\left(v s_{\alpha}\right)=\ell(v)+1$, for $w=u v s_{\alpha}$ and $\ell(w)=\ell(u)+\ell(v)+1$. It follows that $v s_{\alpha} \in \mathrm{W}(\mathrm{Y})$; in particular, $s_{\alpha} v^{-1} \in \mathrm{W}^{\Delta(\mathrm{G} / \mathrm{H})}$. But $w^{-1}=s_{\alpha} v^{-1} u^{-1}$ is in $\mathrm{W}^{\Delta(\mathrm{G} / \mathrm{H})}$ as well. Thus, $u=1$ and $w^{-1} \in \mathrm{W}(\mathrm{Y})$.

Let $\alpha$ be a simple root of $\mathrm{Y}$. Then we see as above that $w(\alpha) \in \Phi_{\Delta(\mathrm{G} / \mathrm{H})}$. We have $w s_{\alpha}=s_{w(\alpha)} w$ with $s_{w(\alpha)} \in \mathrm{W}_{\Delta(\mathrm{G} / \mathrm{H})}$ and $w^{-1} \in \mathrm{W}^{\Delta(\mathrm{G} / \mathrm{H})}$. Thus, $\ell\left(w s_{\alpha}\right)=\ell\left(s_{w(\alpha)}\right)+\ell(w)$ which forces $w(\alpha) \in \Phi^{+}\left(\right.$as $\left.\ell\left(s_{\alpha} w\right)=\ell(w)+1\right)$ and $w(\alpha) \in \Delta\left(\right.$ as $\ell\left(s_{w(\alpha)}\right)=1$.) We conclude that $w(\alpha)$ is a simple root of $\mathrm{G} / \mathrm{H}$.

Conversely, let $\alpha \in \Delta$ such that $w(\alpha)$ is a simple root of $\mathrm{G} / \mathrm{H}$. Then $\ell\left(w s_{\alpha}\right)=\ell(w)+1$, whence

$\mathrm{B} w \mathrm{~B} s_{\alpha} \mathrm{Y}^{0}=\mathrm{B} w s_{\alpha} \mathrm{Y}^{0}=\mathrm{B} s_{w(\alpha)} w \mathrm{Y}^{0}=\mathrm{B} s_{w(\alpha)} \mathrm{B} w \mathrm{Y}^{0}=\mathrm{B} s_{w(\alpha)}(\mathrm{G} / \mathrm{H})^{0}=(\mathrm{G} / \mathrm{H})^{0}$.

Let $\mathcal{O}$ be a $\mathrm{B}$-orbit in $\mathrm{B} s_{\alpha} \mathrm{Y}^{0}$. Then $\mathrm{B} w \mathcal{O}=(\mathrm{G} / \mathrm{H})^{0}$. By $(\mathrm{i})$, we have $\mathcal{O}=\mathrm{Y}^{0}$, whence $s_{\alpha} \mathrm{Y}^{0}=\mathrm{Y}^{0}$ and $\alpha \in \Delta(\mathrm{Y})$. 
This preliminary result, combined with those of Section 2, implies a structure theorem for orbit closures of maximal rank in a regular completion $\mathrm{X}$. We denote by $\mathcal{B}(\mathrm{X})_{\max }$ the set of these orbit closures, a subset of $\mathcal{B}(\mathrm{G} / \mathrm{H}, \mathrm{X})$.

Theorem 3. Let $\mathrm{Y} \in \mathcal{B}(\mathrm{X})_{\max }$ and $w \in \mathrm{W}(\mathrm{Y})$. Choose a "slice" $\mathrm{S}_{\mathrm{Y}, w}$ as in Proposition 6, so that the product map

$$
\left(\mathrm{U} \cap w^{-1} \mathrm{R}_{u}(\mathrm{P}) w\right) \times w^{-1} \mathrm{~S}_{\mathrm{Y}, w} \rightarrow \mathrm{Y} \cap w^{-1} \mathrm{X}_{0}
$$

is an isomorphism. Then $w^{-1} \mathrm{~S}_{\mathrm{Y}, w}$ is fixed pointwise by $[\mathrm{L}(\mathrm{Y}), \mathrm{L}(\mathrm{Y})]$. Moreover, $\mathrm{Y} \cap w^{-1} \mathrm{X}_{0}$ is $\mathrm{P}(\mathrm{Y})$-stable and meets each $\mathrm{G}$-orbit along a unique $\mathrm{B}$ orbit, of maximal rank in this $\mathrm{G}$-orbit. In particular, there exists $y \in \mathrm{Y}^{0}$ fixed by $[\mathrm{L}(\mathrm{Y}), \mathrm{L}(\mathrm{Y})]$ such that the product map $\left(\mathrm{U} \cap w^{-1} \mathrm{R}_{u}(\mathrm{P}) w\right) \times \mathrm{T} y \rightarrow \mathrm{Y}^{0}$ is an isomorphism.

As a consequence, for each $\mathrm{G}$-orbit closure $\mathrm{X}^{\prime}$ in $\mathrm{X}$, all irreducible components of $\mathrm{Y} \cap \mathrm{X}^{\prime}$ have maximal rank in $\mathrm{X}^{\prime}$. Moreover, a given $\mathrm{Y}^{\prime} \in \mathcal{B}\left(\mathrm{X}^{\prime}\right)$ is an irreducible component of $\mathrm{Y} \cap \mathrm{X}^{\prime}$ if and only if $\mathrm{W}\left(\mathrm{Y}^{\prime}\right)$ is contained in $\mathrm{W}(\mathrm{Y})$.

Proof. With notation as in Section 2, recall that

$$
w^{-1} \mathrm{~S}_{\mathrm{Y}, w}=\mathrm{Y} \cap\left(\mathrm{U}^{-} \cap w^{-1} \mathrm{U} w\right) w^{-1} \mathrm{~S}
$$

and that $[\mathrm{L}(\mathrm{X}), \mathrm{L}(\mathrm{X})]$ acts trivially on $\mathrm{S}$. Together with Proposition 8, it follows that $[\mathrm{L}(\mathrm{Y}), \mathrm{L}(\mathrm{Y})]$ fixes pointwise $\mathrm{S}$ and normalizes $\mathrm{U}^{-} \cap w^{-1} \mathrm{U} w$. Thus, $[\mathrm{L}(\mathrm{Y}), \mathrm{L}(\mathrm{Y})]$ stabilizes $w^{-1} \mathrm{~S}_{\mathrm{Y}, w}$. Moreover, intersecting that space with those boundary divisors that contain a given closed $\mathrm{G}$-orbit, we obtain $[\mathrm{L}(\mathrm{Y}), \mathrm{L}(\mathrm{Y})]$ stable hypersurfaces meeting transversally at a fixed point. Therefore, $[\mathrm{L}(\mathrm{Y}), \mathrm{L}(\mathrm{Y})]$ fixes pointwise $w^{-1} \mathrm{~S}_{Y, w}$.

By Proposition $6, w^{-1} \mathrm{~S}_{\mathrm{Y}, w}$ meets each $\mathrm{G}$-orbit along a unique $\mathrm{T}$-orbit. As a consequence, the intersection of $\mathrm{Y} \cap w^{-1} \mathrm{X}_{0}$ with each $\mathrm{G}$-orbit is contained in a unique $\mathrm{B}$-orbit. We apply this to $\mathrm{GY}^{0}$, the open $\mathrm{G}$-orbit in $\mathrm{X}$. Since $\mathrm{Y} \cap w^{-1} \mathrm{X}_{0} \cap \mathrm{G} \mathrm{Y}^{0}=\mathrm{Y} \cap w^{-1} \mathrm{X}^{0}$ equals $\mathrm{Y}^{0}$ by Proposition 8, we see that the product map

$$
\left(\mathrm{U} \cap w^{-1} \mathrm{R}_{u}(\mathrm{P}) w\right) \times\left(w^{-1} \mathrm{~S}_{\mathrm{Y}, w} \cap \mathrm{Y}^{0}\right) \rightarrow \mathrm{Y}^{0}
$$

is an isomorphism. Moreover, $w^{-1} \mathrm{~S}_{\mathrm{Y}, w} \cap \mathrm{Y}^{0}$ is a unique $\mathrm{T}$-orbit of dimension equal to the rank of $\mathrm{X}$.

It follows that each $\mathrm{U}$-orbit in $\mathrm{Y}^{0}$ is a unique orbit of $\mathrm{U} \cap w^{-1} \mathrm{R}_{u}(\mathrm{P}) w$. Indeed, any $\mathrm{U}$-orbit is isomorphic to some affine space, and its projection to $w^{-1} \mathrm{~S}_{\mathrm{Y}, w} \cap \mathrm{Y}^{0}$ is a morphism to a torus, hence is constant.

Choose $y_{0} \in \mathrm{Y}^{0}$ and let $y \in \mathrm{Y} \cap w^{-1} \mathrm{X}_{0}$. Since $\mathrm{B} y_{0}=\mathrm{Y}^{0}$ is dense in $\mathrm{Y} \cap$ $w^{-1} \mathrm{X}_{0}$, we have $\operatorname{dim}(\mathrm{U} y) \leq \operatorname{dim}\left(\mathrm{U} y_{0}\right)$. The latter equals $\operatorname{dim}\left(\mathrm{U} \cap w^{-1} \mathrm{R}_{u}(\mathrm{P}) w\right)$ by the previous step. Because $\mathrm{U} \cap w^{-1} \mathrm{R}_{u}(\mathrm{P}) w$ acts freely on $\mathrm{Y} \cap w^{-1} \mathrm{X}_{0}$, it follows that $\left(\mathrm{U} \cap w^{-1} \mathrm{R}_{u}(\mathrm{P}) w\right) y$ is open in $\mathrm{U} y$. But both are affine spaces, so that they are equal. Thus, $\mathrm{Y} \cap w^{-1} \mathrm{X}_{0}$ is $\mathrm{B}$-stable. It is even $\mathrm{P}(\mathrm{Y})$-stable, because $\mathrm{P}(\mathrm{Y}) \subseteq w^{-1} \mathrm{P} w$ by Proposition 8 . 
Since $w^{-1} \mathrm{~S}_{\mathrm{Y}, w}$ meets each $\mathrm{G}$-orbit along a unique $\mathrm{T}$-orbit, $\mathrm{Y} \cap w^{-1} \mathrm{X}_{0}$ meets each $\mathrm{G}$-orbit along a unique $\mathrm{B}$-orbit. Let $y \in \mathrm{Y} \cap w^{-1} \mathrm{X}_{0}$, then $w \mathrm{~B} y \subseteq \mathrm{X}_{0}$ and, therefore, $w \mathrm{~B} y \subseteq(\mathrm{G} y)^{0}$. By Proposition 8 again, we have $r(\mathrm{~B} y)=r(\mathrm{G} y)$.

The remaining assertions follow from Theorem 1 together with Proposition 8.

As a consequence, we determine all $\mathrm{B}$-orbit closures $\mathrm{Y}^{\prime}$ such that $\int_{\mathrm{X}}[\mathrm{Y}] \cdot\left[\mathrm{Y}^{\prime}\right] \neq$ 0 ; by Corollary 4 , this amounts to $\int_{\mathrm{X}}[\mathrm{Y}] \cdot\left[\mathrm{Y}^{\prime}\right]=1$.

Corollary 5. Let $\mathrm{Y}$ be a $\mathrm{B}$-orbit closure of maximal rank in a regular completion $\mathrm{X}$ and let $\mathrm{Y}^{\prime} \in \mathcal{B}(\mathrm{X})$. Then the intersection number $\int_{\mathrm{X}}[\mathrm{Y}] \cdot\left[\mathrm{Y}^{\prime}\right]$ equals 1 if and only if $\mathrm{Y}^{\prime}=\overline{\mathrm{B} w_{0} w^{-1} z}$ for some $w \in \mathrm{W}(\mathrm{Y})$ and some closed $\mathrm{G}$-orbit $\mathrm{Z}$ with base point $z$.

Proof. If $\int_{\mathrm{X}}[\mathrm{Y}] \cdot\left[\mathrm{Y}^{\prime}\right]=1$, then $\mathrm{Y} \cap w_{0} \mathrm{Y}^{\prime}$ consists of a unique $\mathrm{T}$-fixed point $y \in w_{0} \mathrm{Y}^{\prime 0}$, and $\overline{\mathrm{B} y}$ is an irreducible component of $\mathrm{Y} \cap \mathrm{GY}^{\prime}$, by the proof of Corollary 4. Therefore, $\overline{\mathrm{B} y}$ has maximal rank in $\mathrm{GY}^{\prime}=\overline{\mathrm{G} y^{\prime}}$. But $r(\overline{\mathrm{B} y})=0$ because $y$ is fixed by $\mathrm{T}$. Thus $\mathrm{G} y$, being a $\mathrm{G}$-orbit of rank 0 , is closed in $\mathrm{X}$. Let $z$ be its base point, then $y=w^{-1} z$ for some $w \in \mathrm{W}(\mathrm{Y})$, so that $\mathrm{Y}^{\prime}=\overline{\mathrm{B} w_{0} y}=\overline{\mathrm{B} w_{0} w^{-1} z}$. The converse is clear.

We now describe the intersections of $\mathrm{B}$-orbit closures of maximal rank with $\mathrm{G}$-orbit closures, in terms of Knop's action of the Weyl group $\mathrm{W}$ on the set $\mathcal{B}(\mathrm{X})$. This action can be defined as follows.

Let $\alpha \in \Delta$ and $\mathrm{Y} \in \mathcal{B}(\mathrm{X})$, then $s_{\alpha}$ fixes $\mathrm{Y}$, except in the following cases:

- Type $\mathrm{U}: \mathrm{P}_{\alpha} \mathrm{Y}^{0}=\mathrm{Y}^{0} \cup \mathrm{Z}^{0}$ for $\mathrm{Z} \in \mathcal{B}(\mathrm{X})$ with $r(\mathrm{Z})=r(\mathrm{Y})$. Then $s_{\alpha}$ exchanges $\mathrm{Y}$ and $\mathrm{Z}$.

- Type $\mathrm{T}: \mathrm{P}_{\alpha} \mathrm{Y}^{0}=\mathrm{Y}^{0} \cup \mathrm{Y}_{-}^{0} \cup \mathrm{Z}^{0}$ for $\mathrm{Z} \in \mathcal{B}(\mathrm{X})$ with $r(\mathrm{Y})=r\left(\mathrm{Y}_{-}\right)=r(\mathrm{Z})-1$. Then $s_{\alpha}$ exchanges $\mathrm{Y}$ and $\mathrm{Y}_{-}$.

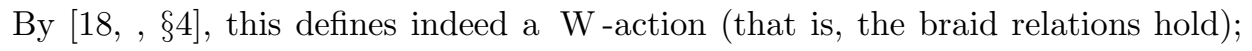
moreover, $X(w(\mathrm{Y}))=w(X(\mathrm{Y}))$ for all $w \in \mathrm{W}$. In particular, this action preserves the rank (and, obviously, the subset $\mathcal{B}(\mathrm{G} / \mathrm{H}, \mathrm{X}$ ). If moreover $\mathrm{G}$ is simplylaced, then this action preserves multiplicity-freeness by Proposition 5; but this does not extend to multiply-laced $\mathrm{G}$, as shown by Example 4.

For $\mathrm{Y} \in \mathcal{B}(\mathrm{X})_{\max }$ and $w \in \mathrm{W}(\mathrm{Y})$, we have $w(\mathrm{Y})=\mathrm{X}$. Thus, $\mathcal{B}(\mathrm{X})_{\max }$ is the $\mathrm{W}$-orbit of $\mathrm{X}$ in $\mathcal{B}(\mathrm{X})$. The isotropy group of $\mathrm{X}$ depends only on $\mathrm{G} / \mathrm{H}$; denote it by $\mathrm{W}(\mathrm{G} / \mathrm{H})$. This group acts on $X(\mathrm{G} / \mathrm{H})$ and contains $\mathrm{W}_{\Delta(\mathrm{G} / \mathrm{H})}$. The latter acts trivially on $X(\mathrm{G} / \mathrm{H})$ by Lemma 1 . In fact, $\mathrm{W}_{(\mathrm{G} / \mathrm{H})}$ stabilizes $\Phi_{\Delta(\mathrm{G} / \mathrm{H})}$ (indeed, $\Phi_{\Delta(\mathrm{G} / \mathrm{H})}$ consists of all roots that are orthogonal to $X(\mathrm{G} / \mathrm{H})$, if $\mathrm{G} / \mathrm{H}$ is non-degenerate in the sense of [17]; and the general case reduces to that one, by [17] §5.)

The normalizer of $\Phi_{\Delta(\mathrm{G} / \mathrm{H})}$ in $\mathrm{W}$ is the semi-direct product of $\mathrm{W}_{\Delta(\mathrm{G} / \mathrm{H})}$ with the normalizer of $\Delta(\mathrm{G} / \mathrm{H})$. Therefore, $\mathrm{W}_{(\mathrm{G} / \mathrm{H})}$ is the semi-direct product 
of $\mathrm{W}_{\Delta(\mathrm{G} / \mathrm{H})}$ with

$$
\mathrm{W}_{\mathrm{G} / \mathrm{H}}=\{w \in \mathrm{W} \mid w(\mathrm{G} / \mathrm{H})=\mathrm{G} / \mathrm{H} \quad \text { and } \quad w(\Delta(\mathrm{G} / \mathrm{H}))=\Delta(\mathrm{G} / \mathrm{H})\} .
$$

The latter identifies to the image of $\mathrm{W}_{(\mathrm{G} / \mathrm{H})}$ in $\operatorname{Aut} X(\mathrm{G} / \mathrm{H})$, that is, to the "Weyl group of $\mathrm{G} / \mathrm{H}$ ", see [18] Theorem 6.2.

In fact, $\mathrm{W}_{\mathrm{G} / \mathrm{H}}$ is the set of all $w \in \mathrm{W}_{(\mathrm{G} / \mathrm{H})}$ such that $w(\rho)-\rho \in X(\mathrm{G} / \mathrm{H})$, where $\rho$ denotes the half sum of positive roots (see [16] 6.5); we shall not need this result.

Let

$$
\mathrm{W}^{(\mathrm{G} / \mathrm{H})}=\left\{w \in \mathrm{W} \mid \ell(w u) \geq \ell(w) \quad \forall u \in \mathrm{W}_{(\mathrm{G} / \mathrm{H})}\right\},
$$

the set of all elements of minimal length in their right $\mathrm{W}_{(\mathrm{G} / \mathrm{H})}$-coset.

Proposition 9. With notation as above, we have

$$
\mathrm{W}^{(\mathrm{G} / \mathrm{H})}=\left\{w \in \mathrm{W}^{\Delta(\mathrm{G} / \mathrm{H})} \mid \ell(w u) \geq \ell(w) \quad \forall u \in \mathrm{W}_{(\mathrm{G} / \mathrm{H})}\right\}
$$

and, for any $w \in \mathrm{W}$,

$$
\mathrm{W}(w(\mathrm{G} / \mathrm{H}))=\left\{v \in \mathrm{W} \mid v^{-1} \in \mathrm{W}^{(\mathrm{G} / \mathrm{H})} \cap w \mathrm{~W}_{(\mathrm{G} / \mathrm{H})}\right\} .
$$

As a consequence, all elements of minimal length in a given left $\mathrm{W}_{(\mathrm{G} / \mathrm{H})}$ -

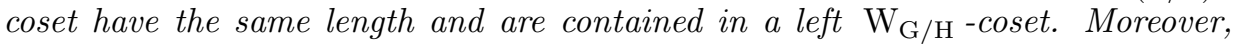
the subsets $\mathrm{W}(\mathrm{Y}), \mathrm{Y} \in \mathcal{B}(\mathrm{G} / \mathrm{H})_{\max }$, are exactly the subsets of all elements of minimal length in a given left $\mathrm{W}_{(\mathrm{G} / \mathrm{H})}$-coset.

For any $\mathrm{G}$-orbit closure $\mathrm{X}^{\prime}$ in a regular completion $\mathrm{X}$, we have

$$
w(\mathrm{X}) \cap \mathrm{X}^{\prime}=\bigcup_{w^{\prime} \in \mathrm{W}^{(\mathrm{G} / \mathrm{H})} \cap w \mathrm{~W}_{(\mathrm{G} / \mathrm{H})}} w^{\prime}\left(\mathrm{X}^{\prime}\right) .
$$

Proof. Clearly, $\mathrm{W}^{(\mathrm{G} / \mathrm{H})}$ is contained in $\mathrm{W}^{\Delta(\mathrm{G} / \mathrm{H})}$. And since $\mathrm{W}_{\mathrm{G} / \mathrm{H}}$ stabilizes $\Delta(\mathrm{G} / \mathrm{H})$, the set $\mathrm{W}^{\Delta(\mathrm{G} / \mathrm{H})}$ is stable under right multiplication by $\mathrm{W}_{\mathrm{G} / \mathrm{H}}$. This implies the first assertion.

Let $\mathrm{Y}=w(\mathrm{G} / \mathrm{H})$ and observe that $\operatorname{codim}_{\mathrm{G} / \mathrm{H}}(\mathrm{Y}) \leq \ell(w)$ with equality if and only if $w^{-1} \in \mathrm{W}(\mathrm{Y})$ (indeed, a reduced decomposition of $w$ defines a non-oriented path in $\Gamma(\mathrm{G} / \mathrm{H})$ with endpoints $\mathrm{Y}$ and $\mathrm{G} / \mathrm{H})$.

Let $v \in \mathrm{W}(\mathrm{Y})$. Since $v(\mathrm{Y})=\mathrm{G} / \mathrm{H}$, we have $v^{-1} \in w \mathrm{~W}_{(\mathrm{G} / \mathrm{H})}$. Moreover, $\ell\left(v^{-1}\right)=\ell(v)=\operatorname{codim}_{\mathrm{X}}(\mathrm{Y}) \leq \ell(w)$. Since we can change $w$ in its right $\mathrm{W}_{(\mathrm{X})}$ coset, it follows that $v^{-1} \in \mathrm{W}^{(\mathrm{G} / \mathrm{H})}$.

Conversely, let $u \in \mathrm{W}$ such that $u^{-1} \in \mathrm{W}^{(\mathrm{G} / \mathrm{H})} \cap w \mathrm{~W}_{(\mathrm{G} / \mathrm{H})}$. Then $u(\mathrm{Y})=$ $\mathrm{G} / \mathrm{H}$, whence $\ell(u) \geq \ell(v)$ and $u \in \mathrm{W}_{(\mathrm{G} / \mathrm{H})} v$. Since $u^{-1} \in \mathrm{W}^{(\mathrm{G} / \mathrm{H})}$, this forces $\ell(u)=\ell(v)$ and then $u \in \mathrm{W}(\mathrm{Y})$. This proves the first assertion. Together with Theorem 3, this implies the second assertion.

Example 5. Let $\mathbf{G}$ be a connected reductive group. Consider the group $G=$ $\mathbf{G} \times \mathbf{G}$ acting on $\mathbf{G}$ by $(x, y) \cdot z=x z y^{-1}$. Then $\mathbf{G}$ is a spherical homogeneous 
space: consider the Borel subgroup $B=\mathbf{B} \times \mathbf{B}^{-}$of $\mathbf{G}$, where $\mathbf{B}$ and $\mathbf{B}^{-}$are opposed Borel subgroups of $\mathbf{G}$. With evident notation, the $\mathbf{B}$-orbits in $\mathbf{G}$ are the $\mathbf{B} w \mathbf{B}^{-}, w \in \mathbf{W}$. This identifies $\mathcal{B}(\mathbf{G})$ to $\mathbf{W}$. Morever, all $\mathbf{B}$-orbits have maximal rank, and the Weyl group $\mathbf{W}=\mathbf{W} \times \mathbf{W}$ acts on $\mathbf{W}$ by $(u, v) w=u w v^{-1}$. Thus, $\Delta(\mathbf{G})$ is empty, $\mathrm{W}_{(\mathrm{G})}$ is the diagonal in $\mathbf{W} \times \mathbf{W}$, and $\mathbf{W} \times\{1\}$ is a system of representatives of $\mathrm{W} / \mathrm{W}_{(\mathbf{G})}$. One checks that

$$
\mathrm{W}^{(\mathbf{G})}=\left\{(u, v) \in \mathbf{W} \times \mathbf{W} \mid \ell(u)+\ell(v)=\ell\left(u v^{-1}\right)\right\} .
$$

In particular, $(w, 1) \in \mathbf{W}^{(\mathbf{G})}$ for all $w \in \mathbf{W}$. Moreover,

$$
\mathrm{W}^{(\mathbf{G})} \cap(w, 1) \mathrm{W}_{(\mathbf{G})}=\left\{(u, v) \in \mathbf{W} \times \mathbf{W} \mid u v^{-1}=w \quad \text { and } \quad \ell(u)+\ell(v)=\ell(w)\right\} .
$$

This identifies $\mathbf{W}^{(\mathbf{G})} \cap(w, 1) \mathrm{W}_{(\mathbf{G})}$ to the set of all $u \in \mathbf{W}$ such that $u \preceq w$ for the right weak order on $\mathbf{W}$. Now the last statement of Proposition 9 gives back [6] Theorem 2.1.

Returning to arbitrary G/H, we shall deduce from Proposition 4 the following

Theorem 4. The group $\mathrm{W}_{(\mathrm{G} / \mathrm{H})}$ is generated by reflections $s_{\alpha}$ where $\alpha$ is a root such that $\alpha \in \Phi_{\Delta(\mathrm{G} / \mathrm{H})}$ or that $2 \alpha \in X(\mathrm{G} / \mathrm{H})$, and by products $s_{\alpha} s_{\beta}$ where $\alpha, \beta$ are orthogonal roots such that $\alpha+\beta \in X(\mathrm{G} / \mathrm{H})$.

Proof. Let $w \in \mathrm{W}_{(\mathrm{G} / \mathrm{H})}$. We choose a reduced decomposition $w=s_{\alpha_{\ell}} \cdots s_{\alpha_{2}} s_{\alpha_{1}}$ and we argue by induction on the length $\ell$.

If $\alpha_{1} \in \Delta(\mathrm{G} / \mathrm{H})$ then $s_{\alpha_{1}}$ is a reflection in $\mathrm{W}_{(\mathrm{G} / \mathrm{H})}$, so that $s_{\alpha_{\ell}} \cdots s_{\alpha_{2}} \in$ $\mathrm{W}_{(\mathrm{G} / \mathrm{H})}$. Now we conclude by the induction assumption.

If $\alpha_{1} \notin \Delta(\mathrm{G} / \mathrm{H})$ then $s_{\alpha_{1}}(\mathrm{G} / \mathrm{H})$ has codimension 1 in $\mathrm{G} / \mathrm{H}$. Let $i$ be the largest integer such that $\operatorname{codim}_{\mathrm{G} / \mathrm{H}} s_{\alpha_{i}} \cdots s_{\alpha_{1}}(\mathrm{G} / \mathrm{H})=i$. Let $\mathrm{Y}=s_{\alpha_{i}} \cdots s_{\alpha_{1}}$ $(\mathrm{G} / \mathrm{H})=i$, then $\mathrm{Y} \in \mathcal{B}(\mathrm{G} / \mathrm{H})_{\max }$ and $s_{\alpha_{1}} \cdots s_{\alpha_{1}} \in \mathrm{W}(\mathrm{Y})$.

If $\mathrm{P}_{\alpha_{i+1}} \mathrm{Y}=\mathrm{Y}$ then $s_{\alpha_{i+1}}(\mathrm{Y})=\mathrm{Y}$ by definition of the $\mathrm{W}$-action and maximality of $i$. Let $\alpha=s_{\alpha_{1}} \cdots s_{\alpha_{i}}\left(\alpha_{i+1}\right)$. Then $s_{\alpha}$ is a reflection of $\mathrm{W}_{(\mathrm{G} / \mathrm{H})}$, and $w=s_{\alpha_{\ell}} \cdots s_{\alpha_{i+2}} s_{\alpha_{i}} \cdots s_{\alpha_{1}} s_{\alpha}$. If $\alpha_{i+1} \in \Delta(\mathrm{Y})$, then $\alpha \in \Delta(\mathrm{G} / \mathrm{H})$ by Proposition 8. Otherwise, $\mathrm{P}_{\alpha_{i+1}} \mathrm{Y}^{0} / \mathrm{R}\left(\mathrm{P}_{\alpha_{i+1}}\right)$ is isomorphic to $\mathrm{PGL}(2) / \mathrm{T}$ or to PGL $(2) / \mathrm{N}$; it follows that $2 \alpha_{i+1} \in X(\mathrm{Y})$, and that $2 \alpha \in X(\mathrm{G} / \mathrm{H})$. Now we conclude by the induction assumption.

If $\mathrm{P}_{\alpha_{i+1}} \mathrm{Y} \neq \mathrm{Y}$ then $\alpha_{i+1}$ raises $\mathrm{Y}$ to (say) $\mathrm{Y}^{\prime}$. Choose $u \in \mathrm{W}\left(\mathrm{Y}^{\prime}\right)$, then $\ell(u)=i-1$ and $u s_{\alpha_{i+1}} \in \mathrm{W}(\mathrm{Y})$. Moreover, $u s_{\alpha_{i+1}} s_{\alpha_{i}} \cdots s_{\alpha_{1}} \in \mathrm{W}_{(\mathrm{G} / \mathrm{H})}$. We have $w=v_{u s} s_{\alpha_{i+1}} s_{\alpha_{i}} \cdots s_{\alpha_{1}}$ for some $v \in \mathrm{W}_{(\mathrm{G} / \mathrm{H})}$ such that $\ell(v u)=\ell-i-1$. Thus, $\ell(v) \leq \ell(v u)+\ell(u)=\ell-2$. Therefore, we may assume that there exist $\mathrm{Y} \in \mathcal{B}(\mathrm{G} / \mathrm{H})_{\max }$ and $w_{1}, w_{2} \in \mathrm{W}(\mathrm{Y})$ such that $w=w_{2} w_{1}^{-1}$. By Proposition 2, we may assume moreover that $w_{1}$ and $w_{2}$ are neighbors. Then we conclude by Proposition 4.

As a direct consequence, we recover the following result of Knop, see [17] and [18]. 
Corollary 6. The image of $\mathrm{W}_{\mathrm{G} / \mathrm{H}}$ in Aut $\mathcal{X}(\mathrm{G} / \mathrm{H})$ is generated by reflections.

\section{Singularities of orbit closures}

We begin by recalling the notion of rational singularities, see e.g. [14] p. 50.

Let $\mathrm{Y}$ be a variety. Choose a resolution of singularities $\varphi: \mathrm{Z} \rightarrow \mathrm{Y}$, that is, $\mathrm{Z}$ is smooth and $\varphi$ is proper and birational. Then the sheaves $\mathrm{R}^{n} \varphi_{*} \mathcal{O}_{\mathrm{Z}}$ $(n \geq 0)$ are independent of the choice of $\mathrm{Z}$. The singularities of $\mathrm{Y}$ are rational if $\mathrm{R}^{n} \varphi_{*} \mathcal{O}_{\mathrm{Z}}=0$ for all $n \geq 1$ and $\varphi_{*} \mathcal{O}_{\mathrm{Z}}=\mathcal{O}_{\mathrm{Y}}$; the latter condition is equivalent to normality of $\mathrm{Y}$. Varieties with rational singularities are Cohen-Macaulay.

Let now $\mathrm{X}$ be a spherical variety and $\mathrm{Y}$ a $\mathrm{B}$-stable subvariety. If $\mathrm{Y}$ is $\mathrm{G}$ stable, then its singularities are rational, see e.g. [5]. But this does not extend to arbitrary Y : generalizing Example 1 in Section 1, we shall construct examples of $\mathrm{B}$-orbit closures in a spherical homogeneous space, of arbitrary dimension but of depth 1 at some points. In particular, such orbit closures are neither normal nor Cohen-Macaulay.

Example 6. Let $r, n$ be integers such that $0 \leq r \leq n-1$. Let $\mathrm{X}$ be the space of smooth quadrics of dimension $r$ in projective space $\mathbb{P}^{n}$ : points of $\mathrm{X}$ are pairs $(\mathrm{Q}, \mathrm{L})$ where $\mathrm{L}$ is a linear subspace of $\mathbb{P}^{n}$ of dimension $r+1$, and $\mathrm{Q}$ is a smooth hypersurface of degree 2 in $\mathrm{L}$. The group $\mathrm{G}=\mathrm{GL}(n+1)$ acts transitively on $\mathrm{X}$; an isotropy group $\mathrm{H}$ consists of all matrices of the form $\left(\begin{array}{cc}\mathrm{A} & \mathrm{B} \\ 0 & \mathrm{C}\end{array}\right)$ where $\mathrm{A}$ is a scalar multiple of an orthogonal $(r+1) \times(r+1)$ matrix, $\mathrm{B}$ is a $(r+1) \times n$ matrix, and $\mathrm{C}$ is an invertible $(n-r) \times(n-r)$ matrix. Thus, $\mathrm{X}$ is obtained from the symmetric space $\mathrm{GL}(r+1) / \mathrm{O}(r+1) \mathbb{C}^{*}$ by parabolic induction. It follows that $\mathrm{X}$ is spherical of rank $r+1$.

Let $m$ be an integer such that $1 \leq m \leq n-r-1$ and let $\mathbb{P}^{m}$ be a linear subspace of $\mathbb{P}^{n}$ of dimension $m$. Consider the set

$$
\mathrm{Y}_{m}=\left\{(\mathrm{Q}, \mathrm{L}) \in \mathrm{X} \mid \mathrm{Q} \text { meets } \mathbb{P}^{m}\right\}
$$

a subvariety of $\mathrm{X}$ of codimension $n-m-r$. The stabilizer $\mathrm{P}_{m}$ of $\mathbb{P}^{m}$ in $\mathrm{G}$, a maximal parabolic subgroup, stabilizes $\mathrm{Y}_{m}$ as well; in fact, $\mathrm{Y}_{m}$ contains an open $\mathrm{P}_{m}$-orbit, the subset of all $(\mathrm{Q}, \mathrm{L})$ such that $\mathrm{Q}$ meets $\mathbb{P}^{m}$ in a unique point. In particular, $\mathrm{Y}_{m}$ is an orbit closure of a Borel subgroup of $\mathrm{G}$; one checks that $r\left(\mathrm{Y}_{m}\right)=r$. The corresponding $\mathrm{H}$-orbit closure $\mathrm{V}_{m}$ in the flag variety of $\mathrm{G}$ is the set of all complete flags whose $m$-dimensional subspace meets the $\mathrm{H}$ fixed quadric. Thus, $\mathrm{V}_{m}$ is irreducible for $r \geq 1$, whereas it has two irreducible components for $r=0$ (then the quadric consists of two points).

We claim that the non-normal locus of $\mathrm{Y}_{m}$ equals the complement $\mathrm{Y}_{m}^{\prime}$ of its open $\mathrm{P}_{m}$-orbit. For this, note that the set of all $(\mathrm{Q}, \mathrm{L})$ in $\mathrm{X}$ such that $\mathrm{Q}$ meets 
$\mathbb{P}^{m}$ in two distinct points is a unique $\mathrm{P}_{m}$-orbit, dense in $\mathrm{Y}_{m}^{\prime}$. Let

$$
\tilde{\mathrm{Y}}_{m}=\left\{(x, \mathrm{Q}, \mathrm{L}) \in \mathbb{P}^{n} \times \mathrm{X} \mid x \in \mathrm{Q} \cap \mathbb{P}^{m}\right\}
$$

Then $\tilde{\mathrm{Y}}_{m}$, is a $\mathrm{P}_{m}$-variety, and the map $\nu: \tilde{\mathrm{Y}}_{m} \rightarrow \mathrm{Y}_{m},(x, \mathrm{Q}, \mathrm{L}) \mapsto(\mathrm{Q}, \mathrm{L})$ is an isomorphism over the open $\mathrm{P}_{m}$-orbit in $\mathrm{Y}_{m}$ but has degree 2 over $\mathrm{Y}_{m}^{\prime}$. This implies our claim. One checks that $\mathrm{Y}_{m}^{\prime}$ has codimension $n-m-r$ in $\mathrm{Y}_{m}$ and that $r\left(\mathrm{Y}_{m}^{\prime}\right)=r+1$.

If $m=n-r-1$ then $\mathrm{Y}_{m}$ is a divisor in $\mathrm{X}$ and hence is Cohen-Macaulay (for $n=2, r=0$ and $m=1$, we recover Example 1 in Section 1.) But if $m \leq n-r-2$ then $Y_{m}$ has depth 1 along $Y_{m}^{\prime}$ by Serre's criterion, see [11] 18.3. In particular, $\mathrm{Y}_{m}$ is not Cohen-Macaulay.

Let $\alpha_{1}, \ldots, \alpha_{n}$ be the simple roots of $\mathrm{G}$. Then $\mathrm{P}_{\alpha_{m}} \mathrm{Y}_{m}=\mathrm{Y}_{m+1}$, and $\alpha_{m}$ is the unique simple root raising $\mathrm{Y}_{m}$. The corresponding edge in $\Gamma(\mathrm{X})$ is simple, except for $m=n-r-1$. Thus, $\mathrm{Y}_{m}$ is the source of a unique oriented path with target $\mathrm{X}$, and the top edge of this path is double. In particular, $\mathrm{Y}_{m}$ is not multiplicity-free.

Such examples of bad singularities do not occur for multiplicity-free orbit closures:

Theorem 5. Let $\mathrm{X}$ be a spherical variety and let $\mathrm{Y}$ be a multiplicity-free B orbit closure. Then the singularities of $\mathrm{Y}$ are rational. If in addition $\mathrm{X}$ is regular, then for any $\mathrm{G}$-orbit closure $\mathrm{X}^{\prime}$ in $\mathrm{X}$, the scheme-theoretical intersection $\mathrm{Y} \cap \mathrm{X}^{\prime}$ is reduced. Moreover, the local equations of the boundary divisors containing $\mathrm{X}^{\prime}$ at any point of $\mathrm{Y} \cap \mathrm{X}^{\prime}$ are a regular sequence in the local ring of $\mathrm{Y}$ at that point.

Proof. The main point is rationality of singularities, proved by induction on the codimension of $\mathrm{Y}$. Here is an outline of the argument: choose a resolution of singularities $\varphi: \mathrm{Z} \rightarrow \mathrm{Y}$ which is equivariant for the normalizer $\mathrm{N}_{\mathrm{G}}(\mathrm{Y})$, a standard parabolic subgroup of $\mathrm{G}$. This yields $\mathrm{N}_{\mathrm{G}}(\mathrm{Y})$-linearized coherent sheaves on $\mathrm{Y}$ :

$$
\mathcal{C}_{0}=\left(\varphi_{*} \mathcal{O}_{\mathrm{Z}}\right) / \mathcal{O}_{\mathrm{Y}} \quad \text { and } \quad \mathcal{C}_{n}=\mathrm{R}^{n} \varphi_{*} \mathcal{O}_{\mathrm{Z}} \text { for } n \geq 1
$$

For every standard parabolic subgroup $\mathrm{P}$, we can induce these sheaves to $\mathrm{P}$ linearized coherent sheaves $\mathrm{P} \times{ }^{\mathrm{B}} \mathcal{C}_{n}$ on $\mathrm{P} \times{ }^{\mathrm{B}} \mathrm{Y}$. Choose for $\mathrm{P}$ a minimal parabolic subgroup raising $\mathrm{Y}$ and consider the natural map $\pi: \mathrm{P} \times{ }^{\mathrm{B}} \mathrm{Y} \rightarrow \mathrm{PY}$, then $\pi$ is birational, and the singularities of $\mathrm{PY}$ are rational by the induction assumption. Using this, we show that $\pi_{*}\left(\mathrm{P} \times{ }^{\mathrm{B}} \mathcal{C}_{n}\right)=0$ for all $n$, and we deduce that the sheaves $\mathcal{C}_{n}$ are trivial.

We begin the detailed argument by fixing notation. Let $\mathrm{P}$ be a minimal parabolic subgroup that raises $\mathrm{Y}$. Let

$$
f: \mathrm{P} \times{ }^{\mathrm{B}} \mathrm{Y} \rightarrow \mathrm{P} / \mathrm{B} \cong \mathbb{P}^{1}
$$

be the locally trivial fiber bundle with fiber the $\mathrm{B}$-variety $\mathrm{Y}$, and let

$$
\pi: \mathrm{P} \times{ }^{\mathrm{B}} \mathrm{Y} \rightarrow \mathrm{PY}
$$


be the natural morphism, then $\pi$ is birational and $\mathrm{P}$-equivariant. The $\mathrm{B}$ equivariant resolution $\varphi: \mathrm{Z} \rightarrow \mathrm{Y}$ induces a $\mathrm{P}$-equivariant resolution

$$
\rho: \mathrm{P} \times{ }^{\mathrm{B}} \mathrm{Z} \rightarrow \mathrm{P} \times{ }^{\mathrm{B}} \mathrm{Y} .
$$

Composing $\rho$ with $\pi$, we obtain a $\mathrm{P}$-equivariant resolution

$$
\tilde{\pi}: \mathrm{P} \times{ }^{\mathrm{B}} \mathrm{Z} \rightarrow \mathrm{PY}
$$

We have an exact sequence

$$
0 \rightarrow \mathcal{O}_{\mathrm{Y}} \rightarrow \varphi_{*} \mathcal{O}_{\mathrm{Z}} \rightarrow \mathcal{C}_{0} \rightarrow 0
$$

of $\mathrm{N}_{\mathrm{G}}(\mathrm{Y})$-linearized coherent sheaves on $\mathrm{Y}$. Moreover, every B-linearized (coherent) sheaf $\mathcal{F}$ on $\mathrm{Y}$ corresponds to a $\mathrm{P}$-linearized sheaf $\mathrm{P} \times{ }^{\mathrm{B}} \mathcal{F}$ on $\mathrm{P} \times{ }^{\mathrm{B}} \mathrm{Y}$, uniquely defined by imposing that the restriction of $\mathrm{P} \times{ }^{\mathrm{B}} \mathcal{F}$ to $\mathrm{B} \times{ }^{\mathrm{B}} \mathrm{Y} \cong \mathrm{Y}$ is $\mathcal{F}$; and $\mathrm{P} \times{ }^{\mathrm{B}} \mathcal{O}_{\mathrm{Y}}=\mathcal{O}_{\mathrm{P} \times{ }^{\mathrm{B}} \mathrm{Y}}$, whereas $\mathrm{P} \times{ }^{\mathrm{B}} \varphi_{*} \mathcal{O}_{\mathrm{Z}}=\rho_{*} \mathcal{O}_{\mathrm{P} \times{ }^{\mathrm{B}} \mathrm{Z}}$. Thus, we have an exact sequence of $\mathrm{P}$-linearized sheaves on $\mathrm{P} \times{ }^{\mathrm{B}} \mathrm{Y}$ :

$$
0 \rightarrow \mathcal{O}_{\mathrm{P} \times{ }^{\mathrm{B} Y}} \rightarrow \rho_{*} \mathcal{O}_{\mathrm{P} \times{ }^{\mathrm{B} Z}} \rightarrow \mathrm{P} \times{ }^{\mathrm{B}} \mathcal{C}_{0} \rightarrow 0
$$

Applying $\pi_{*}$, we obtain an exact sequence of $\mathrm{P}$-linearized sheaves on PY :

$$
0 \rightarrow \pi_{*} \mathcal{O}_{\mathrm{P} \times{ }^{\mathrm{B} Y}} \rightarrow \tilde{\pi}_{*} \mathcal{O}_{\mathrm{P} \times{ }^{\mathrm{B} Z}} \rightarrow \pi_{*}\left(\mathrm{P} \times{ }^{\mathrm{B}} \mathcal{C}_{0}\right) \rightarrow \mathrm{R}^{1} \pi_{*} \mathcal{O}_{\mathrm{P} \times{ }^{\mathrm{B} Y}}
$$

Moreover, $\pi_{*} \mathcal{O}_{\mathrm{P} \times{ }^{\mathrm{B} Y}}=\tilde{\pi}_{*} \mathcal{O}_{\mathrm{P} \times{ }^{\mathrm{B} Z}}=\mathcal{O}_{\mathrm{PY}}$, since $\mathrm{PY}$ is normal. Thus, $\pi_{*}\left(\mathrm{P} \times{ }^{\mathrm{B}} \mathcal{C}_{0}\right)$ injects into $\mathrm{R}^{1} \pi_{*} \mathcal{O}_{\mathrm{P} \times{ }^{\mathrm{B}} \mathrm{Y}}$.

We claim that $\mathrm{R}^{1} \pi_{*} \mathcal{O}_{\mathrm{P} \times{ }^{\mathrm{B}} \mathrm{Y}}=0$. For this, we factor $\pi$ as the closed immersion

$$
i=(f, \pi): \mathrm{P} \times{ }^{\mathrm{B}} \mathrm{Y} \rightarrow \mathrm{P} / \mathrm{B} \times \mathrm{PY}
$$

followed by the projection

$$
p: \mathrm{P} / \mathrm{B} \times \mathrm{PY} \rightarrow \mathrm{P} / \mathrm{B}
$$

with fibers $\mathbb{P}^{1}$. Then $\mathrm{R}^{1} \pi_{*} \mathcal{O}_{\mathrm{P} \times{ }^{\mathrm{B} Y}}=\mathrm{R}^{1} p_{*}\left(i_{*} \mathcal{O}_{\mathrm{P} \times{ }^{\mathrm{B} Y}}\right)$. Moreover, we have an exact sequence

$$
0 \rightarrow \mathcal{I} \rightarrow \mathcal{O}_{\mathrm{P} / \mathrm{B} \times \mathrm{PY}} \rightarrow i_{*} \mathcal{O}_{\mathrm{P} \times{ }^{\mathrm{B} Y}} \rightarrow 0
$$

where $\mathcal{I}$ is the ideal sheaf of $\mathrm{P} \times{ }^{\mathrm{B}} \mathrm{Y}$. This yields an exact sequence

$$
\mathrm{R}^{1} p_{*} \mathcal{O}_{\mathrm{P} / \mathrm{B} \times \mathrm{PY}} \rightarrow \mathrm{R}^{1} p_{*}\left(i_{*} \mathcal{O}_{\mathrm{P} \times{ }^{\mathrm{B}} \mathrm{Y}}\right) \rightarrow \mathrm{R}^{2} p_{*} \mathcal{I}
$$

But $\mathrm{R}^{2} p_{*} \mathcal{I}=0$, as $p$ is proper with fibers of dimension 1 ; and $\mathrm{R}^{1} p_{*} \mathcal{O}_{\mathrm{P} / \mathrm{B} \times \mathrm{PY}}=$ 0 , as $\mathrm{H}^{1}\left(\mathbb{P}^{1}, \mathcal{O}_{\mathbb{P}^{1}}\right)=0$. This proves our claim; it results that $\pi_{*}\left(\mathrm{P} \times{ }^{\mathrm{B}} \mathcal{C}_{0}\right)=0$.

Next we claim that $\pi_{*}\left(\mathrm{P} \times{ }^{\mathrm{B}} \mathcal{C}_{n}\right)=0$ for every $n \geq 1$, where $\mathcal{C}_{n}=\mathrm{R}^{n} \varphi_{*} \mathcal{O}_{\mathrm{Z}}$. For this, we use the Leray spectral sequence associated with $\tilde{\pi}=\pi \circ \rho$. Since $\tilde{\pi}$ is a resolution of $\mathrm{PY}$, the induction assumption yields that $\mathrm{R}^{q} \tilde{\pi}_{*} \mathcal{O}_{\mathrm{P} \times{ }^{\mathrm{B}} \mathrm{Z}}=0$ for all $q \geq 1$. On the other hand, $\mathrm{R}^{p} \pi_{*} \mathcal{F}=0$ for any $p \geq 2$ and for any coherent sheaf $\mathcal{F}$ on $\mathrm{P} \times{ }^{\mathrm{B}} \mathrm{Y}$, as $\pi$ is proper with fibers of dimension $\leq 1$. It follows that the spectral sequence

$$
\mathrm{R}^{p} \pi_{*}\left(\mathrm{R}^{q} \rho_{*} \mathcal{O}_{\mathrm{P} \times{ }^{\mathrm{B}} \mathrm{Z}}\right) \Rightarrow \mathrm{R}^{p+q} \tilde{\pi}_{*} \mathcal{O}_{\mathrm{P} \times{ }^{\mathrm{B}} \mathrm{Z}}
$$


degenerates at $\mathrm{E}_{2}$ : then $\pi_{*}\left(\mathrm{R}^{n} \rho_{*} \mathcal{O}_{\mathrm{P} \times \mathrm{B} Z}\right)$ is a quotient of $\mathrm{R}^{n} \tilde{\pi}_{*} \mathcal{O}_{\mathrm{P} \times \mathrm{B}}$, and hence is trivial. Moreover, $\left.\mathrm{R}^{n} \rho_{*} \mathcal{O}_{\mathrm{P} \times \mathrm{B}}\right)=\mathrm{P} \times{ }^{\mathrm{B}}\left(\mathrm{R}^{n} \varphi_{*} \mathcal{O}_{\mathrm{Z}}\right)=\mathrm{P} \times{ }^{\mathrm{B}} \mathcal{C}_{n}$, since $\rho: \mathrm{P} \times{ }^{\mathrm{B}} \mathrm{Z} \rightarrow \mathrm{P} \times{ }^{\mathrm{B}} \mathrm{Y}$ is induced from $\varphi: \mathrm{Z} \rightarrow \mathrm{Y}$. This proves the claim.

We now need the following

Lemma 8. Let $\mathcal{F}$ be a coherent $\mathrm{N}_{\mathrm{G}}(\mathrm{Y})$-linearized sheaf on $\mathrm{Y}$ such that $\pi_{*}\left(\mathrm{P} \times{ }^{\mathrm{B}}\right.$ $\mathcal{F})$ is trivial for any minimal parabolic subgroup $\mathrm{P}$ that raises $\mathrm{Y}$. Then the support of $\mathcal{F}$ is $\mathrm{G}$-stable.

Proof. Let $\mathrm{Y}^{\prime}$ be an irreducible component of the support of $\mathcal{F}$, with ideal sheaf $\mathcal{I}_{\mathrm{Y}^{\prime}}$. Define a subsheaf $\mathcal{F}^{\prime}$ of $\mathcal{F}$ by

$$
\mathcal{F}^{\prime}(\mathrm{V})=\left\{s \in \mathcal{F}(\mathrm{V}) \mid \mathcal{I}_{\mathrm{Y}^{\prime}}(\mathrm{V}) s=0\right\}
$$

for every open subset $\mathrm{V}$ of $\mathrm{Y}$. Observe that $\mathcal{F}^{\prime}$ is a coherent sheaf of $\mathcal{O}_{\mathrm{Y}^{\prime}}$ modules, with support precisely $\mathrm{Y}^{\prime}$ (indeed, if $\mathrm{V}$ is affine, then the ideal $\mathcal{I}_{\mathrm{Y}^{\prime}}(\mathrm{V})$ is a minimal prime of the support of $\mathcal{F}(\mathrm{V})$; thus, this ideal is an associated prime of $\mathcal{F}(\mathrm{V})$.) Note that $\mathrm{N}_{\mathrm{G}}(\mathrm{Y})$ stabilizes $\mathrm{Y}^{\prime}$ and acts on $\mathcal{F}^{\prime}$. Moreover, $\pi_{*}\left(\mathrm{P} \times{ }^{\mathrm{B}} \mathcal{F}^{\prime}\right)$ is trivial, since $\pi_{*}\left(\mathrm{P} \times{ }^{\mathrm{B}} \mathcal{F}\right)$ is.

Assume that $\mathrm{Y}^{\prime}$ is not $\mathrm{G}$-stable and let $\mathrm{P}$ be a minimal parabolic subgroup raising $\mathrm{Y}^{\prime}$; then $\mathrm{P}$ raises $\mathrm{Y}$, for $\mathrm{Y}^{\prime}$ is stable under $\mathrm{N}_{\mathrm{G}}(\mathrm{Y})$. Let $\pi^{\prime}: \mathrm{P}^{\mathrm{B}} \mathrm{Y}^{\prime} \rightarrow$ $\mathrm{PY}^{\prime}$ be the restriction of $\pi$ to $\mathrm{P} \times{ }^{\mathrm{B}} \mathrm{Y}^{\prime}$. Then $\pi^{\prime}$ is generically finite (as $\mathrm{P}$ raises $\mathrm{Y}^{\prime}$ ), and the support of $\mathrm{P} \times{ }^{\mathrm{B}} \mathcal{F}^{\prime}$ is $\mathrm{P} \times{ }^{\mathrm{B}} \mathrm{Y}^{\prime}$ (as the support of $\mathcal{F}^{\prime}$ is $\left.\mathrm{Y}^{\prime}\right)$. Thus, the support of $\pi_{*}^{\prime}\left(\mathrm{P} \times{ }^{\mathrm{B}} \mathcal{F}^{\prime}\right)$ is $\mathrm{PY}^{\prime}$. But this contradicts the fact that $\pi_{*}^{\prime}\left(\mathrm{P} \times{ }^{\mathrm{B}} \mathcal{F}^{\prime}\right)=\pi_{*}\left(\mathrm{P} \times{ }^{\mathrm{B}} \mathcal{F}^{\prime}\right)$ is trivial.

Thus, the support of each $\mathcal{C}_{n}$ is $\mathrm{G}$-stable. On the other hand, we may assume that $\mathrm{Y}$ contains no G-orbit (to see this, replace $\mathrm{G}$ (resp. X) by $\tilde{\mathrm{G}}=\mathrm{G} \times \mathrm{PGL}(2)$ (resp. $\tilde{\mathrm{X}}=\mathrm{X} \times \mathrm{P}^{1}$ with the natural $\tilde{\mathrm{G}}$-action). Then $\tilde{\mathrm{X}}$ is a spherical $\tilde{\mathrm{G}}$-variety, and $\tilde{Y}=\mathrm{Y} \times\{\infty\} \simeq \mathrm{Y}$ is multiplicity-free and contains no $\tilde{\mathrm{G}}$-orbit). It then follows that each $\mathcal{C}_{n}$ is trivial. We have proved that the singularities of $\mathrm{Y}$ are rational.

For the remaining assertions, let $\mathrm{D}_{1}, \ldots, \mathrm{D}_{r}$ be the boundary divisors containing $\mathrm{X}^{\prime}$, then the local equations of $\mathrm{D}_{1}, \ldots, \mathrm{D}_{r}$ at any point $x \in \mathrm{X}^{\prime}$ are a regular sequence in $\mathcal{O}_{\mathrm{X}, x}$. Moreover, the scheme-theoretical intersection $\mathrm{Y} \cap \mathrm{X}^{\prime}$ is equidimensional of codimension $r$ and generically reduced. Since $\mathrm{Y}$ is CohenMacaulay, then $\mathrm{Y} \cap \mathrm{X}^{\prime}$ is reduced and the local equations of $\mathrm{D}_{1}, \ldots, \mathrm{D}_{r}$ at any point $y \in \mathrm{Y} \cap \mathrm{X}^{\prime}$ are a regular sequence in $\mathcal{O}_{\mathrm{Y}, y}$.

We now apply these results to $\mathrm{H}$-orbit closures in the flag variety of $\mathrm{G}$. For this, we recall a construction from [6] 1.5. Let $\mathrm{V}$ be a $\mathrm{H}$-orbit closure in $\mathrm{G} / \mathrm{B}$ and let $\hat{\mathrm{V}}$ be the corresponding $\mathrm{B}$-orbit closure in $\mathrm{G} / \mathrm{H}$. Let $\mathrm{Y}$ be the closure of $\hat{\mathrm{V}}$ in a regular completion $\mathrm{X}$, then $\mathrm{Y} \in \mathcal{B}(\mathrm{G} / \mathrm{H}, \mathrm{X})$. Consider the natural 
morphism

$$
\pi: \mathrm{G} \times{ }^{\mathrm{B}} \mathrm{Y} \rightarrow \mathrm{X}
$$

and the projection

$$
f: \mathrm{G} \times{ }^{\mathrm{B}} \mathrm{Y} \rightarrow \mathrm{G} / \mathrm{B} .
$$

The fibers of $\pi$ identify to closed subschemes of $\mathrm{G} / \mathrm{B}$ via $f_{*}$. Let $x$ be the image in $\mathrm{X}$ of the base point of $\mathrm{G} / \mathrm{H}$, then $\pi^{-1}(x)$ identifies to $\mathrm{V}$. On the other hand, let $\mathrm{Z}$ be a closed $\mathrm{G}$-orbit in $\mathrm{X}$ with $\mathrm{B}$-fixed point $z$, then the set $f\left(\pi^{-1}(z)\right)$ equals

$$
\mathrm{V}_{0}=\bigcup_{w \in \mathrm{W}(\mathrm{Y})} \overline{\mathrm{B} w_{0} w \mathrm{~B}} / \mathrm{B}
$$

where $w_{0}$ denotes the longest element of $\mathrm{W}$. Moreover, we have in the integral cohomology ring of $\mathrm{G} / \mathrm{B}$ :

$$
[\mathrm{V}]=\sum_{w \in \mathrm{W}(\mathrm{Y})} d(\mathrm{Y}, w)\left[\overline{\mathrm{B} w_{0} w \mathrm{~B}} / \mathrm{B}\right]
$$

Now Theorem 2 and Proposition 5 imply the following

Corollary 7. With notation as above, $\mathrm{V}_{0}$ is connected in codimension 1 . If moreover $\mathrm{G}$ is simply-laced, then $[\mathrm{V}]=2^{\ell_{\mathrm{N}}(\gamma)}\left[\mathrm{V}_{0}\right]$ where $\gamma$ is any oriented path in $\Gamma(\mathrm{X})$ joining $\mathrm{Y}$ to $\mathrm{X}$.

We shall call $\mathrm{V}$ multiplicity-free if $\mathrm{Y}$ is. Equivalently, the cohomology class of $\mathrm{V}$ decomposes as a sum of Schubert classes with coefficients 0 or 1.

Note that any multiplicity-free $\mathrm{H}$-orbit closure $\mathrm{V}$ is irreducible, even if $\mathrm{H}$ is not connected. Indeed, $\mathrm{H}$ acts transitively on the set of all irreducible components of $\mathrm{V}$, so that any two such components have the same cohomology class; but the class of $\mathrm{V}$ is indivisible in the integral cohomology of G/B .

Theorem 6. With notation as above, if $\mathrm{V}$ is multiplicity-free then its singularities are rational, the morphism $\pi$ is flat, and its fibers are reduced. As a consequence, the fibers of $\pi$ realize a degeneration of $\mathrm{V}$ to the reduced subscheme $\mathrm{V}_{0}$ of $\mathrm{G} / \mathrm{B}$.

Proof. Note that the singularities of $\mathrm{Y}$ are rational by Theorem 5; thus, the same holds for $\hat{\mathrm{V}}=\mathrm{Y} \cap \mathrm{G} / \mathrm{H}$. Let $\varphi: \mathrm{Z} \rightarrow \hat{\mathrm{V}}$ be a resolution of singularities; consider the quotient map $q_{H}: \mathrm{G} \rightarrow \mathrm{G} / \mathrm{H}$, the preimage $\mathrm{V}^{\prime}=q_{H}^{-1}(\hat{\mathrm{V}})$ in $\mathrm{G}$, and the fiber product $\mathrm{Z}^{\prime}=\mathrm{Z} \times{ }_{\hat{\mathrm{V}}} \mathrm{V}^{\prime}$. Then $\mathrm{Z}^{\prime}$ is smooth, since $\mathrm{Z}$ and $q_{H}$ are; the projection $\varphi^{\prime}: \mathrm{Z}^{\prime} \rightarrow \mathrm{V}^{\prime}$ is proper and birational, since $\varphi$ is; and $\mathrm{R}^{n} \varphi_{*}^{\prime} \mathcal{O}_{\mathrm{Z}^{\prime}}=0$ for $n \geq 1$, since cohomology commutes with flat base extension. Therefore the singularities of $\mathrm{V}^{\prime}$ are rational. Now $\mathrm{V}^{\prime}=q_{\mathrm{B}}^{-1}(\mathrm{~V})$ and $q_{\mathrm{B}}$ is a locally trivial fibration, so that the singularities of $\mathrm{V}$ are rational as well. 
For the second assertion, we identify $\mathrm{Y}$ to its image $\mathrm{B} \times{ }^{\mathrm{B}} \mathrm{Y}$ in $\mathrm{G} \times{ }^{\mathrm{B}} \mathrm{Y}$. Since $\pi$ is $\mathrm{G}$-equivariant, it is enough to check the statement at $y \in \mathrm{Y}$. Let $\mathrm{D}_{1}, \ldots, \mathrm{D}_{r}$ be the boundary divisors containing $y$, with local equations $f_{1}, \ldots, f_{r}$ in $\mathcal{O}_{\mathrm{X}, y}$. It follows from Theorem 5 that the pull-backs $\pi^{*} f_{1}, \ldots, \pi^{*} f_{r}$ are a regular sequence in $\mathcal{O}_{\mathrm{G} \times{ }^{\mathrm{B} Y}, y}$ and generate the ideal of $\pi^{-1}(\mathrm{G} y)$. Moreover, the restriction of $\pi$ to $\pi^{-1}(\mathrm{G} y)$ is flat with reduced fibers, as $\pi$ is $\mathrm{G}$-equivariant. Now we conclude by a local flatness criterion, see [11] Corollary 6.9.

A direct consequence is the following

Corollary 8. Consider a a multiplicity-free $\mathrm{H}$-orbit closure $\mathrm{V}$ in $\mathrm{G} / \mathrm{B}$ and an effective line bundle $\mathrm{L}$ on $\mathrm{G} / \mathrm{B}$. Then the restriction map $H^{0}(\mathrm{G} / \mathrm{B}, \mathrm{L}) \rightarrow$ $H^{0}(\mathrm{~V}, \mathrm{~L})$ is surjective, and $H^{n}(\mathrm{~V}, \mathrm{~L})=0$ for all $n \geq 1$.

Indeed, this holds with $\mathrm{V}$ replaced by $\mathrm{V}_{0}$, a union of Schubert varieties (see [19].) The result follows by semicontinuity of cohomology in a flat family.

We now obtain a partial converse to Corollary 8:

Proposition 10. Let $\mathrm{V}$ be a $\mathrm{H}$-orbit closure in $\mathrm{G} / \mathrm{B}$ with corresponding $\mathrm{B}$ orbit closure $\mathrm{Y}$ in $\mathrm{G} / \mathrm{H}$. If $\mathrm{Y}$ is the source of a double edge of $\Gamma(\mathrm{G} / \mathrm{H})$, then there exists an effective line bundle $\mathrm{L}$ on $\mathrm{G} / \mathrm{B}$ such that the restriction $H^{0}(\mathrm{G} / \mathrm{B}, \mathrm{L}) \rightarrow H^{0}(\mathrm{~V}, \mathrm{~L})$ is not surjective.

Proof. Let $\alpha$ be the label of a double edge with source Y . Denote by $p: \mathrm{G} / \mathrm{B} \rightarrow$ $\mathrm{G} / \mathrm{P}_{\alpha}$ the natural map and by $p_{\mathrm{V}}: \mathrm{V} \rightarrow \pi(\mathrm{V})$ its restriction to $\mathrm{V}$, then $p$ is a projective line bundle and $p_{\mathrm{V}}$ is generically finite of degree 2. Choose an ample line bundle $\mathrm{L}$ on $\mathrm{G} / \mathrm{P}_{\alpha}$, then $p^{*} \mathrm{~L}$ is an effective line bundle on $\mathrm{G} / \mathrm{B}$. Now it suffices to check the following claim: the restriction map

$$
r_{n}: H^{0}\left(p^{-1} p(\mathrm{~V}), p^{*}\left(\mathrm{~L}^{\otimes n}\right)\right) \rightarrow H^{0}\left(\mathrm{~V}, p^{*}\left(\mathrm{~L}^{\otimes n}\right)\right)
$$

is not surjective for large $n$.

For this, note that

$$
\begin{aligned}
H^{0}\left(p^{-1} p(\mathrm{~V}), p^{*}\left(\mathrm{~L}^{\otimes n}\right)\right) & =H^{0}\left(p(\mathrm{~V}), \mathrm{L}^{\otimes n}\right) \\
H^{0}\left(\mathrm{~V}, p^{*}\left(\mathrm{~L}^{\otimes n}\right)\right) & =H^{0}\left(p(\mathrm{~V}), \mathrm{L}^{\otimes n} \otimes p_{\mathrm{V}_{*}} \mathcal{O}_{\mathrm{V}}\right)
\end{aligned}
$$

by the projection formula. Thus, $r_{n}$ identifies with the map

$$
H^{0}\left(p(\mathrm{~V}), \mathrm{L}^{\otimes n}\right) \rightarrow H^{0}\left(p(\mathrm{~V}), \mathrm{L}^{\otimes n} \otimes p_{\mathrm{V}_{*}} \mathcal{O}_{\mathrm{V}}\right)
$$

defined by the inclusion of $\mathcal{O}_{p(\mathrm{~V})}$ into $p_{\mathrm{V}_{*}} \mathcal{O}_{\mathrm{V}}$. Since $p_{\mathrm{V}}$ has degree 2 , the quotient $\mathcal{F}=p_{\mathrm{V}_{*}} \mathcal{O}_{\mathrm{V}} / \mathcal{O}_{p(\mathrm{~V})}$ has rank 1 as a sheaf of $\mathcal{O}_{p(\mathrm{~V})}$-modules. Moreover, since $\mathrm{L}$ is ample, the cokernel of $r_{n}$ is isomorphic to $H^{0}\left(p(\mathrm{~V}), \mathcal{F} \otimes \mathrm{L}^{\otimes n}\right)$ for large $n$. This implies the claim. 


\section{Acknowledgements}

A first version of this paper is available at math.AG/9908094. I thank Peter Littelmann, Laurent Manivel, Olivier Mathieu, Stéphane Pin, Patrick Polo, Tonny Springer and especially Thierry Vust for useful comments, discussions or e-mail exchanges.

\section{References}

[1] D. Barbasch, S. Evens, K -orbits on Grassmannians and a PRV conjecture for real groups, J. Algebra 167 (1994), 258-283.

[2] E. Bifet, C. de Concini, C. Procesi, Cohomology of regular embeddings, Adv. Math. 82 (1990), 1-34.

[3] F. Bien, M. Brion, Automorphisms and local rigidity of regular varieties, Compositio Math. 104 (1996), 1-26.

[4] M. Brion, A. Helminck, On orbit closures of symmetric subgroups in flag varieties, Canadian J. Math. 52 (2000), 265-292.

[5] M. Brion, S. P. Inamdar, Frobenius splitting and spherical varieties, in: Algebraic groups and their generalizations, Proc. Symp. Pure Math. 56, Part 1, AMS, Providence 1994, 207-218.

[6] M. Brion, The behaviour at infinity of the Bruhat decomposition, Comment. Math. Helv. 73 (1998), 137-174

[7] M. Brion, Rational smoothness and fixed points of torus actions, Transformation Groups 4 (1999), 127-156.

[8] M. Brion, P. Polo, Large Schubert varieties, Representation Theory 4 (2000), 97-126.

[9] M. Demazure, Automorphismes et déformations des variétés de Borel, Invent. math. 39 (1977), 179-186.

[10] V. V. Deodhar, Some characterizations of Bruhat ordering on a Coxeter group and determination of the relative Mobius function, Invent. math. 39 (1977), 187-198.

[11] D. Eisensud, Commutative algebra with a view towards algebraic geometry, SpringerVerlag 1994.

[12] W. Fulton, Intersection theory, Springer-Verlag 1984.

[13] J. E. Humphreys, Reflection groups and Coxeter groups, Cambridge University Press 1990.

[14] G. Kempf, F. Knudsen, D. Mumford, B. Saint-Donat, Toroidal embeddings I, Lecture Notes in Math. 339, Springer-Verlag 1973.

[15] F. Knop, The Luna-Vust theory of spherical embeddings. In: Proceedings of the Hyderabad conference on algebraic groups, Manoj Prakashan, Madras 1991, 225-248.

[16] F. Knop, A Harish-Chandra homomorphism for reductive group actions, Ann. of Math. 140 (1994), 253-288.

[17] F. Knop, The asymptotic behavior of invariant collective motion, Invent. math. 116 (1994), 309-328.

[18] F. Knop, On the set of orbits for a Borel subgroup, Comment. Math. Helv. 70 (1995), 285-309.

[19] V. B. Mehta, A. Ramanathan, Frobenius splitting and cohomology vanishing for Schubert varieties, Ann. of Math. 122 (1985), 27-40.

[20] R. W. Richardson, T. A. Springer, The Bruhat order on symmetric varieties, Geom. Dedicata 35 (1990), 389-436.

[21] R. W. Richardson, T. A. Springer, Combinatorics and geometry of K -orbits on the flag manifold. In: Linear algebraic groups and their representations (R. Elman, M. Schacher, V. Varadarajan eds.), Contemp. Math. 153, AMS, Providence 1993, 109-142. 
[22] C. S. Seshadri, Line bundles on Schubert varieties, in: Vector bundles on algebraic varieties, Oxford University Press 1987, 499-528.

[23] T. A. Springer, Schubert varieties and generalizations. In: Representation theories and algebraic geometry (A. Broer ed), Kluwer, Dordrecht 1997, 413-440.

[24] T. A. Springer, An invariant of K -orbits in the flag variety, preprint (2000).

[25] T. Vust, Plongements d'espaces symétriques algébriques: une classification, Ann. Scuola Norm. Sup. Pisa 17 (1990), 165-195.

[26] B. Wasserman, Wonderful varieties of rank two, Transformation Groups 1 (1996), 375403.

Michel Brion

Université de Grenoble I

Département de Mathématiques

Institute Fourier

UMR 5582 du CNRS

F-38402 Saint-Martin d'Hères Cedex

France

e-mail: Michel.Brion@ujf-grenoble.fr

(Received: April 17, 2000) 